\title{
Optimal Target Criteria for Stabilization Policy*
}

\author{
Marc P. Giannoni \\ Michael Woodford \\ FRB New York ${ }^{\dagger}$ \\ Columbia University
}

November 25, 2016

\begin{abstract}
This paper considers a general class of nonlinear rational-expectations models in which policymakers seek to maximize an objective function that may be household expected utility. We show how to derive a target criterion that is: (i) consistent with the model's structural equations, (ii) strong enough to imply a unique equilibrium, and (iii) optimal, in the sense that a commitment to adjust the policy instrument at all dates so as to satisfy the target criterion maximizes the objective function. The proposed optimal target criterion is a linear equation that must be satisfied by the projected paths of certain economically relevant "target variables." It takes the same form at all times and generally involves only a small number of target variables, regardless of the size and complexity of the model. While the projected path of the economy requires information about the current state, the target criterion itself can be stated without reference to a complete description of the state of the world. We illustrate the application of the method to a nonlinear DSGE model with staggered price-setting, in which the objective of policy is to maximize household expected utility.
\end{abstract}

Keywords: Optimal policy, target criterion, dynamic model, optimal control. JEL codes: C61, C62, E32, E52

\footnotetext{
${ }^{*}$ The views expressed herein are solely those of the authors and do not necessarily reflect those of the Federal Reserve Bank of New York or the Federal Reserve System.

${ }^{\dagger}$ Federal Reserve Bank of New York, Research and Statistics Group, 33 Liberty Street, New York, NY 10045-0001. E-mail: marc.giannoni@ny.frb.org.

${ }^{\ddagger}$ Department of Economics, Columbia University, 420 W. 118th Street, New York, NY $10027 . \quad$ E-mail: michael. woodford@columbia.edu.
}

(C) 2016. This manuscript version is made available under the Elsevier user license http://www.elsevier.com/open-access/userlicense/1.0/ 


\section{Introduction}

Forecast targeting has become an increasingly popular approach, both to the organization of monetary policy deliberations and to communication with the public about monetary policy decisions, at central banks around the world. In this approach, a contemplated forward path for policy is judged correct to the extent that quantitative projections for one or more economic variables, conditional on the contemplated policy, conform to a target criterion. ${ }^{1}$ The present paper considers whether the conditions required for the conduct of policy to maximize welfare can be cast in the form of such a target criterion. ${ }^{2}$ This has previously been shown to be possible in special examples in Svensson and Woodford (2005) and Giannoni and Woodford (2005). These papers showed, for instance, that in the simple linearized New Keynesian model with a quadratic policy objective function that depends on variation in inflation $\pi_{t}$ and the output gap $x_{t}$, the optimal target criterion can be expressed as a simple linear combination of the form:

$$
\pi_{t}+\phi\left(x_{t}-x_{t-1}\right)=0
$$

where $\phi$ is a coefficient that depends on the model's structural parameters. A commitment to setting the policy instrument (e.g., the short-term interest rate) at each date and in each state so as to satisfy this relationship implements the optimal equilibrium.

In this paper, we consider a general class of stabilization problems, in which the set of possible equilibrium evolutions of the economy is determined by a system of structural equations characterizing the behavior and constraints of the private sector. (Our abstract framework need not apply only to monetary policy, ${ }^{3}$ though that is the leading example that motivates our formulation of the problem.)

We show that it is possible quite generally to choose a target criterion with three important properties. First, we seek a target criterion that is consistent with the structural equations. Second, the target criterion should be strong enough to imply a determinate forward path for the economy.

\footnotetext{
${ }^{1}$ For further discussion and examples, see, e.g., Svensson (1997, 2005). Advantages of formulating policy in terms of a target criterion, rather than an explicit instrument rule, are discussed in Svensson (2003, 2011) and Woodford (2011, 2012).

${ }^{2}$ The present exposition offers a simpler expression for the optimal target criterion than in our previous attempt at a general theory, in Giannoni and Woodford (2003a), in addition to allowing a considerably more general class of possible objectives for policy, so that the present approach can be applied to problems, like the one in section 4, where the objective of policy is assumed to be the maximization of household utility.

${ }^{3}$ Benigno and Woodford (2004) show how (joint) optimal monetary and fiscal policy can be implemented by having the monetary and fiscal authorities each responsible for satisfaction of a target criterion.
} 
Thus we must verify that there exists an evolution that satisfies the target criterion, looking forward from any possible situation that may have been reached, and also that the evolution consistent with the target criterion is unique. Third, we seek a target criterion such that the state-contingent evolution determined by the criterion is optimal, in the sense of maximizing an ex ante expected welfare criterion.

It might be thought that a sufficient solution to this problem would simply be to compute the optimal state-contingent evolution of all endogenous variables, under an optimal commitment chosen at some initial date $t_{0}$, and to refer to the solution to this problem at any later date $t$ to determine what forward path for policy from date $t$ onward is consistent with the optimal equilibrium. In practice, however, such a once-and-for-all description of the optimal evolution under all possible situations that can ever arise will be impractical, even for decision-making within an institution like a central bank, let alone for communication with the public about the basis for policy decisions.

Policymakers have a great deal of information about the specific situation that has arisen, once it arises, without having any corresponding ability to list all of the situations that may arise very far in advance. The target criterion that we seek is accordingly one that allows a forward path for policy to be selected at each date, looking forward from the particular situation that has arisen at that date, without any reference to all of the paths that the economy might have taken but has not.

Moreover, the target criterion that we propose can be stated without a complete description of the state of the world in which it is to be applied. The target criterion is a linear equation that must be satisfied by the projected paths of certain "target variables"; while a determination of the forward path of policy required for the economy's evolution to satisfy the criterion will involve finegrained information about the current state, the target criterion itself (which takes the same form at all times and involves only a small number of target variables) can be stated without reference to such a complete description of the state.

The methods that we use to derive the optimal target criterion are related to methods used in the literature on (discrete-time, stochastic) optimal control, but our approach differs in important respects. In the standard theory of optimal control (e.g., Anderson and Moore, 1979; Hansen and Sargent, 2013), a "policy equation" is derived that specifies a vector of policy instruments (or "controls") as a function of a state vector that includes a complete description of both current disturbances and all information available at a given point in time about future disturbances. When 
the theory is extended to deal with optimal policy choice subject to forward-looking constraints, the policy equation specifies the instruments as functions not only of the state vector but also of a vector of Lagrange multipliers measuring the value of inducing alternative expectations about conditions at an earlier date. ${ }^{4}$

The characterization we that seek is instead independent of the choice of the instruments of policy - for example, the optimal target criterion for monetary stabilization policy takes the same form, regardless of whether the central bank is expected to use a short-term nominal interest rate as its policy instrument, or the money supply, in a model where one of the structural relations can be used to determine the money supply required to achieve a given equilibrium interest rate and vice versa - and instead specifies only a relationship that policy should aim to bring about among "target variables" that can be influenced by policy. Moreover, we seek a relationship that can be stated without reference to the complete state vector (which in practical applications must be a very large state vector, if one that can even be written down), and that involves only economically relevant "target variables" — variables for which quantitative projections have meaning independent of the particular model of the economy that may be used to produce the projections - rather than abstract concepts such as the Lagrange multipliers associated with a particular optimization problem. We also seek to provide a target criterion that involves the smallest possible set of target variables, and the fewest possible leads and lags, given the structure of the model. ${ }^{5}$

The paper is organized as follows. Section 2 presents the general class of nonlinear optimization problems including both backward-looking and forward-looking constraints with which we are concerned. Section 3 derives a local linear approximation to the optimal equilibrium dynamics in such a problem, and states some important algebraic properties of the coefficients of the equations describing these dynamics. (Here we generalize standard results from the theory of optimal control to a case in which the controls and their effects are not specified.) Section 4 then gives the general form of the optimal target criterion, and establishes that it does indeed determine a (locally) unique equilibrium that coincides with the optimal evolution characterized in section 3 . Section 5 illustrates the application of the method to a nonlinear DSGE model with staggered price-setting. Section 6 concludes.

\footnotetext{
${ }^{4}$ Important early expositions of this approach include Backus and Driffill (1986) and Currie and Levine (1993).

${ }^{5} \mathrm{It}$ is always possible to also propose more complex target criteria that would also implement the optimal responses to shocks; see Giannoni and Woodford (2003a) for further discussion.
} 


\section{A General Nonlinear Framework}

We consider a general nonlinear forward-looking model describing the behavior of the private sector and a general objective function for the policy authority, as in the analysis of Benigno and Woodford (2012). In this section, we derive nonlinear first-order conditions for the policy problem, and use these to define the steady state around which our local analysis of optimal stabilization in response to shocks is conducted.

\subsection{The Model}

Consider the following optimal policy problem. The policy authority seeks to determine which state-contingent evolution of the $m$-dimensional vector of endogenous variables $\left\{y_{t}\right\}$ for $t \geq t_{0}$ will maximize the objective

$$
V_{t_{0}} \equiv E_{t_{0}} \sum_{t=t_{0}}^{\infty} \beta^{t-t_{0}} \pi\left(y_{t}, \xi_{t}\right),
$$

where $0<\beta<1, \pi(y, \xi)$ is a twice continuously differentiable function in all of its arguments, and $\xi_{t}$ is a vector of exogenous disturbances. We assume that $y_{t}$ includes variables determined at date $t$ or possibly before. The evolution of these variables must satisfy the $n$ constraints

$$
\begin{aligned}
F\left(y_{t}, \xi_{t} ; y_{t-1}\right) & =0 \\
E_{t}\left[g\left(y_{t}, \xi_{t} ; y_{t+1}\right)\right] & =0
\end{aligned}
$$

each period. Here $F(\cdot)$ and $g(\cdot)$ are respectively $k$-dimensional and $n$ - $k$-dimensional vector-valued functions, twice continuously differentiable with respect to all of their arguments, where $0 \leq k \leq n$. The vector of exogenous disturbances $\xi_{t}$ is assumed to evolve according to some bounded stochastic process (specified further below).

In order to state certain technical regularity conditions below, it is useful to state condition (2.2) in a more general form when $t=t_{0}$, instead writing

$$
F\left(y_{t_{0}}, \xi_{t_{0}} ; y_{t_{0}-1}\right)=\bar{F}_{t_{0}}
$$

where $\bar{F}_{t_{0}}$ is a vector of $k$ small quantities that may differ from zero. ${ }^{6}$ The model structural relations then consist of equation (2.2) for each period $t>t_{0}$, equation (2.3) for each $t \geq t_{0}$, and equation (2.4), for given initial conditions $y_{t_{0}-1}, \bar{F}_{t_{0}}$, and a given exogenous process $\left\{\xi_{t}\right\}$ for the disturbances.

\footnotetext{
${ }^{6}$ The idea is that we wish to consider perturbations of the initial conditions for the policy problem that do not necessarily correspond to changes in the specified values for $y_{t_{0}-1}$ and $\xi_{t_{0}}$.
} 
We assume that $n<m \leq 2 n$. The first inequality is necessary in order for there to be at least one direction each period in which it is possible for policy to continuously vary equilibrium values of one or more of the endogenous variables; the second (which simplifies the algebraic structure of our problem, ${ }^{7}$ while it is not essential for our methods) implies that the number of independent dimensions of policy is not too large relative to the number of endogenous variables in the model. For example, in many classic treatments of monetary stabilization policy, policy choice is assumed to be one-dimensional (the choice of the money supply, or of one nominal interest rate, each period), so that $m=n+1,{ }^{8}$ and this is the case for which we present the most thorough analysis.

Note that in this formulation of the optimal policy problem, we do not specify the "instruments" of policy, and write a set of structural equations that should completely determine the evolution of the endogenous variables under any given evolution of the instruments. ${ }^{9}$ Instead, equations (2.2)-(2.4) represent the conditions that must be satisfied in equilibrium under any possible policy; the existence of instruments under the control of the policy authority is represented by the incompleteness of the set of equations (2.2)-(2.4) relative to the number of endogenous variables to be determined. (Because $n<m$, the variables $y_{t}$ are surely not uniquely determined by the structural relations, even if some subset of them may be, as discussed in Proposition 4 below.) We do not specify the way in which the available instruments of policy must be adjusted in order to bring about a given state-contingent evolution of the endogenous variables, but we assume that the set of equations (2.2)-(2.4) represents a complete set of constraints, so that any evolution of the variables $y_{t}$ consistent with these equations (and satisfying certain bounds, as discussed further below) can in fact be achieved under some policy.

We proceed in this way in order to make it clear that the optimal target criterion does not depend on the choice of the instrument of policy that is adjusted in order to comply with the target criterion. For example, the instrument of monetary policy might be either a short-term nominal interest rate, or the quantity of base money (to consider two familiar cases). The model structural relations may include an equilibrium relation linking these two variables (the condition required in order for the demand for money at the given interest rate to equal the supply of money), but no equation specifying the central bank's operating target for either variable. The structural relation may be taken to determine the implied nominal interest rate, if the quantity of money is the

\footnotetext{
${ }^{7}$ See discussion following Assumption 2 below.

${ }^{8}$ See, for example, Clarida et al., (1999), Giannoni and Woodford (2003b), or Benigno and Woodford (2005).

${ }^{9}$ This is an important difference between our current approach and the characterization of optimal policy in Giannoni and Woodford (2003a).
} 
instrument (as in traditional textbook accounts); or it may be taken to determine the endogenous quantity of base money, if the interest rate is the central bank's instrument (as in the case of most actual central banks). The optimal target criterion that the central bank should seek to satisfy is the same in either case, and depends only on the set of structural relations (possibly including the coefficients of the money-demand relation).

In addition, we assume that the policymaker considers only paths for the economy consistent with initial pre-commitments of the form

$$
g\left(y_{t_{0}-1}, \xi_{t_{0}-1} ; y_{t_{0}}\right)=\bar{g}_{t_{0}}
$$

where $\bar{g}_{t_{0}}$, which may depend on the exogenous state at date $t_{0}$ and on predetermined variables, is chosen according to a self-consistent rule (as discussed further below). If we were instead to consider the problem of maximizing (2.1) subject only to constraints (2.2)-(2.4) and the initial conditions $y_{t_{0}-1}$ and $\bar{F}_{t_{0}}$, the optimal solution would in general not involve a constant value of $y_{t}$ even in the absence of random disturbances (in the case that $k<n$, so that one or more of the structural equations is forward-looking). As a consequence, the unconstrained optimal (or Ramsey) policy cannot generally be described by a time-invariant policy rule; a target criterion designed to implement this policy would have to be indexed by the time that has elapsed since the date at which the optimization was performed. ${ }^{10}$

Here we consider the simpler problem of characterizing policy that is optimal subject to a particular kind of pre-commitment of the form (2.5). "Self-consistency" of the initial pre-commitment means that $\bar{g}_{t_{0}}$ is determined by a rule which is also satisfied for all $t \geq t_{0}$ under the policy that solves this optimization problem, so that the policy chosen can be viewed as fulfilling a commitment that would optimally have been made at an earlier date. ${ }^{11}$ Formally, we require that $\bar{g}_{t_{0}}$ be determined by a function of the form $\bar{g}_{t_{0}}=\bar{g}\left(\xi_{t_{0}} ; e_{t_{0}-1}\right)$, where $e_{t_{0}-1}$ is some vector of sufficient statistics for the history of the endogenous variables up until period $t_{0}-1,{ }^{12}$ with the property

\footnotetext{
${ }^{10}$ Even more problematic for our purposes, it would not be possible to compute a linear approximation to optimal policy by linearizing around steady-state values of the endogenous variables; it would instead be necessary to linearize around a non-constant path for the variables $y_{t}$, greatly increasing the number of derivatives that would have to be evaluated in order to apply such an approach.

${ }^{11}$ We have elsewhere called this a policy that is "optimal from a timeless perspective." See Woodford (1999, 2003, 2011), Giannoni and Woodford (2003a) and Benigno and Woodford (2012) for further discussion.

${ }^{12}$ This vector may include not only lagged values $y_{t_{0}-j}$, but also lagged forecasts $E_{t_{0}-j} y_{t_{0}-j+k}$, for arbitrary $j, k \geq 1$. See section 4.2 for a precise specification.
} 
that under the optimal policy subject to these pre-commitments,

$$
g\left(y_{t-1}, \xi_{t-1} ; y_{t}\right)=\bar{g}\left(\xi_{t} ; e_{t-1}\right)
$$

in each period $t>t_{0}$ as well.

Choice of an initial pre-commitment of this kind results in a problem for which the solution is a steady state $\left(y_{t}=\bar{y}\right.$ for all $t \geq t_{0}$, for some vector $\left.\bar{y}\right)$ in the absence of random disturbances (i.e., in the case in which $\xi_{t}=\bar{\xi}$ with certainty for all $t$ ), and we can approximate optimal policy in the case of small enough disturbances by linearizing around this steady state. Moreover, the constrainedoptimal policy (hereafter simply called "optimal policy") can be described by a time-invariant rule for the determination of $y_{t}$ as a function of exogenous and pre-determined state variables, and as a consequence it is possible to implement the policy using a time-invariant target criterion.

\subsection{Nonlinear First-Order Conditions for Optimal Policy}

We wish to characterize the state-contingent evolution $\left\{y_{t}\right\}$ for $t \geq t_{0}$ that maximizes (2.1) subject to the constraints (2.2)-(2.5). A Lagrangian for this (exact) nonlinear optimal policy problem is given by

$$
\begin{aligned}
\mathcal{L}_{t_{0}}= & V_{t_{0}}+E_{t_{0}} \sum_{t=t_{0}}^{\infty} \beta^{t-t_{0}}\left[\theta_{t}^{\prime} F\left(y_{t}, \xi_{t} ; y_{t-1}\right)+\Theta_{t}^{\prime} g\left(y_{t}, \xi_{t} ; y_{t+1}\right)\right] \\
& +\beta^{-1} \Theta_{t_{0}-1}^{\prime} g\left(y_{t_{0}-1}, \xi_{t_{0}-1} ; y_{t_{0}}\right) \\
= & E_{t_{0}} \sum_{t=t_{0}}^{\infty} \beta^{t-t_{0}}\left[\pi\left(y_{t}, \xi_{t}\right)+\theta_{t}^{\prime} F\left(y_{t}, \xi_{t} ; y_{t-1}\right)+\beta^{-1} \Theta_{t-1}^{\prime} g\left(y_{t-1}, \xi_{t-1} ; y_{t}\right)\right]
\end{aligned}
$$

where $\theta_{t}$ and $\Theta_{t}$ are vectors of Lagrange multipliers of dimensions $k$ and $n-k$ respectively, associated with the constraints $(2.2)-(2.3)$ for any date $t \geq t_{0},{ }^{13}$ and $\beta^{-1} \Theta_{t_{0}-1}^{\prime}$ is the vector of multipliers associated with the constraints (2.5). Differentiating with respect to $y_{t}$ we obtain the $m$ first-order conditions (FOCs)

$$
\begin{aligned}
D_{1} \pi\left(y_{t}, \xi_{t}\right) & +\theta_{t}^{\prime} D_{1} F\left(y_{t}, \xi_{t} ; y_{t-1}\right)+\beta E_{t}\left[\theta_{t+1}^{\prime} D_{3} F\left(y_{t+1}, \xi_{t+1} ; y_{t}\right)\right] \\
& +E_{t}\left[\Theta_{t}^{\prime} D_{1} g\left(y_{t}, \xi_{t} ; y_{t+1}\right)\right]+\beta^{-1} \Theta_{t-1}^{\prime} D_{3} g\left(y_{t-1}, \xi_{t-1} ; y_{t}\right)=0
\end{aligned}
$$

at each date $t \geq t_{0}$, where $D_{j}$ denotes the vector of partial derivatives with respect to the $j$ th argument of the function considered.

\footnotetext{
${ }^{13}$ To be more precise, $\theta_{t_{0}}$ is the vector of multipliers associated with constraints $(2.4)$.
} 
These FOCs can be written more compactly as

$$
\left(D_{1} \pi\left(y_{t}, \xi_{t}\right)\right)^{\prime}+E_{t}\left[\bar{A}^{\prime}\left(y_{t+1}, y_{t}, \xi_{t+1}, \xi_{t}\right) \varphi_{t+1}\right]-\beta^{-1} \bar{I}^{\prime}\left(y_{t}, y_{t-1}, \xi_{t}, \xi_{t-1}\right) \varphi_{t}=0
$$

where $\varphi_{t+1}$ is an $n$-dimensional vector, and $\bar{A}\left(y_{t+1}, y_{t}, \xi_{t+1}, \xi_{t}\right)$ and $\bar{I}\left(y_{t}, y_{t-1}, \xi_{t}, \xi_{t-1}\right)$ are $(n \times m)$ matrices defined as

$$
\begin{aligned}
\varphi_{t+1} & \equiv\left[\begin{array}{c}
\varphi_{1, t+1} \\
\varphi_{2, t+1}
\end{array}\right] \equiv\left[\begin{array}{c}
\theta_{t+1} \\
\Theta_{t}
\end{array}\right] \\
\bar{A}\left(y_{t+1}, y_{t}, \xi_{t+1}, \xi_{t}\right) & \equiv\left[\begin{array}{c}
\beta D_{3} F\left(y_{t+1}, \xi_{t+1} ; y_{t}\right) \\
D_{1} g\left(y_{t}, \xi_{t} ; y_{t+1}\right)
\end{array}\right] \\
\bar{I}\left(y_{t}, y_{t-1}, \xi_{t}, \xi_{t-1}\right) & \equiv\left[\begin{array}{c}
-\beta D_{1} F\left(y_{t}, \xi_{t} ; y_{t-1}\right) \\
-D_{3} g\left(y_{t-1}, \xi_{t-1} ; y_{t}\right)
\end{array}\right] .
\end{aligned}
$$

These FOCs are necessarily satisfied by the optimal equilibrium, if one exists, but a solution to the FOCs is not necessarily an optimum. We shall assume, however, that an optimum satisfying the FOCs does exist; and under further assumptions stated below, a bounded solution to the FOCs will also necessarily correspond to an optimum.

Note that (2.6) is also the Lagrangian for an alternative problem, the problem of choosing a path $\left\{y_{t}\right\}$ to maximize the modified objective

$$
V_{t_{0}}+\beta^{-1} \Theta_{t_{0}-1}^{\prime} g\left(y_{t_{0}-1}, \xi_{t_{0}-1} ; y_{t_{0}}\right)
$$

subject to the constraints $(2.2)-(2.4)$ for dates $t \geq t_{0}$. In this alternative formulation, the vector $\Theta_{t_{0}-1}$ is part of the data defining the problem, just as the vector $\bar{g}_{t_{0}}$ is in the problem stated earlier. Since the Lagrangian for this alternative problem is the same, the set of first-order conditions is also identical to the ones given above. Therefore, any solution to the first type of problem for some choice of the vector $\bar{g}_{t_{0}}$ is also a solution to a problem of the second type for some choice of the vector $\Theta_{t_{0}-1}$, and vice versa. The modification of the policy authority's objective as in (2.9) is an alternative way of requiring it to take account of the value to a policymaker at an earlier time of being able to anticipate a particular kind of policy from date $t_{0}$ onward. ${ }^{14}$

The fact that $\varphi_{2, t}=\Theta_{t-1}$ means that these multipliers are determined a period in advance (unlike the elements of $\varphi_{1, t}$ ). Hence a further requirement for consistency of the multipliers $\left\{\varphi_{t}\right\}$

\footnotetext{
${ }^{14}$ This alternative way of requiring the policymaker to internalize the consequences of a prior commitment is used, for example, in Marcet and Marimon (1998), Khan et al. (2003), and Svensson and Woodford (2005).
} 
with an optimal plan is that

$$
\varphi_{2, t}-E_{t-1} \varphi_{2, t}=0
$$

for all $t>t_{0}$. In addition, $\varphi_{2, t_{0}}$ is given as an initial condition (in the case of the modified problem defined by a vector of initial multipliers $\left.\Theta_{t_{0}-1}\right)$. Alternatively, we adjoin an initial pre-commitment (2.5) to the set of structural equations, and leave the vector $\varphi_{2, t_{0}}$ to be determined endogenously.

\subsection{Optimal Steady State}

If an optimal steady state (an optimum in which $y_{t}=\bar{y}$ for all $t$, under the assumption that $\xi_{t}=\bar{\xi}$ for all $t$ ) exists, it will be described by constant vectors $(\bar{y}, \bar{\varphi}, \bar{\xi})$ satisfying

$$
\begin{aligned}
F(\bar{y}, \bar{\xi} ; \bar{y}) & =0, \\
g(\bar{y}, \bar{\xi} ; \bar{y}) & =0, \\
\left(D_{1} \pi\right)^{\prime}+\left(\bar{A}-\beta^{-1} \bar{I}\right)^{\prime} \bar{\varphi} & =0,
\end{aligned}
$$

where $D_{1} \pi \equiv D_{1} \pi(\bar{y}, \bar{\xi}), D_{j} F \equiv D_{j} F(\bar{y}, \bar{\xi} ; \bar{y}), D_{j} g \equiv D_{j} g(\bar{y}, \bar{\xi} ; \bar{y})$ for $j=1,2,3$, and

$$
\begin{aligned}
\bar{A} & \equiv \bar{A}(\bar{y}, \bar{y}, \bar{\xi}, \bar{\xi}), \\
\bar{I} & \equiv \bar{I}(\bar{y}, \bar{y}, \bar{\xi}, \bar{\xi}) .
\end{aligned}
$$

We shall suppose that such a steady state exists; in fact, we make the more specific assumption stated below.

Assumption 1 (a) Let a vector $\bar{\xi}$ of "steady state" values of the exogenous disturbances be given. Then there exist vectors $\bar{y} \in \mathbf{R}^{m}$ and $\bar{\varphi} \in \mathbf{R}^{n}$ such that if $y_{t_{0}-1}=\bar{y}, \Theta_{t_{0}-1}=\bar{\Theta}\left[\equiv \bar{\varphi}_{2}\right], \bar{F}_{t_{0}}=0$ and $\xi_{t}=\bar{\xi}$ with certainty for all $t \geq t_{0}-1$, the optimal policy [the one that maximizes (2.9) subject to constraints (2.2)-(2.4)] involves $y_{t}=\bar{y}$ with certainty for all $t \geq t_{0}$.

(b) Moreover, for any small enough neighborhood $\mathcal{N}_{y}$ of $\bar{y}$, there exist neighborhoods $\mathcal{N}_{\Theta}$ of $\bar{\Theta}, \mathcal{N}_{\theta}$ of $\bar{\theta}, \mathcal{N}_{F}$ of 0 , and $\mathcal{N}_{2} \subseteq \mathcal{N}_{y}$ such that for any $y_{t_{0}-1} \in \mathcal{N}_{2}, \Theta_{t_{0}-1} \in \mathcal{N}_{\Theta}, \bar{F}_{t_{0}} \in \mathcal{N}_{F}$, and a disturbance vector $\xi_{t}=\bar{\xi}$ for all $t \geq t_{0}-1$, the optimal policy involves $y_{t} \in \mathcal{N}_{y}$ with $y_{t} \rightarrow \bar{y}$ as $t \rightarrow \infty$. Moreover, there exists a sequence of multipliers $\left\{\varphi_{t}\right\}$ consistent both with the specified initial conditions and with the FOCs (2.8) for all $t \geq t_{0}$, such that $\varphi_{t} \in \mathcal{N}_{\Theta} \times \mathcal{N}_{\theta}$ for all $t \geq t_{0}$, and $\varphi_{t} \rightarrow \bar{\varphi}$ as $t \rightarrow \infty$. Finally, the convergence is exponential: there exists $\delta>1$ such that

$$
\lim _{t \rightarrow \infty} \delta^{t}\left(y_{t}-\bar{y}\right)=0, \quad \lim _{t \rightarrow \infty} \delta^{t}\left(\varphi_{t}-\bar{\varphi}\right)=0
$$


for any initial conditions in the specified set.

(c) There also exists a neighborhood $\mathcal{N}_{g}$ of the vector $\bar{g} \equiv g(\bar{y}, \bar{\xi} ; \bar{y})$ and a diffeomorphism

$$
\gamma: \mathcal{N}_{\Theta} \times \mathcal{N}_{2} \times \mathcal{N}_{F} \rightarrow \mathcal{N}_{g} \times \mathcal{N}_{2} \times \mathcal{N}_{F}
$$

with the properties

$$
\gamma_{2}(\Theta, y, \bar{F})=y, \quad \gamma_{3}(\Theta, y, \bar{F})=\bar{F},
$$

such that for any $\Theta_{t_{0}-1} \in \mathcal{N}_{\Theta}$, a path $\left\{y_{t}\right\}$ for $t \geq t_{0}$ maximizes (2.9) in the case of this vector of multipliers, initial conditions $\left(y_{t_{0}-1}, \bar{F}_{t_{0}}\right)$, and a specification that $\xi_{t}=\bar{\xi}$ for all $t$, if and only if it maximizes (2.1) subject to the initial pre-commitments (2.5), where $\bar{g}_{t_{0}}=\gamma_{1}\left(\Theta_{t_{0}-1}, y_{t_{0}-1}, \bar{F}_{t_{0}}\right)$, and the same initial conditions $\left(y_{t_{0}-1}, \bar{F}_{t_{0}}\right)$. Moreover, the matrix $\bar{\Gamma} \equiv D_{1} \gamma_{1}(\bar{\Theta}, \bar{y}, 0)$ is non-singular.

Assumption 1 implies not only that a steady state exists, but that in the case of any initial conditions close enough to consistency with the steady state and no stochastic disturbances, the optimal equilibrium will remain forever near this steady state, and converge to it asymptotically (indeed, at an exponential rate). We also assume that the Lagrange multipliers associated with the optimal policy remain forever near their steady-state values and converge exponentially as well. It then follows that for any small enough neighborhoods of the steady state (as specified in the statement of the assumption), there must exist a neighborhood $\mathcal{N}_{\xi}$ of $\bar{\xi}$, such that the optimal dynamics will also involve $y_{t} \in \mathcal{N}_{y}$ and $\varphi_{t} \in \mathcal{N}_{\Theta} \times \mathcal{N}_{\theta}$ for all $t \geq t_{0}$ as long as $\xi_{t} \in \mathcal{N}_{\xi}$ for all $t \geq t_{0}-1$, though of course there is no convergence to the steady state in the stochastic case. This makes it possible for us to analyze optimal stabilization policy in response to small enough disturbances through a purely local analysis of equilibria in which the endogenous variables $y_{t}$ remain forever near the steady-state values $\bar{y}$ and the multipliers $\left\{\varphi_{t}\right\}$ remain forever near the steady-state values $\bar{\varphi}$.

Assumption 1(c) implies that in the case of all initial conditions close enough to consistency with the steady state (which is to say, all of those with which we shall be concerned in this paper), there is a unique problem of the first type (defined by a vector of pre-commitments $\bar{g}_{t_{0}}$ ) corresponding to each problem of the second type (defined by a vector of initial Lagrange multipliers $\Theta_{t_{0}-1}$ ), and vice versa. The additional stipulation that the mapping $\gamma$ has a non-singular Jacobian matrix is only a slight strengthening of the assumption that the mapping is invertible near the steady state; this is necessary to allow us to assert a similar equivalence between the linearized versions of the conditions that characterize the two kinds of optimal plans. 
Assumption 1 implies the existence of steady-state values $(\bar{y}, \bar{\varphi})$ that satisfy conditions $(2.13)$, associated with the given stationary vector of values $\bar{\xi}$ for the exogenous states, and further implies the existence of a solution to the FOCs, in which the variables $y_{t}$ and $\varphi_{t}$ remain forever near their steady-state values, in the case of any small enough perturbations in the initial conditions. It is these local solutions to the FOCs (2.8) that we wish to further characterize, through the linear approximation presented in the next section. Under relatively weak regularity conditions, we show that there must be at most one solution to the FOCs that remains forever near the steady state, which solution must (by Assumption 1) be the optimal equilibrium. Hence it suffices to find a target criterion with the property that a path for the endogenous variables that is feasible under some policy, that remains forever within the neighborhood $\mathcal{N}_{y}$, and that fulfills the target criterion at all times (i) exists in the case of all initial conditions near enough to consistency with the steady state and all small enough disturbances, and (ii) must also satisfy the FOCs in all periods, in order for us to conclude that fulfillment of the target criterion is necessary and sufficient for the intended future evolution of policy to be optimal, again in the case of all initial conditions near enough to consistency with the steady state and all small enough disturbances.

We do not here seek to provide more fundamental assumptions under which Assumption 1 is necessarily valid. In practice it is relatively straightforward in applications to verify the existence of a steady-state solution to the FOCs (2.13). This will then provide a steady-state solution of the kind assumed, in the case of an initial precommitment with the "self-consistency" property. It is also straightforward to evaluate the second-order conditions [SOCs] that tell whether this solution represents a local welfare optimum, discussed further in section 3.2.1 below, and to analyze convergence to the steady state in the deterministic case for initial conditions close enough to consistency with the steady state, as these involve algebraic properties of the derivatives of the functions defining the problem, evaluated at the steady-state values of their arguments. The more difficult question is verification of the global optimality of the local optimum represented by a solution to the FOCs and SOCs. We do not address this here, but assume that it is satisfied in an application of interest.

Finally, note that while we assume the existence of an "optimal steady state" in the sense that optimal policy involves constant values of the endogenous variables in the absence of random disturbances, we do not assume that the steady state near which we conduct our analysis is an undistorted steady state. That is, we do not assume that in the absence of disturbances and under suitable initial conditions, there would exist a policy that achieves the unconstrained maximum 
of the function $\pi(y ; \bar{\xi})$ each period. Our methods are applicable to problems in which there exist distortions, the severity of which depends on policy, but that cannot be eliminated at all times by any policy. For example, in the application treated in section 4, the steady-state level of output may be inefficiently low, owing either to market power or to tax distortions, even under the optimal monetary policy.

\section{Linear Approximation of the Optimal Equilibrium Dynamics}

As long as we are interested solely in small enough perturbations of the steady-state policy problem, of the kind described in Assumption 1, we can approximate the optimal solution for $y_{t}$ as a function of the initial conditions $\left(y_{t_{0}-1}, \xi_{t_{0}-1}, \bar{F}_{t_{0}}, \Theta_{t_{0}-1}\right)$ and the history of disturbances $\left(\xi_{t_{0}}, \ldots, \xi_{t}\right)$ by a linear function of these variables, where the coefficient on each variable represents the partial derivative of the optimal $y_{t}$ with respect to that variable, with all partial derivatives evaluated at the steady state referred to in Assumption 1. And as usual, the implicit function theorem allows us to calculate these derivatives by solving linearized versions of the conditions that describe an optimum, where the linearizations are all computed at the steady-state solution. ${ }^{15}$

\subsection{Approximation}

Local approximation of the optimal equilibrium dynamics requires us to solve a system of linear difference equations consisting of linearizations of two sets of nonlinear equations: the structural equations (2.2)-(2.4) on the one hand, and the first-order conditions (2.8) on the other. We linearize these equations around the steady-state values of the arguments $\left(y_{t}=\bar{y}, \varphi_{t}=\bar{\varphi}\right.$ and $\xi_{t}=\bar{\xi}$ for all $t$ ), where $\bar{y}$ and $\bar{\varphi}$ are the optimal steady state values (assumed to exist in Assumption 1) in the case of the constant vector $\bar{\xi}$ for the exogenous variables. We will denote the deviations from steady-state values by $\tilde{y}_{t} \equiv y_{t}-\bar{y}, \tilde{\varphi}_{t} \equiv \varphi_{t}-\bar{\varphi}, \tilde{\xi}_{t} \equiv \xi_{t}-\bar{\xi}$, and derive local approximations for the case in which these terms are small. Specifically, we consider the family of stochastic processes

$$
\xi_{t}=\bar{\xi}+\epsilon u_{t}
$$

indexed by a parameter $\epsilon \geq 0$, where $\left\{u_{t}\right\}$ is some bounded process (held fixed as we consider variations in $\epsilon$ ). We wish to characterize optimal monetary policy for the case in which $\epsilon$ is sufficiently small (though still positive), by computing solutions for the state-contingent evolution

\footnotetext{
${ }^{15}$ See Woodford (2003, Appendix A.3) or Benigno and Woodford (2012) for further discussion.
} 
of all endogenous variables that are accurate up to an error that is at most of size $\mathcal{O}\left(\epsilon^{2}\right)$. To economize on notation, we shall assume that the disturbances $\left\{\xi_{t}\right\}$ evolve in accordance with a linear first-order vector autoregressive process, ${ }^{16}$ so that

$$
E_{t} \tilde{\xi}_{t+1}=\varrho \tilde{\xi}_{t}
$$

where $\varrho$ is a matrix such that $\|\varrho\|<1$.

The following Lemma summarizes the linear approximations of the structural equations and first-order conditions.

Lemma 1 Assuming that the exogenous disturbances satisfy (3.1), a first-order approximation of the structural equations (2.2)-(2.3) around the steady-state can be written as

$$
\bar{I} E_{t} \tilde{y}_{t+1}=\bar{A} \tilde{y}_{t}+\bar{C} \tilde{\xi}_{t}
$$

for all $t \geq t_{0}$, omitting terms that are merely of second order,

$$
\bar{I}_{1}\left(\tilde{y}_{t}-E_{t-1} \tilde{y}_{t}\right)=\bar{D}\left(\tilde{\xi}_{t}-E_{t-1} \tilde{\xi}_{t}\right)
$$

for all $t>t_{0}$, where $\bar{I}$ and $\bar{A}$ are the same matrices as in (2.13), $\bar{I}_{1} \equiv-\beta D_{1} F, \bar{A}_{1} \equiv \beta D_{3} F$ are the $k \times m$ matrices consisting of the first $k$ rows of $\bar{I}$, respectively $\bar{A}$, and

$$
\bar{C} \equiv\left[\begin{array}{c}
\beta D_{2} F \varrho \\
D_{2} g
\end{array}\right], \quad \bar{D} \equiv \beta D_{2} F .
$$

The linearized version of the initial conditions (2.4) can be expressed as

$$
\bar{I}_{1} \tilde{y}_{t_{0}}=\bar{A}_{1} \tilde{y}_{t_{0}-1}+\bar{D} \tilde{\xi}_{t_{0}}-\beta \bar{F}_{t_{0}}
$$

Similarly, a linear approximation of the nonlinear first-order conditions (2.8) takes the form

$$
0=E_{t}\left[\left(\beta R^{\prime}+S L+R L^{2}\right) \tilde{y}_{t+1}\right]+E_{t}\left[\left(\bar{A}-\beta^{-1} \bar{I} L\right)^{\prime} \tilde{\varphi}_{t+1}\right]+E_{t}\left[B(L) \tilde{\xi}_{t+1}\right]
$$

where $L$ denotes the lag operator and we define

$$
\begin{aligned}
R \equiv & \left(\bar{\theta}^{\prime} \otimes I_{m}\right) D_{3}\left[\left(D_{1} F\right)^{\prime}\right]+\left(\bar{\Theta}^{\prime} \otimes I_{m}\right) \beta^{-1} D_{1}\left[\left(D_{3} g\right)^{\prime}\right], \\
S \equiv & D_{1}\left[\left(D_{1} \pi\right)^{\prime}\right]+\left(\bar{\theta}^{\prime} \otimes I_{m}\right)\left\{D_{1}\left[\left(D_{1} F\right)^{\prime}\right]+\beta D_{3}\left[\left(D_{3} F\right)^{\prime}\right]\right\} \\
& +\left(\bar{\Theta}^{\prime} \otimes I_{m}\right)\left\{D_{1}\left[\left(D_{1} g\right)^{\prime}\right]+\beta^{-1} D_{3}\left[\left(D_{3} g\right)^{\prime}\right]\right\},
\end{aligned}
$$

\footnotetext{
${ }^{16}$ Note that this allows for serial correlation of arbitrary complexity in the disturbance processes, as long as the vector $\xi_{t}$ is assumed to include a sufficient number of lagged disturbances.
} 
and

$$
\begin{aligned}
B(L) \equiv & \left\{D_{2}\left[\left(D_{1} \pi\right)^{\prime}\right]+\left(\bar{\theta}^{\prime} \otimes I_{m}\right) D_{2}\left[\left(D_{1} F\right)^{\prime}\right]+\left(\bar{\Theta}^{\prime} \otimes I_{m}\right) D_{2}\left[\left(D_{1} g\right)^{\prime}\right]\right\} \cdot L \\
& +\beta^{-1}\left(\bar{\Theta}^{\prime} \otimes I_{m}\right) D_{2}\left[\left(D_{3} g\right)^{\prime}\right] \cdot L^{2}+\beta\left(\bar{\theta}^{\prime} \otimes I_{m}\right) D_{2}\left[\left(D_{3} F\right)^{\prime}\right]
\end{aligned}
$$

Finally, a linear approximation of (2.10) yields

$$
\tilde{\varphi}_{2, t}-E_{t-1} \tilde{\varphi}_{2, t}=0
$$

for all $t>t_{0}$.

Note that the matrices $\bar{I}, \bar{A}, \bar{I}_{1}, \bar{A}_{1}, \bar{D}, S, R$, and the matrix polynomial $B(L)$ are independent of the specification of the shock processes, and in particular of the matrix $\varrho$. The proof of the lemma is in Appendix B.1.

\subsection{Regularity Conditions}

Our analysis relies upon some further properties of the matrices of coefficients of the linearized structural relations. Here we state these additional assumptions and discuss some of their implications. The assumptions are not too restrictive, but allow us to simplify our analysis and the statement of our results in important respects.

The linearized structural equations are valid only locally, so that it makes sense to consider only solutions to these equations that are bounded, i.e., that satisfy the bound

$$
\|\tilde{y}\| \equiv \operatorname{ess} \sup _{t \geq t_{0}}\left|\tilde{y}_{t}\right|<\infty .
$$

We shall state our further regularity conditions in terms of the matrices $\bar{I}$ and $\bar{A}$.

Assumption 2 (a) The matrix

$$
\left[\begin{array}{l}
\bar{I} \\
\bar{A}
\end{array}\right]
$$

is of full column rank, i.e., of rank $m$.

(b) In addition, the matrix pencil $\bar{I}-\mu \bar{A}$ is of rank $n$. Hence its rows are linearly independent; that is, there exists no vector $\varphi(\mu) \neq 0$ of polynomial functions of $\mu$ such that

$$
\varphi(\mu)^{\prime}[\bar{I}-\mu \bar{A}]=0
$$

for all $\mu$. Moreover, there exists no vector $\bar{\varphi} \neq 0$ such that

$$
\bar{\varphi}^{\prime}[\bar{I}-\beta \bar{A}]=0 .
$$


Assumption 2(a) requires that $m \leq 2 n$, as mentioned earlier; thus the number of independent dimensions along which policy can be varied is not too great, relative to the number of dimensions of variation that are possible in the endogenous variables. However, in the case that $m \leq 2 n$, the assumption holds for generic matrices of coefficients $\bar{I}$ and $\bar{A}$. If Assumption 2(a) did not hold, the vector $y_{t}$ would involve redundant state variables, that could be eliminated altogether, as far as the structural equations (3.2) are concerned. ${ }^{17}$ Assumption 2(a) could equivalently be stated as a property of the pencil $\bar{I}-\mu \bar{A}$ : there exists no constant vector $y \neq 0$ such that

$$
[\bar{I}-\mu \bar{A}] y=0
$$

for all $\mu .^{18}$

Assumption 2(b) similarly implies that there is no redundancy among the separate structural equations (3.2); it is a stronger form of linear independence assumption, since it also excludes the possibility that some equation in the system (3.2) is implied by conditional expectations of leads of other equations in the system. The further stipulation in the last sentence is the algebraic condition required for uniqueness of the steady-state Lagrange multipliers $\bar{\varphi}$ associated with the optimal steady state $\bar{y}$ around which we conduct our local analysis.

\subsubsection{Second-Order Conditions}

We shall make use of a further regularity condition, involving the matrices $R$ and $S$, in addition to the regularity conditions involving the matrices $\bar{I}$ and $\bar{A}$ stated above. This is a second-order condition for the optimality of the steady state that represents a slight strengthening of the conditions already implicit in Assumption 1.

Let $\left\{y_{i}\right\}$ be a bounded sequence of vectors of dimension $m$, not all equal to zero, such that

$$
\bar{I} y_{0}=0, \quad \bar{A} y_{i}-\bar{I} y_{i+1}=0 \quad \text { for all } i \geq 0 .
$$

Then let us consider a perturbation of the path $\left\{\tilde{y}_{t}\right\}$, replacing $\tilde{y}_{\tau+i}$ with

$$
\tilde{y}_{\tau+i}+\varepsilon y_{i}
$$

\footnotetext{
${ }^{17}$ These variables may not actually be redundant, because they might nonetheless matter for equations (3.3)-(3.4); but we abstract from this complication here, and instead assume that it is possible to write the system in terms of a vector of $m$ endogenous variables for which Assumption 2(a) is satisfied.

${ }^{18} \mathrm{But}$ it is not possible to make a similar statement about the nonexistence of polynomial solutions; for the fact that $m>n$ implies that there must exist polynomial functions $y(\mu)$ such that $[\bar{I}-\mu \bar{A}] y(\mu)=0$ for all $\mu$. Assumption 2(a) implies only that all such solutions must be of order greater than zero in $\mu$.
} 
for all $i \geq 0$ and an arbitrary date $\tau \geq t_{0}$, while leaving $\tilde{y}_{t}$ unchanged for all dates $t<\tau$. Here $\varepsilon$ is an arbitrary small quantity. The path $\left\{\tilde{y}_{t}\right\}$ remains consistent with the linearized structural equations (3.2)-(3.4), and continues to satisfy the bound $\|\tilde{y}\|<\infty$, for any value of $\varepsilon$. Then for any small enough value of $|\varepsilon|$, this also approximates a feasible perturbation under the exact structural relations (2.2)-(2.4), up to an error of order $\mathcal{O}\left(|\varepsilon|^{2}\right)$.

In order for the original path $\left\{\tilde{y}_{t}\right\}$ to have been optimal, no such perturbation can increase the value of the Lagrangian (2.6). In particular, given our assumption of the optimality of the steady state in which $y_{t}=\bar{y}$ for all $t$, no such perturbation of the steady state can increase the value of the Lagrangian. In the case of any small enough value of $|\varepsilon|$, the increase in the value of the Lagrangian as a result of the perturbation $\left\{y_{i}\right\}$ is proportional to

$$
\sum_{i=0}^{\infty} \beta^{i}\left[y_{i}^{\prime} S y_{i}+y_{i+1}^{\prime} \beta R y_{i}+y_{i}^{\prime} \beta R^{\prime} y_{i+1}\right]|\varepsilon|^{2}+\mathcal{O}\left(|\varepsilon|^{3}\right)
$$

regardless of the date $\tau,{ }^{19}$ where all derivatives are evaluated at the steady state. Hence a sufficient condition for such a perturbation of the steady-state policy to be welfare-reducing, in the case of any small enough $|\varepsilon|>0$, is that

$$
\sum_{i=0}^{\infty} \beta^{i}\left[y_{i}^{\prime} S y_{i}+y_{i+1}^{\prime} \beta R y_{i}+y_{i}^{\prime} \beta R^{\prime} y_{i+1}\right]<0 .
$$

We can now state the additional regularity condition that we shall invoke.

Assumption 3 In the case of any non-zero bounded sequence $\left\{y_{i}\right\}$ such that (3.11) is satisfied, (3.12) also holds.

These are second-order conditions for the optimality of the steady state defined by $(2.13) .{ }^{20}$ Technically, Assumption 3 is not implied by Assumption 1 as stated above, as that would only require a weak inequality in (3.12). The present assumption is, however, only a slight strengthening of the one already implicit in Assumption 1.

\subsection{Determinacy of the Solution to the FOCs}

We now show that there is a unique bounded (or determinate) solution to the nonlinear FOCs for the optimal policy problem. We have already assumed that there exists a bounded optimal

\footnotetext{
${ }^{19}$ See Appendix B.2 for details of this calculation.

${ }^{20}$ Benigno and Woodford (2012) present an alternative formulation of the SOCs that has some advantages from a computational standpoint.
} 
equilibrium path (Assumption 1), and since the FOCs are necessary for optimality, there must exist a bounded solution to the FOCs. The goal of the present section is to show that there can be only one bounded solution to the FOCs. It will follow from this result that in the case of a bounded solution, the FOCs are also sufficient for optimality.

As usual, for the analysis of determinacy it suffices that we demonstrate the existence of a unique bounded solution to the linearized versions of the equations for which we seek to demonstrate the existence of a locally unique solution. We therefore consider the system of equations consisting of the linearized structural equations (3.2)-(3.4) together with the linearized FOCs (3.5)-(3.6).

We now state our first important result.

Proposition 1 Suppose that Assumptions 1, 2(b) and 3 are satisfied. Then for any initial conditions $\tilde{y}_{t_{0}-1}, \bar{F}_{t_{0}}$, any specification of the initial Lagrange multipliers $\tilde{\Theta}_{t_{0}-1}$, and any exogenous disturbance process $\left\{\tilde{\xi}_{t}\right\}$ satisfying $\|\tilde{\xi}\|<\infty$ where the norm is defined as in (3.7), there exists a unique solution to the system consisting of the linearized structural equations (3.2)-(3.4) and the FOCs (3.5)-(3.6) for all $t \geq t_{0}$ with the property that $\|\tilde{y}\|<\infty$ and $\|\tilde{\varphi}\|<\infty$.

The proof is in Appendix A.1. Note that this unique solution to the linearized FOCs represents a local linear approximation to an optimal plan, that is, to a plan that coincides (up to an error of order $\left.\mathcal{O}\left(\epsilon^{2}\right)\right)$ with a plan that maximizes the modified objective function (2.9) defined by the initial multipliers $\tilde{\Theta}_{t_{0}-1}$, for the specified initial conditions. Hence any solution to the system of FOCs with the property that $\|\tilde{y}\|<\infty$ represents a local linear approximation to an optimal plan.

Using Assumption 1(c), we immediately obtain as a further corollary that there also exists a unique bounded solution to the system of FOCs augmented by the linearization of the initial precommitments (2.5), for any choice of $\bar{g}_{t_{0}}$, and that any bounded solution to this system of FOCs represents a local linear approximation to a plan that is optimal in the sense of maximizing (2.1) subject to the initial pre-commitments. These results follow immediately from Proposition 1, given the existence of an invertible linear relationship between specifications of $\Theta_{t_{0}-1}$ and specifications of $\bar{g}_{t_{0}}$.

\section{The Optimal Target Criterion}

We now turn to the main topic of our paper, the construction of an optimal target criterion. Our task is to show that there exists a criterion that, when conjoined with the linearized structural 
relations (3.2)-(3.4), determines a unique bounded solution for the endogenous variables $\left\{\tilde{y}_{t}\right\}$, and that the equilibrium evolution that it determines represents a local linear approximation to an optimal plan, by which we mean a plan that maximizes (2.9) for some initial Lagrange multipliers $\Theta_{t_{0}-1}$ that are determined by a self-consistent rule, or that maximizes (2.1) subject to some initial pre-commitments (2.5) determined by a self-consistent rule. Our method is to construct a target criterion, involving only the endogenous variables $\left\{\tilde{y}_{t}\right\}$, that is fulfilled each period if and only if the linearized FOCs are satisfied, where the FOCs in question are those associated with some self-consistent selection of the initial Lagrange multipliers or of the initial pre-commitments. Then the determinacy of equilibrium under the target criterion will follow from Proposition 1, as will the fact that the equilibrium so determined represents a local linear approximation to an equilibrium that is optimal from a timeless perspective.

\subsection{Target Variables}

Let us recall that the linearized FOCs (3.5)-(3.6) can be written in the form

$$
\begin{aligned}
E_{t}\left[\left(\bar{A}-\bar{I} \beta^{-1} L\right)^{\prime} \tilde{\varphi}_{t+1}\right] & =\tau_{t}-\tau_{t}^{*} \\
\tilde{\varphi}_{2, t}-E_{t-1} \tilde{\varphi}_{2, t} & =0
\end{aligned}
$$

where we now define a vector of (endogenous) "target variables"

$$
\tau_{t} \equiv-E_{t}\left[\left(\beta R^{\prime}+S L+R L^{2}\right) \tilde{y}_{t+1}\right]
$$

and time-varying (exogenous) "target values" for them

$$
\tau_{t}^{*} \equiv E_{t}\left[B(L) \tilde{\xi}_{t+1}\right]
$$

Recall also that (4.1) must hold for all $t \geq t_{0}$, while (4.2) must hold for all $t>t_{0}$. We add in addition the requirement that $\tilde{\varphi}_{2, t_{0}}$ be equal to some given vector of initial multipliers $\tilde{\Theta}_{t_{0}-1}$, unless we add linearized initial pre-commitments of the form (2.5) to determine $\tilde{\varphi}_{2, t_{0}}$.

Here the target variables $\tau_{t}$ summarize the ways in which variations in the endogenous variables $\tilde{y}_{t}$ are relevant to verification of whether the FOCs are satisfied or not; hence policy need only be concerned with the projected paths of these particular variables. Moreover, the target variables only enter the FOCs through their difference with respect to the exogenously varying target values $\tau_{t}^{*}$; hence policy need only be concerned with the extent to which the target variables are projected to differ from their respective target values. 
In general, the target variables may be linear combinations of the entire vector of endogenous variables $\tilde{y}_{t}$. However, there is one case of practical interest in which the target variables depend only on the variables entering the policymaker's objective function.

\subsubsection{Special Case: Quadratic Objective Function}

Consider the case in which the policy objective is a quadratic function

$$
\pi\left(y_{t} ; \xi_{t}\right)=-\frac{1}{2}\left(q_{t}-q_{t}^{*}\right)^{\prime} W\left(q_{t}-q_{t}^{*}\right)
$$

where $W$ is a symmetric, positive definite matrix, where $q_{t} \equiv w^{\prime} y_{t}$ is a subset of the endogenous variables and $q_{t}^{*} \equiv q^{*}\left(\xi_{t}\right)$ is a vector of exogenous targets for the variables $q_{t}$. The matrix $w^{\prime}$ is $m_{\tau} \times m$, where $m_{\tau}$ may be much smaller than $m$, in the case of a policy institution with a fairly complex model of the economy but a relatively simple stabilization objective (that may, for example, reflect its legislative mandate).

Let us suppose furthermore that the targets are achievable in steady state; this means that in the case that $\xi_{t}=\bar{\xi}$ at all times, there would exist a constant vector $\bar{y}$ of values for the endogenous variables satisfying $(2.11)-(2.12)$ and

$$
w^{\prime} \bar{y}=q^{*}(\bar{\xi})
$$

In this case, a simple characterization of the target variables and target gaps is possible.

Lemma 2 Suppose that the objective of policy is a quadratic function (4.3), that the targets $q^{*}\left(\xi_{t}\right)$ are achievable in steady state, in the sense that there exists a vector $\bar{y}$ satisfying conditions (2.11)(2.12) and (4.4), and that Assumption 2(b) is satisfied. Then the target variables $\tau_{t}$ are all linear combinations of the $m_{\tau}$ variables $q_{t}-\bar{q}$, where $\bar{q} \equiv w^{\prime} \bar{y}$ are the steady-state values of the variables $q_{t}$. Moreover, the "target gaps" appearing on the right-hand side of (4.1) are equal to

$$
\tau_{t}-\tau_{t}^{*}=w W\left(q_{t}-q_{t}^{*}\right)+\mathcal{O}\left(\epsilon^{2}\right)
$$

to first order, which means that the linearized FOCs (4.1) can be expressed entirely in terms of the $m_{\tau}$ target variables $\left\{q_{t}-q_{t}^{*}\right\}$ and the Lagrange multipliers $\left\{\tilde{\varphi}_{t}\right\}$.

The proof is in Appendix A.2. In this special case, at least, the optimal target criterion can be expressed purely as a restriction upon the evolution of the $m_{\tau}$ target variables $\left\{q_{t}-q_{t}^{*}\right\}$ that enter the objective function. 


\subsubsection{General Case}

Regardless of whether the special assumptions required for Lemma 2 are satisfied, it will be useful to express the linearized FOCs (4.1) in a canonical form, by applying a Kronecker canonical decomposition to the matrix pencil $(\bar{A}-\mu \bar{I})^{\prime}$.

Lemma 3 Under Assumption 2 there necessarily exist non-singular matrices $P(n \times n)$ and $Q$ $(m \times m)$ such that

$$
(\bar{A}-\mu \bar{I})^{\prime}=Q\left[\begin{array}{cc}
B_{1}^{\prime}-\mu J_{1}^{\prime} & 0 \\
0 & B_{2}^{\prime}-\mu J_{2}^{\prime}
\end{array}\right] P
$$

where $B_{1}^{\prime}-\mu J_{1}^{\prime}$ is a matrix pencil of dimension $(n-q) \times(m-q)$ and $B_{2}^{\prime}-\mu J_{2}^{\prime}$ is a regular pencil of dimension $q \times q$, for some $q$ satisfying $0 \leq q<n$. The pencil $B_{1}^{\prime}-\mu J_{1}^{\prime}$ takes the form

$$
B_{1}^{\prime}-\mu J_{1}^{\prime}=\left[\begin{array}{ccc}
M_{1}(\mu) & \cdots & 0 \\
\vdots & \ddots & \vdots \\
0 & \cdots & M_{p}(\mu)
\end{array}\right]
$$

where $p=m-n \geq 1$, and where for each $i=1, \ldots, p, M_{i}(\mu)$ is an $\left(\epsilon_{i}+1\right) \times \epsilon_{i}$ matrix pencil of the form

$$
M_{i}(\mu) \equiv\left[\begin{array}{cccc}
\mu & 0 & \cdots & 0 \\
1 & \mu & & \vdots \\
0 & 1 & \ddots & 0 \\
\vdots & & \ddots & \mu \\
0 & 0 & \cdots & 1
\end{array}\right]
$$

for some $\epsilon_{i} \geq 1$. Moreover,

$$
q=n-\sum_{i=1}^{p} \epsilon_{i}
$$

The proof is in Appendix A.3.

This result allows the system of FOCs (4.1) to be equivalently expressed in the form

$$
\begin{aligned}
E_{t}\left[M_{i}\left(\beta^{-1} L\right) \hat{\varphi}_{1 i, t+1}\right] & =\hat{\tau}_{1 i, t}, \quad(i=1, \ldots, p) \\
B_{2}^{\prime} E_{t} \hat{\varphi}_{2, t+1}-\beta^{-1} J_{2}^{\prime} \hat{\varphi}_{2, t} & =\hat{\tau}_{2, t},
\end{aligned}
$$


where we partition the vectors

$$
P \tilde{\varphi}_{t} \equiv \hat{\varphi}_{t}=\left[\begin{array}{c}
\hat{\varphi}_{11, t} \\
\vdots \\
\hat{\varphi}_{1 p, t} \\
\hat{\varphi}_{2, t}
\end{array}\right], \quad Q^{-1}\left(\tau_{t}-\tau_{t}^{*}\right) \equiv \hat{\tau}_{t}=\left[\begin{array}{c}
\hat{\tau}_{11, t} \\
\vdots \\
\hat{\tau}_{1 p, t} \\
\hat{\tau}_{2, t}
\end{array}\right]
$$

conformably with the partition of the matrix in (4.6). We thus reduce the FOCs to separate blocks of equations relating subsets of the Lagrange multipliers $\hat{\varphi}_{1, t}^{\prime}=\left[\hat{\varphi}_{11, t}, \ldots, \hat{\varphi}_{1 p, t}\right]$, and $\hat{\varphi}_{2, t}$ to particular subsets of the target variables $\hat{\tau}_{1, t}^{\prime}=\left[\hat{\tau}_{11, t}, \ldots, \hat{\tau}_{1 p, t}\right]$, and $\hat{\tau}_{2, t}$. Furthermore, each of the blocks of equations in (4.8) has a particular simple form.

For generic $n \times m$ matrices $\bar{A}$ and $\bar{I}$, the decomposition (4.6) furthermore involves $q=0$. In this case, the $q \times 1$ vectors $\hat{\varphi}_{2, t}$ and $\hat{\tau}_{2, t}$ vanish, and the system of FOCs (4.1) is equivalent to (4.8). Furthermore, the system of FOCs (4.2) can be written equivalently as

$$
0=\tilde{\varphi}_{2, t}-E_{t-1} \tilde{\varphi}_{2, t}=\left[\begin{array}{ll}
0 & I_{n-k}
\end{array}\right]\left(\tilde{\varphi}_{t}-E_{t-1} \tilde{\varphi}_{t}\right)=\left[\begin{array}{ll}
0 & I_{n-k}
\end{array}\right] P^{-1}\left(\hat{\varphi}_{1, t}-E_{t-1} \hat{\varphi}_{1, t}\right)
$$

or

$$
0=V_{2}\left(\hat{\varphi}_{1, t}-E_{t-1} \hat{\varphi}_{1, t}\right)
$$

where the matrix $V_{2}$ is given by a linear combination of the last $n-k$ rows of $P^{-1}: V_{2}=$ $\left[0,\left(T_{2}^{\prime}\right)^{-1}\right] P^{-1}$, where $T_{2}$ is any given $(n-k) \times(n-k)$ nonsingular matrix. ${ }^{21}$

Similarly, in the case that our problem is to maximize the modified objective (2.9) defined by a vector of initial multipliers $\tilde{\Theta}_{t_{0}-1} \equiv \tilde{\varphi}_{2, t_{0}}=\left[\begin{array}{ll}0 & I_{n-k}\end{array}\right] P^{-1} \hat{\varphi}_{1, t_{0}}$, the initial conditions can be expressed as

$$
V_{2} \hat{\varphi}_{1, t_{0}}=\Xi_{1, t_{0}-1}
$$

where $\Xi_{1, t_{0}-1} \equiv\left(T_{2}^{\prime}\right)^{-1} \tilde{\Theta}_{t_{0}-1}$ is merely a transformation of the initial multipliers.

Thus, when $q=0$, we can rewrite the first-order conditions (4.1)-(4.2) into a system involving the variables $\hat{\varphi}_{1, t}$ which consists of the conditions (4.8) that must hold for all $t \geq t_{0}$, conditions (4.10) that must hold for all $t>t_{0}$, and the initial condition (4.11).

Appendix A.8 considers the case in which $q>0$. As shown in that appendix, even if $q>0$, our optimal target criterion may also be constructed using solely the set of equations (4.8), (4.10) and (4.11), under some additional assumptions (i.e., Assumptions 5 and 6 listed there), where the

\footnotetext{
${ }^{21}$ In the case that $q=0, T_{2}$ may be any nonsingular matrix, and so we may as well set $T_{2}=I_{n-k}$. When $q>0, T_{2}$ must satisfy additional conditions, stated in Appendix A.8.
} 
matrix $V_{2}$ again depends on $P$, though in a more complicated fashion. Under those assumptions, the equilibrium relations (3.2) are decomposable into two sub-systems such that some of the endogenous variables are determined independently of any policy choices. We call such variables "inessential." This allows us to express the desired target criterion only as a function of the subset of $m-q$ "essential" target variables $\hat{\tau}_{1, t}$, regardless of the path of the "inessential" target variables $\hat{\tau}_{2, t}$.

\subsection{Implications of the Target Criterion in the Case of Unidimensional Policy}

We now specialize the problem by restricting our attention to the case in which there is a single dimension of policy variation each period, so that $p=m-n=1$. (Note that this case covers conventional analyses of monetary stabilization policy, where the single dimension of policy choice corresponds to a target for a short-term nominal interest rate or for the money supply each period.) In this case, there is only a single block of equations of the form (4.8), and we shall drop the $i$ subscript for the remainder of this section. The derivation of the optimal target criterion below applies both when $q=0$ and when $q>0$, provided that in the latter case, certain additional assumptions stated in Appendix A.8 also hold. In the general case, it is useful to define the quantities $\tilde{n} \equiv n-q>0, \tilde{m} \equiv m-q>0$. Furthermore, in the case that $q>0$, we define $k_{2}$ $\left(0 \leq k_{2} \leq q\right)$ as the number of generalized eigenvalues of the $q \times q$ matrix pencil $B_{2}^{\prime}-\mu J_{2}^{\prime}$ that are inside the unit circle. The number of "essential" predetermined state variables is then given by $k_{1} \equiv k-k_{2} \geq 0$. (In the case that $q=0$, we have $k_{1}=k$.)

We first state the restrictions on the process $\left\{\hat{\tau}_{1, t}\right\}$ that are required in order for there to exist a bounded process $\left\{\hat{\varphi}_{1, t}\right\}$ consistent with (4.8). (These conditions are in turn necessary, though not sufficient, for satisfaction of the complete set of linearized FOCs.) Let us first define the $\tilde{n} \times(\tilde{n}+1)$ matrix polynomial

$$
\Gamma(\mu) \equiv\left[\begin{array}{ccccc}
1 & 0 & \cdots & & 0 \\
(-\mu) & 1 & 0 & \cdots & 0 \\
\vdots & & \ddots & \ddots & \vdots \\
(-\mu)^{\tilde{n}-1} & \cdots & (-\mu) & 1 & 0
\end{array}\right]
$$

and the $\tilde{n}+1$-dimensional vector polynomial

$$
\delta(\mu)^{\prime} \equiv\left[\begin{array}{llll}
1 & (-\mu) & \cdots & (-\mu)^{\tilde{n}}
\end{array}\right]
$$

We can then define a moving average of the target variables

$$
z_{t} \equiv \delta\left(\beta^{-1} L\right)^{\prime} \hat{\tau}_{1, t}
$$


We can then establish the following useful result.

Lemma 4 Two processes $\left\{\hat{\tau}_{1, t}\right\}$ and $\left\{\hat{\varphi}_{1, t}\right\}$ specified for all $t \geq t_{0}$ satisfy (4.8) for all $t \geq t_{0}$ if and only if they satisfy

$$
\hat{\varphi}_{1, t}=\beta E_{t}\left[\Gamma\left(\beta L^{-1}\right) \hat{\tau}_{1, t}\right]
$$

and

$$
E_{t} z_{t+\tilde{n}}=0
$$

for all $t \geq t_{0}$, where the implied process $\left\{z_{t}\right\}$ is defined by (4.12).

The proof is in Appendix A.4. This result implies that a bounded process for the target variables $\left\{\hat{\tau}_{1, t}\right\}$ is consistent with condition (4.8) if and only if the evolution of $\left\{z_{t}\right\}$ satisfies (4.14); for given any bounded process satisfying (4.14), (4.13) defines a bounded process $\left\{\hat{\varphi}_{1, t}\right\}$ which will satisfy (4.8). It remains then only to ask what additional restrictions are required in order for the implied process $\left\{\hat{\varphi}_{1, t}\right\}$ to satisfy conditions (4.10) and (4.11) as well.

Condition (4.14) is thus an example of a target criterion that is necessary for policy to be optimal, but that is not in general sufficient for optimality; it is not in general a sufficiently restrictive condition to uniquely determine a forward path for the economy, either. Thus the target criterion that we seek must be stronger (except in special cases) than (4.14), but must imply (4.14). As it happens, the desired criterion can also be stated as a restriction upon the evolution of the scalar target variable $z_{t}$, albeit a stronger condition than (4.14). In order to state this criterion, it is useful to define a vector of revisions at date $t$ to the forecast path of the target variable,

$$
w_{t} \equiv\left[\begin{array}{c}
(-\beta)\left[z_{t}-E_{t-1} z_{t}\right] \\
(-\beta)^{2}\left[E_{t} z_{t+1}-E_{t-1} z_{t+1}\right] \\
\vdots \\
(-\beta)^{\tilde{n}}\left[E_{t} z_{t+\tilde{n}-1}-E_{t-1} z_{t+\tilde{n}-1}\right]
\end{array}\right] .
$$

It is also useful to define the matrix of current and past forecast revisions

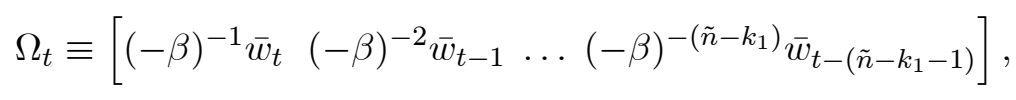

where $\bar{w}_{t}$ is the vector consisting of the first $k_{1}$ rows of $w_{t}$. (Note that $\Omega_{t}$ is a null matrix unless $0<k_{1}<\tilde{n}$; if $k_{1}=0, \Omega_{t}$ has no rows, while if $k_{1}=\tilde{n}$, it has no columns.)

The optimal target criterion can be expressed more simply if we assume one further regularity condition. Let the matrix $V_{2}$ be partitioned as

$$
V_{2} \equiv\left[\begin{array}{ll}
V_{21} & V_{22}
\end{array}\right]
$$


where $V_{21}$ is of dimension $\left(\tilde{n}-k_{1}\right) \times k_{1}$ and $V_{22}$ is of dimension $\left(\tilde{n}-k_{1}\right) \times\left(\tilde{n}-k_{1}\right)$. We can now state our final regularity condition.

Assumption 4 The submatrix $V_{22}$ is non-singular ( $\operatorname{det} V_{22} \neq 0$ ).

Note that for any $k_{1}<\tilde{n}$, this assumption holds for a generic non-singular matrix $P$. Moreover, when $k_{1}=0$ (there are no "essential" predetermined state variables), the assumption is necessarily satisfied, as then $V_{22}=V_{2}$, which is a full-rank matrix. The usefulness of this assumption is that it implies that any $\tilde{n}$-dimensional vector $v$ such that $V_{2} v=0$ can be reconstructed from its first $k_{1}$ elements.

We now state the optimal target criterion: this is the requirement that at each date $t \geq t_{0}$, the projected evolution of the process $\left\{z_{t}\right\}$ must be such that

$$
E_{t} z_{t+k_{1}}=(-\beta)^{-k_{1}} \operatorname{tr}\left[\Phi \Omega_{t}\right]
$$

where

$$
\Phi \equiv-V_{22}^{-1} V_{21}
$$

In evaluating this criterion in any period $t$, the references to forecasts at date $t-1$ and earlier are understood to refer to policy authority's historical forecasts, whether or not they were made on the basis of an expectation regarding policy in period $t$ and later that corresponds to current policy intentions. (In particular, forecasts dated $t_{0}-1$ may not be consistent with the policy regime that is adopted beginning in period $t_{0}$, but the target criterion refers to these historical forecasts nonetheless.) The set of paths for the target variables $\left\{\hat{\tau}_{1, t}\right\}$ from period $t_{0}$ onward that are consistent with the target criterion depends on a vector of initial conditions $e_{t_{0}-1}$, which includes the historical forecast revisions $\bar{w}_{t_{0}-j}$ for $1 \leq j \leq \tilde{n}-k_{1}-1$; the historical forecasts corresponding to the first $k_{1}$ rows of $\beta E_{t_{0}-1}\left[\Gamma\left(\beta L^{-1}\right) \hat{\tau}_{1, t_{0}}\right]$; and the historical path of the target variables $\hat{\tau}_{1, t_{0}-j}$ for $1 \leq j \leq \tilde{n}-k_{1}{ }^{22}$

Note that if $k_{1}<\tilde{n}$, satisfaction of (4.17) for all $t \geq t_{0}$ implies that

$$
\begin{aligned}
E_{t} z_{t+\tilde{n}} & =E_{t}\left[E_{t+\tilde{n}-k_{1}} z_{t+\tilde{n}}\right] \\
& =(-\beta)^{-k_{1}} \operatorname{tr}\left[\Phi E_{t} \Omega_{t+\tilde{n}-k_{1}}\right]=0
\end{aligned}
$$

\footnotetext{
${ }^{22}$ The vectors $\bar{w}_{t_{0}-j}$ are columns of $\Omega_{t_{0}}$ (and of $\Omega_{t}$ for the next few periods as well); the forecasts $\beta E_{t_{0}-1}\left[\Gamma\left(\beta L^{-1}\right) \hat{\tau}_{1, t_{0}}\right]$ are needed to define the forecast revisions $\bar{w}_{t_{0}}$, that also constitute a column of $\Omega_{t_{0}}$ and of subsequent $\Omega_{t}$; and the lagged variables $\hat{\tau}_{1, t_{0}-j}$ are needed to determine the value of $z_{t_{0}+k_{1}}$, as well as the value of $z_{t+k_{1}}$ for the next few periods, in addition to the evolution of the target variables from $t_{0}$ onward.
} 
for any $t \geq t_{0}$, using the fact that $E_{t} \bar{w}_{t+j}=0$ for any $t \geq t_{0}$ and any $j \geq 1$. But if $k_{1}=\tilde{n}, \Omega_{t}$ is a null matrix, and (4.17) also implies that $E_{t} z_{t+\tilde{n}}=0$ in this case as well. Hence the target criterion (4.17) implies condition (4.14), although (except when $k_{1}=\tilde{n}$ ) it is not implied by it. In fact, one can show that this stronger target criterion is also a necessary condition for optimality, at least in the case of initial pre-commitments of a particular sort. ${ }^{23}$

Proposition 2 Given Assumption 4 and any initial conditions $e_{t_{0}-1}$, there exists a vector of initial Lagrange multipliers $\Xi_{1, t_{0}-1}$, such that any processes $\left\{\hat{\tau}_{1, t}\right\}$ and $\left\{\hat{\varphi}_{1, t}\right\}$ for $t \geq t_{0}$ that satisfy conditions (4.8) for all $t \geq t_{0}$, (4.10) for all $t>t_{0}$, and the initial condition (4.11), must also satisfy the target criterion (4.17) for all $t \geq t_{0}$.

The proof of this proposition is given in Appendix A.5. To be more specific, the vector of initial Lagrange multipliers with the asserted property is

$$
\Xi_{1, t_{0}-1} \equiv V_{22} \chi_{t_{0}-1}+V_{2} \beta E_{t_{0}-1}\left[\Gamma\left(\beta L^{-1}\right) \hat{\tau}_{1, t_{0}}\right]
$$

where $\chi_{t-1}$ is the vector whose $j$ th element is equal to

$$
\chi_{t-1}^{j} \equiv(-\beta)^{k_{1}+j} E_{t-1} z_{t+k_{1}+j-1}-\sum_{i=1}^{\tilde{n}-k_{1}-j}(-\beta)^{-i} \phi_{j+i}^{\prime} \bar{w}_{t-i}
$$

using the notation $\phi_{j}^{\prime}$ for the $j$ th row of the matrix $\Phi$. Note that each element of $\Xi_{1, t_{0}-1}$ is a linear function of the vector of initial conditions $e_{t_{0}-1}$ defined above. Proposition 2 is established by showing that for this specification of the initial multipliers, the Lagrange multipliers defined by (4.13) satisfy conditions (4.10) and (4.11) if and only if the evolution of the target variables is consistent with (4.17) for each $t \geq t_{0}$.

The rule (4.18) for selection of initial Lagrange multipliers as a function of the initial condition $e_{t_{0}-1}$ is an example of a self-consistent rule, because of the following result.

Lemma 5 Suppose that Assumption 4 holds and let the bounded processes $\left\{\hat{\tau}_{1, t}\right\}$ and $\left\{\hat{\varphi}_{1, t}\right\}$ for $t \geq t_{0}$ satisfy conditions (4.8) for all $t \geq t_{0}$, (A.62) for all $t>t_{0}$, and the initial condition (A.64), where the initial Lagrange multipliers are given by (4.18). Then in each period $t \geq t_{0}$,

$$
V_{2} \hat{\varphi}_{1, t}=\Xi_{1, t-1},
$$

\footnotetext{
${ }^{23}$ In the case of more general specifications of the initial pre-commitments and/or the initial Lagrange multipliers $\Xi_{1, t_{0}-1}$, we can also show that it is necessary that (4.17) be satisfied for all $t$ after some finite date; but this extension of Proposition 4 is not necessary for the subsequent discussion, and hence is omitted.
} 
where

$$
\Xi_{1, t-1} \equiv V_{22} \chi_{t-1}+V_{2} \beta E_{t-1}\left[\Gamma\left(\beta L^{-1}\right) \hat{\tau}_{1, t}\right]
$$

The proof is in Appendix A.6. Since conditions (4.11) and (4.18) are just specializations to period $t_{0}$ of the more general relations (4.20) and (4.21), it follows that the proposed rule for assigning the initial Lagrange multipliers is self-consistent. Because of Proposition 4(c), this assignment of the initial Lagrange multipliers as a function of the initial condition corresponds to a rule for assigning initial pre-commitments $\bar{g}_{t_{0}}$ as a function of the initial condition (and the economy's state in period $t_{0}$ ), that will also represent a self-consistent rule, in the sense defined earlier.

Proposition 2 implies that fulfillment of the target criterion is a necessary condition for fulfillment of the FOCs, and hence a necessary condition for optimality, in the case of a problem involving initial pre-commitments of the kind just discussed. But consistency of the paths of the target variables with the target criterion (4.17) is also sufficient for satisfaction of the FOCs, as established in the proof of the following proposition. Hence the target criterion (4.17) determines a unique bounded solution for the equilibrium evolution of the endogenous variables, and by Proposition 1(b), the evolution determined by this criterion represents a local linear approximation to the optimal evolution, under the modified problem defined by initial Lagrange multipliers (4.18). We thus obtain our main conclusion.

\section{Proposition 3 Suppose that Assumptions 1-4 are satisfied. ${ }^{24}$}

(a) Then the requirements that the evolution of the endogenous variables $\left\{\tilde{y}_{t}\right\}$ for $t \geq t_{0}$ be consistent with the target criterion (4.17) for all $t \geq t_{0}$, and satisfy the bound $\|\tilde{y}\|<\infty$, where the norm is defined as in (3.7), determine a unique solution for the path of these variables, given any initial conditions $e_{t_{0}-1}$.

(b) Moreover, the unique solution determined by these criteria represents a local linear approximation to the evolution $\left\{y_{t}\right\}$ that maximizes the modified objective function (2.9), in the case of initial conditions close enough to consistency with the steady state and disturbance processes under which $\xi_{t}$ remains always close enough to $\bar{\xi}$, if the initial Lagrange multipliers $\Theta_{t_{0}-1}$ are given by

$$
\Theta_{t_{0}-1}=\bar{\Theta}+T_{2}^{\prime} \Xi_{t_{0}-1}
$$

where the multipliers $\Xi_{1, t_{0}-1}$ are given by (4.18), and the multipliers $\Xi_{2, t_{0}-1}$ (if this is not a null vector) may be chosen arbitrarily.

\footnotetext{
${ }^{24}$ In the case that $q>0$, we need also to assume that Assumptions 5 and 6, stated in Appendix A.8, are satisfied.
} 
(c) The rule (4.18) for the selection of the initial multipliers is self-consistent, in the sense that (4.21) holds for all $t \geq t_{0}$ in the linear approximation to the solution to the above problem. Hence the equilibrium determined by the target criterion (4.17) is optimal from a timeless perspective.

The proof is in Appendix A.7.

\section{Application: Optimal Policy in an Exact New Keynesian Model}

To illustrate how the proposed optimal target criterion can be derived in a nonlinear model in which the objective of policy is assumed to be the maximization of household utility, we consider the exact New Keynesian model presented in Benigno and Woodford (2005). While this very stylized model abstracts from a number of the frictions often included in larger-scale models used for empirical analysis or for practical policy recommendations, its relative simplicity allows us to illustrate clearly how to derive the optimal target criterion in a fully analytical fashion, and provides intuition about the proposed target criterion.

\section{$5.1 \quad$ Model}

We now provide a very succinct description of the model, leaving the details to Appendix C. In this model, each household seeks to maximize its lifetime utility which depends in a time-separable fashion on consumption of an aggregate of all goods and (negatively) on the amount of labor supplied. Each differentiated good is supplied by a single monopolistically competitive producer who uses labor as the only variable input. As Benigno and Woodford (2005) show, the utility of the representative household, which is also the policymaker's welfare objective function, can be expressed in the form (2.1) where the period $t$ utility can be written as

$$
\pi\left(y_{t}, \xi_{t}\right)=U\left(Y_{t}, \Delta_{t} ; \xi_{t}\right) \equiv u\left(Y_{t} ; \xi_{t}\right)-v\left(Y_{t} ; \xi_{t}\right) \Delta_{t}
$$

where $Y_{t}$ is aggregate production of the composite good, $\Delta_{t}$ is a measure of price dispersion, and $\xi_{t}$ is a vector of exogenous variables (including shocks to technology, preferences, and fiscal policy). Here both the utility from consumption and the disutility of labor supply have been expressed as functions of the total quantity produced; the disutility of labor supply also depends on price dispersion, as this affects the composition of output and hence the labor required to produce a given amount of the composite good. 
The producers are wage takers on the labor market and choose their prices to maximize the present discounted value of future profits. As in Calvo's (1983) model of staggered pricing, we assume that producers fix the prices of their goods for a random interval of time, with a constant fraction $\alpha \in[0,1)$ of prices remaining unchanged in any given period. Aggregating the producers' optimal pricing decisions yields a short-run aggregate supply relation between inflation and output of the form

$$
\frac{1-\alpha \Pi_{t}^{\theta-1}}{1-\alpha}=\left(\frac{H_{t}}{K_{t}}\right)^{\frac{\theta-1}{1+\omega \theta}}
$$

where $\Pi_{t} \equiv P_{t} / P_{t-1}$ is the gross inflation rate and the variables $H_{t}$ and $K_{t}$ are given by the recursive expressions

$$
\begin{aligned}
H_{t} & =\left(1-\varsigma_{t}\right) \cdot u_{Y}\left(Y_{t}, \xi_{t}\right) \cdot Y_{t}+\alpha \beta E_{t}\left[\Pi_{t+1}^{\theta-1} H_{t+1}\right] \\
K_{t} & =\frac{\theta \mu_{t}^{w}}{\theta-1} \cdot v_{Y}\left(Y_{t}, \xi_{t}\right) \cdot Y_{t}+\alpha \beta E_{t}\left[\Pi_{t+1}^{\theta(1+\omega)} K_{t+1}\right] .
\end{aligned}
$$

Here $\varsigma_{t} \in[0,1)$ is an exogenous sales tax rate; $\mu_{t}^{w} \geq 1$ is an exogenous wage markup factor; $\theta>1$ is the elasticity of substitution across individual goods; and $\omega \geq 0$ is the elasticity of the function $v_{Y}$ with respect to increases in output. The law of motion of the measure of price dispersion is further given by

$$
\Delta_{t}=\alpha \Delta_{t-1} \Pi_{t}^{\theta(1+\omega)}+(1-\alpha)\left(\frac{1-\alpha \Pi_{t}^{\theta-1}}{1-\alpha}\right)^{\frac{\theta(1+\omega)}{\theta-1}} .
$$

While the optimal intertemporal allocation of households' expenditures determines period- $t$ output as a function of expectations of future output, inflation and the nominal interest rate, this doesn't constitute a constraint on the policy problem, as the central bank can always choose a nominal interest rate that satisfies this equation. As a result, the only relevant constraints facing the policymaker are given by (5.1)-(5.4), describing the necessary connections between the evolution of aggregate real activity, inflation, and price dispersion. Using (5.1) to substitute for the variable $\Pi_{t}$, we can express the resulting restrictions by a system of the form $(2.2)-(2.3)$, where the vector of endogenous variables is given by $y_{t} \equiv\left[Y_{t}, \Delta_{t}, H_{t}, K_{t}\right]^{\prime}, F$ is a single function, and $g$ is a pair of functions. We thus have $m=4$ endogenous variables, $n=3$ equilibrium restrictions per period, and $k=1$ of these restrictions involves only contemporaneous or past variables. It is also useful to note that in this model, the functions $g$ take the special form

$$
g\left(y_{t}, \xi_{t} ; y_{t+1}\right)=\check{g}\left(Y_{t}, \xi_{t}\right)-Z_{t}+\alpha \beta \Phi\left(Z_{t+1}\right)
$$

where $Z_{t} \equiv\left[H_{t}, K_{t}\right]^{\prime}$ is a subset of the endogenous variables, and $\check{g}$ and $\Phi$ are vectors of nonlinear functions, each with two elements. 


\subsection{Characterizing the Optimal Target Criterion}

By computing the nonlinear FOCs that characterize optimal policy, we show in Appendix C that an optimal steady state exists, in which the price level remains stable so that $\Pi_{t}=\bar{\Pi}=1$ and price dispersion is zero $\left(\Delta_{t}=\bar{\Delta}=1\right)$. We then log-linearize the nonlinear structural equations and FOCs around these steady-state values of the endogenous variables, and compute the canonical decomposition of the matrix pencil $\bar{A}-\mu \bar{I}$. This allows us to compute our target criterion.

We show in Appendix $\mathrm{C}$ that the linearized structural equations have a bounded solution (and hence Assumption 1 is satisfied) provided that either the degree of inefficiency of steady-state output is not too great, or the share of output consumed by the government is not too large. It is easy to verify in addition that the pencil $\bar{A}-\mu \bar{I}$ satisfies Assumption 2 . We show in the Appendix that the minimal degree associated with $\bar{A}-\mu \bar{I}$ is $\tilde{n}=2$. It then follows from Lemma 3 that there exist nonsingular matrices $P$ and $Q$ of dimensions $3 \times 3$ and $4 \times 4$ respectively that satisfy

$$
(\bar{A}-\mu \bar{I})^{\prime}=Q\left[\begin{array}{cc}
M(\mu) & 0 \\
0 & B_{2}^{\prime}-\mu J_{2}^{\prime}
\end{array}\right] P
$$

where $M(\mu)$ is the $(\tilde{n}+1) \times \tilde{n}$ matrix pencil

$$
M(\mu)=\left[\begin{array}{ll}
\mu & 0 \\
1 & \mu \\
0 & 1
\end{array}\right]
$$

and $B_{2}^{\prime}-\mu J_{2}^{\prime}$ is a matrix pencil of dimension $1 \times 1$, with $B_{2}=\alpha<1$, and $J_{2}^{\prime}=1$. A second-order Taylor expansion of the Lagrangian can also be used to show that Assumption 3 is satisfied in this example. This calculation is explained in Benigno and Woodford (2012).

Given the dimension of $J_{2}, B_{2}$, and the fact that $\left|B_{2}\right|<1$, it follows that $q=k_{2}=1$, so that the model has one "inessential" variable, i.e., one variable whose linear dynamics are not affected by the policy choices. Interestingly, while the model involves one predetermined endogenous variable, namely the price dispersion measure $\Delta_{t}$, this variable turns out to be inessential in the model's linearized dynamics, though policy policy has second-order effects on the price dispersion in the exact model. Hence, while $k=1$ in the model, the number of predetermined variables when the system of equation is written only in terms of "essential" variables is $k_{1}=0$. Because $k_{1}=0, V_{22}$ is simply the inverse of the block of $P$ corresponding to the two "essential" forward-looking structural equations, and hence Assumption 4 is necessarily satisfied. ${ }^{25}$

\footnotetext{
${ }^{25}$ Section C.5 also shows that the additional assumptions required for the validity of Proposition 3 when $q>0$ are
} 
Since $k_{1}=0$, the general optimal target criterion (4.17) reduces to

$$
z_{t}=0
$$

Given the definition of the variable $z_{t}$ in (4.12), the optimal target criterion is backward-looking

$$
0=\left[\begin{array}{lll}
1 & -\beta^{-1} L & \beta^{-2} L^{2}
\end{array}\right] \hat{\tau}_{1 t}
$$

that is, a moving average of the current and past essential target gaps

$$
\hat{\tau}_{1 t}=\left[\begin{array}{ll}
I_{m-q} & 0
\end{array}\right] Q^{-1}\left(\tau_{t}-\tau_{t}^{*}\right)
$$

To obtain the target gaps $\tau_{t}-\tau_{t}^{*}$, we evaluate the second derivatives of $F(), g()$ and the objective function $\pi()$ at the steady state. As shown in Appendix C, the optimal target criterion takes the form

$$
(1-\alpha L)\left[\pi_{t}+\phi\left(x_{t}-x_{t-1}\right)\right]=0
$$

where $x_{t} \equiv \log \left(Y_{t} / Y_{t}^{*}\right)$ is an "output gap" relative to a target level of output; $Y_{t}^{*}$ is a function of the exogenous disturbances implicitly defined by the equation

$$
U_{Y}\left(Y_{t}^{*}, 1 ; \xi_{t}\right)+\bar{\Theta}^{\prime} \check{g}_{Y}\left(Y_{t}^{*}, \xi_{t}\right)=0
$$

where $\bar{\Theta}$ is the steady-state vector of Lagrange multipliers associated with the forward-looking constraints. In the case that the flexible-price steady-state level of output is efficient, ${ }^{26}$ then the zero-inflation steady state level of output $\bar{Y}$ is efficient, and $\bar{\Theta}=0$. In this special case, $Y_{t}^{*}$ is simply the utility-maximizing level of output given technology, preferences and government purchases at any point in time (defined by the FOC $U_{Y}\left(Y_{t}^{*}, 1 ; \xi_{t}\right)=0$ ). It also corresponds in this case to the flexible-price equilibrium level of output, if the distortion factors $\varsigma_{t}$ and $\mu_{t}^{w}$ are set equal to their steady-state values. More generally, $Y_{t}^{*}$ is not quite the same as either the efficient level of output or the flexible-price equilibrium level of output, but is closely related to both of them.

Condition (5.5) requires that eventually

$$
\pi_{t}+\phi\left(x_{t}-x_{t-1}\right)=0
$$

satisfied.

${ }^{26}$ This would in general require a negative steady-state sales tax $\varsigma$, to offset the distortion due to market power on the part of the monopolistically competitive producers. See Woodford (2003, chap. 6) for further discussion of this case. 
each period. ${ }^{27}$ Thus optimal policy can be characterized as a form of "flexible inflation targeting," in which deviations of the inflation rate from its optimal long-run value (zero) must at all times be in proportion to projected changes in the output gap. In Appendix C, we characterize the optimal value of the coefficient of proportionality $\phi$ in terms of model parameters. Unless both the degree of inefficiency of steady-state output and the share of output consumed by the government are large, $\phi$ will be a positive coefficient. In this case, the target criterion has the same form as equation (1.1) implied by the kind of linear-quadratic policy problem analyzed by authors such as Clarida et al. (1999). But here, both the definition of the "output gap" and the weight $\phi$ to be put on changes in the output gap are derived from the micro-foundations of the model, rather than an ad hoc quadratic objective function for the central bank; and the optimal target criterion involves additional transitory dynamics.

\section{Concluding Remarks}

We have considered a general class of nonlinear rational-expectations models in which policymakers seek to maximize an objective function. We have proposed a procedure to derive a target criterion that is: (i) consistent with the model's structural equations, (ii) strong enough to imply a unique equilibrium, and (iii) optimal, in the sense that a commitment to adjust the policy instrument at all dates so as to satisfy the target criterion maximizes the objective function.

Some general remarks are possible on the form of the optimal target criterion (4.17). We have established quite generally that there is a single composite target variable $z_{t}$ such that the target criterion can be expressed purely in terms of projections for the evolution of this variable. (This depends, however, on our assumption that policy can vary equilibrium outcomes along only one dimension per period.)

In general, the target criterion (4.17) has both backward-looking and forward-looking elements. The degree to which it has one character or the other depends on the degree to which the structural equations of the model are backward- or forward-looking. When the model's constraints are purely

\footnotetext{
${ }^{27}$ In the case of small initial price dispersion, $\hat{\Delta}_{t_{0}-1}=\mathcal{O}\left(\epsilon^{2}\right)$, as assumed in Benigno and Woodford (2005), it is possible to choose the initial lagged Lagrange multipliers in a self-consistent way and obtain a modified policy problem under which optimal policy will satisfy (5.7) from period $t_{0}$ onward. However, that method cannot be extended to deal with non-trivial levels of initial price dispersion (of order $\mathcal{O}(\epsilon)$ ) without requiring transitory departures of the left-hand side of (5.7) from its long-run value for most initial conditions, as implied by the target criterion derived here.
} 
forward-looking - or more precisely, when the reduced system of equations written in terms of the "essential" state variables contains no backward-looking structural relations (A.57), so that $k_{1}=0$ — the criterion (4.17) reduces to

$$
z_{t}=0
$$

In this case the optimal target criterion is necessarily purely backward-looking, i.e., it is a linear relation between current and past values of the target variables $\hat{\tau}_{1, t}$. This is the case illustrated by the example in section 4 .

If, instead (as is more generally the case), lagged variables enter the structural equations, the optimal target criterion involves forecasts as well, for a finite number of periods into the future. In the opposite polar case in which the model's constraints are purely backward-looking (i.e., they do not involve expectations) - or at least this is true when the model is written in terms of the essential variables only, so that $k_{1}=\tilde{n}$ - then the criterion (4.17) takes the form

$$
E_{t} z_{t+\tilde{n}}=0
$$

Since $z_{t+\tilde{n}}$ involves elements of $\hat{\tau}_{1, t+j}$ for $0 \leq j \leq \tilde{n}$, in this case the optimal target criterion is purely forward-looking, in the sense that it involves only the projected paths of the target variables $\hat{\tau}_{1, t}$ in current and future periods. In the intermediate cases, the target criterion involves both forecasts of target variables at least one period in the future and at least one lag of the target variables (as well as, in general, forecasts from one or more prior periods). The number of periods that the criterion requires one to look into the future is greater the larger is $k_{1}$ (and in this sense, the more backward-looking the model dynamics), and the number of periods from which past values remain relevant to the target criterion is greater the greater is $\tilde{n}-k_{1}$ (and in this sense, the more forward-looking the model dynamics).

While we have noted that the target criterion can quite generally be stated in terms of projections for a single composite target variable $z_{t}$, this variable will generally involve the paths (with differing lags) of all of the "essential" state variables $y_{1, t}^{*}$. However, there is a special case in which the target criterion need only involve the projections for a small number of economically meaningful variables, regardless of the complexity of the structural model. This is the case in which the objective function (2.1) is purely quadratic, as assumed in the hypotheses of Lemma 2. While this is unlikely to be true in a microfounded model in which the objective of policy is taken to be the maximization of household expected utility, central banks are often interested in policies that are optimal from the point of view of some quadratic loss function involving a small number of targets, 
though a very complex model of the economy may be used to evaluate how well alternative policies achieve this objective. In the case that Lemma 2 applies, the composite target variable $z_{t}$ is a linear function of the paths of the $m_{\tau}$ target variables $q_{t}-q_{t}^{*}$ that appear in the quadratic objective (4.3).

The procedure to derive an optimal target criterion has been illustrated in the context of a simple nonlinear model, but it can be readily derived in larger empirical models using existing numerical techniques. The optimal target criterion has been derived here in the context of models in which policy can vary equilibrium outcomes along only one dimension per period $(p=1)$. The setup proposed can however accommodate cases in which policy can vary the equilibrium along multiple dimensions. In such cases, there would be $p>1$ target criteria to satisfy each period. We leave the detailed analysis of this general case for future work.

Acknowledgement. We would like to thank Pierpaolo Benigno and Lars Svensson for helpful discussions, and the NSF for support of the authors' research under grants SES-0518770 and SES0820438. 


\section{References}

[1] Anderson, B.D.O., Moore, J.B., 1979. Optimal Filtering, Prentice Hall, Englewood Cliffs, NJ.

[2] Backus, D., Driffill, J., 1986. The consistency of optimal policy in stochastic rational expectations models. CEPR Disc. Paper no. 124.

[3] Benigno, P., Woodford, M., 2004. Optimal monetary and fiscal policy: a linear-quadratic approach, in: Gertler, M., Rogoff, K. (Eds.), NBER Macroeconomics Annual 2003, MIT Press, vol. 18, 271-333.

[4] Benigno, P., Woodford, M., 2005. Inflation stabilization and welfare: the case of a distorted steady state. J. Eur. Econ. Assocn. 3, 1185-1236. DOI: 10.1162/154247605775012914.

[5] Benigno, P., Woodford, M., 2012. Linear-quadratic approximation of optimal policy problems. J. Econ. Theory. 147(1), 1-42. DOI: 10.1016/j.jet.2011.10.012.

[6] Calvo, G., 1983. Staggered prices in a utility-maximizing framework. J. Monetary Econ. 12(3), 383-398. DOI: 10.1016/0304-3932(83)90060-0.

[7] Clarida, R., Galí, J., Gertler, M., 1999. The science of monetary policy: a new Keynesian perspective. J. Econ. Lit. 37(4), 1661-1707. DOI: 10.1257/jel.37.4.1661.

[8] Currie, D., Levine, P., 1993. Rules, Reputation and Macroeconomic Policy Coordination, Cambridge University Press, Cambridge.

[9] Gantmacher, F.R., 1959. The Theory of Matrices, vol. 2, AMS Chelsea Publishing, Providence, Rhode Island.

[10] Giannoni, M.P., Woodford, M., 2003a. Optimal interest-rate rules: I. general theory," NBER working paper no. 9419 .

[11] Giannoni, M.P., Woodford, M., 2003b. How forward-looking is optimal monetary policy? J. Money Credit Bank. 35(6): 1425-1469. URL: http://www.jstor.org/stable/3649892.

[12] Giannoni, M.P., Woodford, M., 2005. Optimal inflation targeting rules, in: Bernanke, B.S., Woodford, M. (Eds.), The Inflation Targeting Debate, University of Chicago Press, Chicago.

[13] Hansen, L.P., Sargent, T.J., 2013. Recursive Models of Dynamic Linear Economies. Princeton University Press, Princeton. 
[14] Khan, A., King, R.G., Wolman, A.L., 2003. Optimal monetary policy. Rev. Econ. Stud. 70(4): 825-860. DOI: 10.1111/1467-937X.00269.

[15] Marcet, A., Marimon, R., 1998. Recursive Contracts. Universitat Pompeu Fabra working paper no. 337 .

[16] Pappas, T., Laub. A.J., Sandell, N.R., 1980. On the numerical solution of the discretetime algebraic Riccati equation. IEEE Trans. Autom. Control. AC25(4), 631-641. DOI: 10.1109/TAC.1980.1102434.

[17] Svensson, L.E.O., 1997. Inflation forecast targeting: implementing and monitoring inflation targeting," Eur. Econ. Rev. 41(6), 1111-1146. DOI: 10.1016/S0014-2921(96)00055-4.

[18] Svensson, L.E.O., 2003. What is wrong with Taylor rules? Using judgment in monetary policy through targeting rules. J. Econ. Lit. 41(2), 426-477. DOI: 10.1257/002205103765762734.

[19] Svensson, L.E.O., 2005. Monetary policy with judgment: forecast targeting. Int. J. Cent. Bank. $1,1-54$.

[20] Svensson, L.E.O., 2011. Inflation targeting, in: Friedman, B.M., Woodford, M. (Eds.), Handbook of Monetary Economics, Elsevier, vol. 3, chap. 22, 1237-1302. DOI: 10.1016/B978-0-44453454-5.00010-4.

[21] Svensson, L.E.O., Woodford, M., 2005. Implementing optimal policy through inflation-forecast targeting," in: Bernanke, B.S., Woodford, M. (Eds.), The Inflation Targeting Debate, University of Chicago Press, Chicago.

[22] Woodford, M., 1999. Commentary: how should monetary policy be conducted in an era of price stability? in: New Challenges for Monetary Policy, Federal Reserve Bank of Kansas City, Kansas City.

[23] Woodford, M., 2003. Interest and Prices: Foundations of a Theory of Monetary Policy, Princeton University Press, Princeton.

[24] Woodford, M., 2011. Optimal monetary stabilization policy, in: Friedman, B.M., Woodford, M. (Eds.), Handbook of Monetary Economics, Elsevier, vol. 3, chap. 14, 723-828. DOI: 10.1016/B978-0-444-53454-5.00002-5. 
[25] Woodford, M., 2012. Forecast targeting as a monetary policy strategy: policy rules in practice, in: Koenig, E.F., Leeson, R., Taylor, J.B. (Eds.), The Taylor Rule and the Transformation of Monetary Policy, Hoover Institution Press, Palo Alto. 


\section{A Proof of Lemmas and Propositions}

\section{A.1 Proof of Proposition 1}

Here we show that there exists a unique bounded solution to the system of equations consisting of the linearized structural equations (3.2)-(3.4) together with the linearized FOCs (3.5)-(3.6). If we adjoin to these equations the identities

$$
\begin{aligned}
\tilde{y}_{t} & =\tilde{y}_{t}, \\
E_{t} \tilde{\varphi}_{t+1} & =E_{t} \tilde{\varphi}_{t+1},
\end{aligned}
$$

then the system consisting of (3.2), (3.5), and (A.1)-(A.2) can be rewritten in matrix form as

$$
\bar{M} E_{t} d_{t+1}=\bar{N} d_{t}-\bar{N}_{s} \bar{s}_{t}
$$

where $d_{t}$ is the $2(m+n)$-dimensional vector

$$
d_{t} \equiv\left[\begin{array}{c}
\tilde{\varphi}_{t} \\
\tilde{y}_{t-1} \\
E_{t} \tilde{\varphi}_{t+1} \\
\tilde{y}_{t},
\end{array}\right]
$$

$\bar{s}_{t}$ is a vector of exogenous disturbances that includes the elements of both $\tilde{\xi}_{t}$ and $\tilde{\xi}_{t-1}$, and

$$
\bar{M} \equiv\left[\begin{array}{cc}
\bar{M}_{11} & \bar{M}_{12} \\
I_{m+n} & 0
\end{array}\right], \quad \bar{N} \equiv\left[\begin{array}{cc}
-\beta^{-1} \bar{M}_{12}^{\prime} & 0 \\
0 & I_{m+n}
\end{array}\right]
$$

where

$$
\bar{M}_{11} \equiv\left[\begin{array}{cc}
0 & \bar{A} \\
\bar{A}^{\prime} & S
\end{array}\right]=\bar{M}_{11}^{\prime}, \quad \text { and } \quad \bar{M}_{12} \equiv\left[\begin{array}{cc}
0 & -\bar{I} \\
0 & \beta R^{\prime}
\end{array}\right]
$$

Here we use the fact that $S$ is symmetric to obtain $\bar{M}_{11}=\bar{M}_{11}^{\prime}$.

In addition to conditions (A.3), the process $\left\{d_{t}\right\}$ must satisfy (3.3) and (3.6), and thus

$$
F_{d}\left[d_{t}-E_{t-1} d_{t}\right]=F_{s}\left[\bar{s}_{t}-E_{t-1} \bar{s}_{t}\right]
$$

for all $t>t_{0}$, where $F_{d}$ is the $(m+n) \times 2(m+n)$ matrix

$$
F_{d} \equiv\left[\begin{array}{cccc}
S_{2} & 0 & 0 & 0 \\
0 & 0 & 0 & \bar{I}_{1} \\
0 & I_{m} & 0 & 0
\end{array}\right],
$$

using the notation

$$
S_{2} \equiv\left[0 I_{n-k}\right]
$$

for the $(n-k) \times n$ matrix that selects the last $n-k$ elements of any $n$-vector. (The first $n-k$ rows correspond to conditions (3.6), the next $k$ rows correspond to conditions (3.3), and the final $m$ rows state that the elements of $\tilde{y}_{t-1}$ cannot be affected by surprises in period $t$.)

In period $t_{0}$, the process must satisfy (3.4) and hence

$$
F_{d} d_{t_{0}}=f_{t_{0}}
$$


where $f_{t_{0}}$ is a vector of $m+n$ initial conditions

$$
f_{t_{0}} \equiv\left[\begin{array}{c}
\tilde{\Theta}_{t_{0}-1} \\
\bar{A}_{1} \tilde{y}_{t_{0}-1}+\bar{D} \tilde{\xi}_{t_{0}}-\beta \bar{F}_{t_{0}} \\
\tilde{y}_{t_{0}-1}
\end{array}\right],
$$

all of which are either predetermined or exogenous.

The following lemmas establish useful properties of the matrix pencil $\bar{M}-\mu \bar{N}$.

Lemma 6 Given Assumptions 2(b) and 3, the matrix pencil $\bar{M}-\mu \bar{N}$ is regular; that is, its determinant is non-zero for at least some complex $\mu$.

Proof. The determinant of $\bar{M}-\mu \bar{N}$ can be expressed as follows:

$$
\begin{aligned}
\operatorname{det}(\bar{M}-\mu \bar{N}) & =\operatorname{det}\left[\begin{array}{cc}
\bar{M}_{11}+\mu \beta^{-1} \bar{M}_{12}^{\prime} & \bar{M}_{12} \\
I & -\mu I
\end{array}\right] \\
& =\operatorname{det}(-\mu I) \cdot \operatorname{det}\left[\left(\bar{M}_{11}+\mu \beta^{-1} \bar{M}_{12}^{\prime}\right)-\bar{M}_{12}(-\mu I)^{-1}\right] \\
& =(-\mu)^{n+m} \cdot \operatorname{det}\left[\bar{M}_{11}+\mu \beta^{-1} \bar{M}_{12}^{\prime}+\mu^{-1} \bar{M}_{12}\right] \\
& =(-\mu)^{n+m} \cdot \operatorname{det}\left[\begin{array}{cc}
0 & \bar{A}-\mu^{-1} \bar{I} \\
\bar{A}^{\prime}-\mu \beta^{-1} \bar{I}^{\prime} & S+\mu^{-1} \beta R^{\prime}+\mu R
\end{array}\right] .
\end{aligned}
$$

The matrix pencil $\bar{M}-\mu \bar{N}$ is regular provided that its determinant is non-zero for at least some complex $\mu$.

Suppose the determinant is instead zero for all $\mu$. This means that there must exist finite-order vector polynomials $(\varphi(\mu), y(\mu))$ such that

$$
\left[\begin{array}{cc}
0 & \bar{A}-\mu^{-1} \bar{I} \\
\bar{A}^{\prime}-\mu \beta^{-1} \bar{I}^{\prime} & S+\mu^{-1} \beta R^{\prime}+\mu R
\end{array}\right]\left[\begin{array}{l}
\varphi(\mu) \\
y(\mu)
\end{array}\right]=0
$$

for all $\mu \neq 0$, and $(\varphi(\mu), y(\mu))$ are not both equal to zero for all $\mu$. In addition, the solution cannot involve $y(\mu)=0$. For if there exists a function $\varphi(\mu) \equiv \sum_{i=0}^{k} \varphi_{i} \mu^{i}$ satisfying (A.8) with $y(\mu)=0$, one must have $\left[\bar{A}^{\prime}-\mu \beta^{-1} \bar{I}^{\prime}\right] \varphi(\mu)=0$. But this would imply that the function $\varphi(\mu) \equiv$ $\sum_{i=0}^{k} \varphi_{k-i} \mu^{i}$ must satisfy (3.9), violating Assumption 2(b). Hence we must have $y(\mu) \neq 0$. Writing $y(\mu) \equiv \sum_{i=0}^{\infty} y_{i} \mu^{i}$ (where all but a finite number of the $y_{i}$ are zero), the first line of (A.8) implies that the sequence $\left\{y_{i}\right\}$ satisfies the hypotheses of Assumption 3. The second line implies that

$$
y\left(\mu^{-1} \beta\right)^{\prime}\left[S+\mu^{-1} \beta R^{\prime}+\mu R\right] y(\mu)=0
$$

for all $\mu$. Writing this expression in the form $\sum_{j=-(k+1)}^{k+1} \gamma_{j} \mu^{j}$, where $k$ is the order of $y(\mu)$, it follows that we must have $\gamma_{j}=0$ for all $j$. In particular, we must have $\gamma_{0}=0$. But $\gamma_{0}$ is just the left-hand side of (3.12), so this violates Assumption 3. It follows that $\operatorname{det}(\bar{M}-\mu \bar{N})$ cannot be zero for all $\mu$.

It is then possible to factor the polynomial $\operatorname{det}[\lambda \bar{M}-\rho \bar{N}]$ as

$$
\prod_{i=1}^{2(n+m)}\left(\alpha_{i} \lambda-\beta_{i} \rho\right)
$$

where for any $i$, the complex numbers $\alpha_{i}$ and $\beta_{i}$ are not both equal to zero. Let $s$ be the number of factors for which $\alpha_{i} \neq 0$ and $\left|\beta_{i} / \alpha_{i}\right|<1$. There must then be $2 m+2 n-s$ factors for which $\beta_{i} \neq 0$ and $\left|\alpha_{i} / \beta_{i}\right| \leq 1$.

This implies that the matrices $\bar{M}$ and $\bar{N}$ can be decomposed as stated in the following lemma. 
Lemma 7 Given Assumptions 2(b) and 3, there must exist non-singular $2(m+n) \times 2(m+n)$ real matrices $\bar{U}, \bar{V}$ such that

$$
\bar{U} \bar{M} \bar{V}=\left[\begin{array}{cc}
I_{s} & 0 \\
0 & \Omega
\end{array}\right], \quad \bar{U} \bar{N} \bar{V}=\left[\begin{array}{cc}
\Lambda^{\prime} & 0 \\
0 & I_{2 n+2 m-s}
\end{array}\right] .
$$

Here $\Omega$ is a $(2 m+2 n-s) \times(2 m+2 n-s)$ real matrix for which all eigenvalues have modulus less than or equal to 1 while $\Lambda$ is an $s \times s$ real matrix for which all eigenvalues have modulus less than 1 .

Proof. Under Assumptions 2(b) and 3, Lemma 6 implies that $\bar{M}-\mu \bar{N}$ is a real regular matrix pencil of dimensions $(2 m+2 n) \times(2 m+2 n)$. It follows from Theorem 3 of Gantmacher (1959, Chap. 12), or its version for a real canonical form proved in Appendix B.3, that there exist real invertible matrices $\tilde{U}, \bar{V}$ of dimensions $(2 m+2 n) \times(2 m+2 n)$ such that

$$
\tilde{U} \bar{M} \bar{V}=\left[\begin{array}{cc}
I & 0 \\
0 & \tilde{G}
\end{array}\right], \quad \tilde{U} \bar{N} \bar{V}=\left[\begin{array}{cc}
\tilde{H} & 0 \\
0 & I
\end{array}\right]
$$

where $\tilde{G}$ is an invertible matrix of the real Jordan form and $\tilde{H}$ is a real nilpotent matrix of the Jordan form.

Let us factor the polynomial $\operatorname{det}[\lambda \bar{M}-\rho \bar{N}]$ as in (A.9) and let $\nu(0 \leq \nu \leq 2 m+2 n)$ be the number of factors $\left(\alpha_{i} \lambda-\beta_{i} \rho\right)$ for which the complex numbers $\beta_{i}=0$, while the numbers $\alpha_{i}$ are necessarily nonzero. (Note that since the eigenvalues of $\bar{M}-\mu \bar{N}$ are the quantities $\alpha_{i} / \beta_{i}$, these $\nu$ factors correspond to the $\nu$ "infinite" eigenvalues of $\bar{M}-\mu \bar{N}$.) The existence of a decomposition of the form (A.11) implies that the factors of the characteristic polynomial $\operatorname{det}[\lambda \bar{M}-\rho \bar{N}]$ in (A.9) are the same as those of

$$
\operatorname{det}[\lambda I-\rho \tilde{H}] \cdot \operatorname{det}[\lambda \tilde{G}-\rho I] \text {. }
$$

Since $\tilde{H}$ is nilpotent, $\operatorname{det}[\lambda I-\rho \tilde{H}]$ must correspond to the $\nu$ factors for which the $\beta_{i}=0$ and $\alpha_{i} \neq 0$. The matrix pencil $\lambda I-\rho \tilde{H}$ is thus of dimensions $\nu \times \nu$. This implies that the matrix pencil $\lambda \tilde{G}-\rho I$ is of dimensions $(2 m+2 n-\nu) \times(2 m+2 n-\nu)$ and its determinant is the product of the $2 m+2 n-\nu$ factors $\left(\alpha_{i} \lambda-\beta_{i} \rho\right)$ for which the complex numbers $\beta_{i} \neq 0$. (The matrix $\tilde{G}$ has thus $2 m+2 n-\nu$ eigenvalues denoted by $\alpha_{i} / \beta_{i}$, all finite). Among these factors, let there be $2 m+2 n-s$ of them (with $0 \leq s \leq \nu$ ) for which $\beta_{i} \neq 0$ and $\left|\alpha_{i} / \beta_{i}\right| \leq 1$, so that there are $s-\nu$ factors for which $\beta_{i} \neq 0$ and $\left|\alpha_{i} / \beta_{i}\right|>1$. The latter $s-\nu$ factors necessarily have $\alpha_{i} \neq 0$ and $\left|\beta_{i} / \alpha_{i}\right|<1$.

Recalling that $\tilde{G}$ is in real Jordan from, this implies that it is possible to partition it as

$$
\left[\begin{array}{cc}
\tilde{G}_{1} & 0 \\
0 & \tilde{G}_{2}
\end{array}\right]
$$

where $\tilde{G}_{1} \in \mathbb{R}^{(s-\nu) \times(s-\nu)}$ is a block-diagonal matrix with eigenvalues satisfying $\left|\alpha_{i} / \beta_{i}\right|>1, \beta_{i} \neq 0$, and $\tilde{G}_{2} \in \mathbb{R}^{(2 m+2 n-s) \times(2 m+2 n-s)}$ is a block-diagonal matrix with eigenvalues satisfying $\left|\alpha_{i} / \beta_{i}\right| \leq 1$, $\beta_{i} \neq 0$. Since all eigenvalues of $\tilde{G}_{1}$ are nonzero, the matrix $\tilde{G}_{1}$ is non-singular. Combining the $s-\nu$ factors associated with $\tilde{G}_{1}$ with the $\nu$ factors associated with the matrix pencil $\lambda I-\rho \tilde{H}$ constitutes $s$ factors for which $\alpha_{i} \neq 0$ and $\left|\beta_{i} / \alpha_{i}\right|<1$.

It follows that the $2(n+m) \times 2(n+m)$ real matrix

$$
\bar{U} \equiv\left[\begin{array}{ccc}
I_{\nu} & 0 & 0 \\
0 & \tilde{G}_{1}^{-1} & 0 \\
0 & 0 & I_{2 m+2 n-s}
\end{array}\right] \tilde{U}
$$


is non-singular and satisfies (A.10), where $\Omega \equiv \tilde{G}_{2}$ is a $(2 m+2 n-s) \times(2 m+2 n-s)$ block-diagonal matrix in real Jordan form with blocks corresponding to the factors of (A.9) for which $\left|\alpha_{i} / \beta_{i}\right| \leq 1$. This implies that

$$
\|\Omega\| \leq 1
$$

The matrix $\Lambda^{\prime} \equiv\left[\begin{array}{cc}\tilde{H} & 0 \\ 0 & \tilde{G}_{1}^{-1}\end{array}\right]$ is a $s \times s$ block-diagonal matrix in real Jordan form with $\nu$ zero eigenvalues (i.e., the eigenvalues of $\tilde{H}$ corresponding to the $\nu$ factors $\left(\alpha_{i} \lambda-\beta_{i} \rho\right)$ for which $\beta_{i}=0$ ), and another $s-\nu$ eigenvalues corresponding to the roots $\beta_{i} \neq 0,\left|\beta_{i} / \alpha_{i}\right|<1$. Thus all $s$ eigenvalues of $\Lambda$ satisfy $\left|\beta_{i} / \alpha_{i}\right|<1$, so that

$$
\|\Lambda\|<1
$$

Because the inequality (A.13) is strict, there also exist values $\delta>1$ such that

$$
\|\delta \Lambda\|<1 .
$$

In what follows, we shall consider a value of $\delta>1$ that is small enough for both (2.14) and (A.14) to hold.

Now let the matrices $\bar{U}, \bar{V}$ be partitioned conformably with the partitions in (A.10):

$$
\left.\bar{U}=\left[\begin{array}{l}
\bar{U}_{1} \\
\bar{U}_{2}
\end{array}\right]\right\} \begin{aligned}
& s \text { rows } \\
& 2 m+2 n-s \text { rows }
\end{aligned}, \quad \bar{V}=\left[\begin{array}{ll}
\bar{V}_{1} & \bar{V}_{2}
\end{array}\right]
$$

where $\bar{V}_{1}$ and $\bar{V}_{2}$ are respectively $2(n+m) \times s$ and $2(n+m) \times(2 m+2 n-s)$ matrices. It follows from the non-singularity of $\bar{U}$ and $\bar{V}$ that the columns of $\left[\begin{array}{cc}\bar{U}_{1}^{\prime} & \bar{U}_{2}^{\prime}\end{array}\right]$ form a basis for $\mathbb{R}^{2(n+m)}$, as do the columns of $\left[\begin{array}{ll}\bar{V}_{1} & \bar{V}_{2}\end{array}\right]$. Hence we can represent $d_{t}$ as

$$
d_{t}=\left[\begin{array}{ll}
\bar{V}_{1} & \bar{V}_{2}
\end{array}\right]\left[\begin{array}{l}
\psi_{t} \\
\phi_{t}
\end{array}\right]
$$

where $\psi_{t}$ is of dimension $s$ and $\phi_{t}$ is of dimension $2 m+2 n-s$. The vectors $\left(\psi_{t}, \phi_{t}\right)$ can be uniquely re-constructed from the vector $d_{t}$, and vice versa.

The decomposition (A.10) defines stable and unstable subspaces for the matrix pencil $\bar{M}-\mu \bar{N}$. In particular, for any $\delta \geq 1$, let us define the $\delta$-stable subspace $\mathcal{D}_{\delta}$ as the set of values $d_{t_{0}}$ for which there exists a deterministic sequence $\left\{d_{t}\right\}$ for $t \geq t_{0}$ consistent with this value of $d_{t_{0}}$, satisfying

$$
\bar{M} d_{t+1}=\bar{N} d_{t}
$$

for all $t \geq t_{0}$, and such that

$$
\lim _{t \rightarrow \infty} \delta^{t} d_{t}=0
$$

(In the case that $\delta=1$, we shall call $\mathcal{D} \equiv \mathcal{D}_{1}$ simply the stable subspace.) We then have the following result regarding the dimension of this linear space.

Lemma 8 Given Assumptions $2(b)$ and 3, let $\mathcal{D}_{\delta}$ be the $\delta$-stable subspace of the matrix pencil $\bar{M}-\mu \bar{N}$ corresponding to a value of $\delta$ such that (A.14) holds. Then $\mathcal{D}_{\delta}$ is a linear space of dimension $s$, the dimension of the square matrix $\Lambda$ in (A.10). 
Proof. Under Assumptions 2(b) and 3, Lemma 8 holds, and we can then rewrite (A.10) as:

$$
\begin{array}{lll}
\bar{U}_{1} \bar{M} \bar{V}_{1}=I_{s}, & \bar{U}_{1} \bar{M} \bar{V}_{2}=0 \\
\bar{U}_{2} \bar{M} \bar{V}_{1}=0, & \bar{U}_{2} \bar{M} \bar{V}_{2}=\Omega
\end{array}
$$

and

$$
\begin{array}{ll}
\bar{U}_{1} \bar{N} \bar{V}_{1}=\Lambda^{\prime}, & \bar{U}_{1} \bar{N} \bar{V}_{2}=0 \\
\bar{U}_{2} \bar{N} \bar{V}_{1}=0, & \bar{U}_{2} \bar{N} \bar{V}_{2}=I_{2 m+2 n-s} .
\end{array}
$$

We observe from these orthogonality relations that the inverse transformations can be written as

$$
\bar{U}^{-1}=\left[\begin{array}{ll}
\bar{M} \bar{V}_{1} & \bar{N} \bar{V}_{2}
\end{array}\right], \quad \bar{V}^{-1}=\left[\begin{array}{c}
\bar{U}_{1} \bar{M} \\
\bar{U}_{2} \bar{N}
\end{array}\right]
$$

(Here we use the fact that because $\bar{U}$ and $\bar{V}$ are non-singular, we know that unique inverses exist.)

We can then pre-multiply the equations in (A.10) by $\bar{U}^{-1}$, using (A.23), to obtain:

$$
\begin{aligned}
\bar{M} \bar{V}_{2} & =\bar{N} \bar{V}_{2} \Omega \\
\bar{M} \bar{V}_{1} \Lambda^{\prime} & =\bar{N} \bar{V}_{1}
\end{aligned}
$$

We can similarly post-multiply the equations in (A.10) by $\bar{V}^{-1}$, using (A.23), to obtain:

$$
\begin{aligned}
\Lambda^{\prime} \bar{U}_{1} \bar{M} & =\bar{U}_{1} \bar{N} \\
\bar{U}_{2} \bar{M} & =\Omega \bar{U}_{2} \bar{N} .
\end{aligned}
$$

Because $\bar{U}^{-1}$ and $\bar{V}^{-1}$ must be non-singular matrices, we observe from (A.23) that $\bar{M} \bar{V}_{1}, \bar{N} \bar{V}_{2}$, $\bar{M}^{\prime} \bar{U}_{1}^{\prime}$, and $\bar{N}^{\prime} \bar{U}_{2}^{\prime}$ must each be matrices of full rank.

Pre-multiplying (A.17) by $\bar{U}_{2}$ and using (A.27), we obtain

$$
\Omega \bar{U}_{2} \bar{N} d_{t+1}=\bar{U}_{2} \bar{N} d_{t}
$$

for each $t \geq t_{0}$. Then using (A.16) to substitute for $d_{t}$ on both sides of this equation, and using (A.22), we obtain

$$
\Omega \phi_{t+1}=\phi_{t}
$$

which in turn implies that

$$
\delta^{t} \phi_{t}=\left(\delta^{-1} \Omega\right) \delta^{t+1} \phi_{t+1}
$$

for each $t \geq t_{0}$. Repeated application of (A.28) implies that

$$
\delta^{t} \phi_{t}=\left(\delta^{-1} \Omega\right)^{k} \delta^{t+k} \phi_{t+k}
$$

for arbitrary $k \geq 1$. Then in the case of any sequence $\left\{d_{t}\right\}$ satisfying (A.18), (A.12) implies that the right-hand side of (A.29) converges to zero for large $k$. Hence we must have $\phi_{t}=0$ for all $t \geq t_{0}$ in the case of any such sequence. Thus $d_{t}$ must be a vector of the form $d_{t}=\bar{V}_{1} \psi_{t}$ for all $t$.

Pre-multiplying (A.17) by $\bar{U}_{1}$ and again using (A.16) to substitute for $d_{t}$, one can similarly show that

$$
\psi_{t+1}=\Lambda^{\prime} \psi_{t}
$$

for all $t \geq t_{0}$. Given a vector $\psi_{t_{0}}$, this law of motion can be solved for the complete sequence $\left\{\psi_{t}\right\}$ and hence for the implied sequence $\left\{d_{t}\right\}$. Since

$$
\delta^{t} \psi_{t}=\left(\delta \Lambda^{\prime}\right)^{t-t_{0}} \delta^{t_{0}} \psi_{t_{0}}
$$


for any $t$, it follows from (A.14) that (A.18) must be satisfied. Hence the $\delta$-stable subspace $\mathcal{D}_{\delta}$ consists of all vectors of the form $d_{t_{0}}=\bar{V}_{1} \psi_{t_{0}}$ for some vector $\psi_{t_{0}}$. Since $\bar{V}$ is invertible, this linear space must be of dimension $s$ (the number of columns of $\bar{V}_{1}$ ).

We turn now to a further characterization of the dimension $s$. Since $\bar{M}-\mu \bar{N}$ is a regular pencil, a pair $(\lambda, \rho)$ determines an eigenvalue $\mu$ of $\bar{M}-\mu \bar{N}$ if $\operatorname{det}[\lambda \bar{M}-\rho \bar{N}]=0$ and $\rho-\lambda \mu=0$. (In particular, a pair $(\lambda, \rho)$ determines an infinite eigenvalue of $\bar{M}-\mu \bar{N}$ if $\operatorname{det}[\lambda \bar{M}-\rho \bar{N}]=0$, and $\rho \neq 0, \lambda=0$.) Because of the symmetries in the elements of the matrices $\bar{M}$ and $\bar{N}$, the eigenvalues of the pencil $\bar{M}-\mu \bar{N}$ also satisfy the following symmetry. ${ }^{28}$

Lemma 9 Given Assumptions 2(b) and 3, the set of values of $\mu=\rho / \lambda$ for which $\operatorname{det}[\lambda \bar{M}-\rho \bar{N}]=0$ is such that if $\mu$ belongs to the set, so do the numbers $\beta \mu^{-1}$, and the complex conjugates $\bar{\mu}$ and $\overline{\beta \mu^{-1}}$. In particular, if the equation holds for $\rho=0$ (and arbitrary $\lambda$ ), then it also holds for $\lambda=0$ (and arbitrary $\rho$ ).

Proof. Given Assumptions 2(b) and 3, Lemma 6 implies that the matrix pencil $\bar{M}-\mu \bar{N}$ is regular. Hence the matrix pencil $\bar{M}-\mu \hat{N}$ where $\hat{N} \equiv \beta^{1 / 2} \bar{N}$ is also regular. Let us define the $2(n+m) \times 2(n+m)$ matrix

$$
J \equiv\left[\begin{array}{cc}
0 & I_{n+m} \\
-I_{n+m} & 0
\end{array}\right]
$$

and observe that

$$
\bar{M}^{\prime} J \bar{M}=\hat{N}^{\prime} J \hat{N}
$$

so that the transposed matrix pencil $(\bar{M}-\mu \hat{N})^{\prime}$ is symplectic. It follows that the generalized eigenvalues of the transposed pencil $(\bar{M}-\mu \hat{N})^{\prime}$ are symmetric with respect to the unit circle (see Theorems 4 and 5 of Pappas, Laub and Sandell, 1980): if $\mu \in \mathbb{C}$ is a generalized eigenvalue of the real matrix pencil $(\bar{M}-\mu \hat{N})^{\prime}$, then so are $\mu^{-1}$ and the complex conjugates $\bar{\mu}, \overline{\mu^{-1}}$. In particular, if $\mu=0$ is an eigenvalue of $(\bar{M}-\mu \hat{N})^{\prime}$, so is $\mu=\infty$.

Since $\operatorname{det}[\bar{M}-\mu \hat{N}]=\operatorname{det}\left[\bar{M}^{\prime}-\mu \hat{N}^{\prime}\right]$ for all $\mu$, it follows that if $\mu \in \mathbb{C}$ is an eigenvalue of $(\bar{M}-\mu \hat{N})$, then so are $\mu^{-1}$ and the complex conjugates $\bar{\mu}, \overline{\mu^{-1}}$. Moreover, $\operatorname{det}[\lambda \bar{M}-\rho \hat{N}]=0$ if and only if $\operatorname{det}\left[\lambda \bar{M}-\beta^{1 / 2} \rho \bar{N}\right]=0$. Hence $\mu$ is a generalized eigenvalue of $(\bar{M}-\mu \bar{N})$ if and only if $\beta^{-1 / 2} \mu$ is a generalized eigenvalue of the transformed pencil $(\bar{M}-\mu \hat{N})$. It then follows that $\beta \mu^{-1}$, $\bar{\mu}$, and $\overline{\beta \mu^{-1}}$ must also be generalized eigenvalues of $(\bar{M}-\mu \bar{N})$.

Lemma 10 Given Assumptions 1(b), 2(b) and 3, the dimension of the square matrix $\Lambda$ in the decomposition (A.10) must be exactly $s=m+n$. Hence the matrix pencil $\bar{M}-\mu \bar{N}$ has exactly $m+n$ generalized eigenvalues satisfying $|\mu|<\beta$ and another $m+n$ generalized eigenvalues (some of which may be infinite) satisfying $|\mu|>1$, and the stable subspace $\mathcal{D}$ is of dimension $m+n$. The dimension of the square matrix $\Omega$ is also $m+n$, and this matrix satisfies

$$
\|\Omega\|<\beta .
$$

Proof. Assumption 1(b) implies that for any initial conditions close enough to consistency with the optimal steady state, there must exist a solution to the first-order conditions (for the deterministic

\footnotetext{
${ }^{28}$ This demonstration that the eigenvalues come in "reciprocal pairs" extends to our environment is a standard result in the theory of linear-quadratic optimal control (e.g., Hansen and Sargent, 2010, chap. 8).
} 
case in which $\xi_{t}=\bar{\xi}$ at all times) in which (2.14) holds. It follows that for arbitrary initial conditions $f_{t_{0}}$, there must be a sequence $\left\{d_{t}\right\}$ satisfying the linearized FOCs (A.17) for all $t \geq t_{0}$, such that $d_{t_{0}}$ is consistent with (A.6), and such that $\left\{\delta^{t} d_{t}\right\}$ is a bounded sequence. ${ }^{29}$ It is then furthermore possible to choose a $\delta>1$ (possibly slightly smaller than the $\delta$ referred to in Assumption 1(b)) such that (A.18) is satisfied. For a small enough choice of $\delta>1$, (A.14) must hold as well. Hence there must exist $\delta>1$ for which (A.14) holds, and such that for any initial conditions $f_{t_{0}}$, there exists a vector $d_{t_{0}}$ in the $\delta$-stable subspace $\mathcal{D}_{\delta}$ consistent with (A.6).

It follows from our characterization of the $\delta$-stable subspace in the proof of Lemma 8 that there must exist a vector $\psi_{t_{0}}$ such that

$$
\left[F_{d} \bar{V}_{1}\right] \psi_{t_{0}}=f_{t_{0}}
$$

It is easily seen that any values for the $m+n$ elements of $f_{t_{0}}$ can be arranged through a suitable specification of the $n-k$ elements of $\tilde{\Theta}_{t_{0}-1}$, the $k$ elements of $\bar{F}_{t_{0}}$, and the $m$ elements of $\tilde{y}_{t_{0}-1}$. Hence the right-hand side of (A.31) can be any element of $\mathbf{R}^{m+n}$. Then in order for a solution to exist for arbitrary initial conditions, it is necessary that

$$
\operatorname{rank} F_{d} \bar{V}_{1}=m+n \text {. }
$$

This requires that $s \geq m+n$.

We further note that the decomposition (A.10) implies that the generalized eigenvalues of the pencil $\bar{M}-\mu \bar{N}$ consist of the $2 m+2 n-s$ eigenvalues $\mu_{i}$ of the matrix $\Omega$ and the reciprocals of the $s$ eigenvalues $\lambda_{j}$ of the matrix $\Lambda$. Lemma 10 implies that for each eigenvalue $\lambda_{j}$ of $\Lambda, \beta \lambda_{j}$ must also be a generalized eigenvalue of the pencil $\bar{M}-\mu \bar{N}$; and since $\left|\lambda_{j}\right|<1$, this must be a generalized eigenvalue with modulus less than $\beta$, and therefore an eigenvalue of $\Omega$ rather than the reciprocal of any eigenvalue of $\Lambda$. Hence for each eigenvalue $\lambda_{j}$ of $\Lambda, \beta \lambda_{j}$ must be an eigenvalue of $\Omega$. This requires that $\Omega$ be of at least the dimension of $\Lambda$, and hence that $s \leq m+n$. Therefore $s=m+n$ exactly. The matrix $\Omega$ is of dimension $m+n$, and its eigenvalues all satisfy $|\mu|<\beta$, which implies (A.30).

Finally, it follows that $\left[F_{d} \bar{V}_{1}\right]$ must be a non-singular square matrix, so that (A.31) can be solved for $\psi_{t_{0}}$ for any specification of the initial conditions $f_{t_{0}}$. Since the largest eigenvalue of $\Lambda$ must have a modulus strictly less than 1 , any initial condition of the form $d_{t_{0}}=\bar{V}_{1} \psi_{t_{0}}$ gives rise to a sequence $\left\{d_{t}\right\}$ satisfying (A.18) for $\delta=1$. Hence this linear space of dimension $m+n$ corresponds to the stable subspace.

In the proof of Lemma 10, it has already been established that for any initial conditions $f_{t_{0}}$, there exists a deterministic solution $\left\{d_{t}\right\}$ to the linearized FOCs that converges exponentially to the steady state for large $t$. This result can then be directly extended to the case of bounded fluctuations in the exogenous disturbances $\left\{\tilde{\xi}_{t}\right\}$, yielding the result stated in the proposition.

Given a bounded stochastic process $\left\{\tilde{\xi}_{t}\right\}$ for the exogenous disturbances and a vector $f_{t_{0}}$ of initial conditions, we are interested in stochastic processes $\left\{d_{t}\right\}$ such that (i) $\left\{d_{t}\right\}$ is bounded; (ii) (A.3) is satisfied for all $t \geq t_{0}$; (iii) (A.5) is satisfied for all $t>t_{0}$; and (iv) $d_{t_{0}}$ satisfies (A.6). Pre-multiplying (A.3) by $\bar{U}_{2}$, we can show as in the proof of Lemma 8 that

$$
\Omega \bar{U}_{2} \bar{N} E_{t} d_{t+1}=\bar{U}_{2} \bar{N} d_{t}-\bar{U}_{2} \bar{N}_{s} \bar{s}_{t}
$$

or equivalently that

$$
E_{t}\left[\left(I-\Omega L^{-1}\right) \bar{U}_{2} \bar{N} d_{t}\right]=\bar{U}_{2} \bar{N}_{s} \bar{s}_{t}
$$

\footnotetext{
${ }^{29}$ Note that convergence in the exact nonlinear dynamics only implies that the sequence must not explode in the linearized dynamics, since the rate of convergence might asymptotically decrease to zero.
} 
Using (A.16) to substitute for $d_{t}$, this can alternatively be written

$$
E_{t}\left[\left(I-\Omega L^{-1}\right) \phi_{t}\right]=\bar{U}_{2} \bar{N}_{s} \bar{s}_{t}
$$

Because of (A.30), the operator $I-\Omega L^{-1}$ is invertible on the linear space of bounded processes $\left\{\phi_{t}\right\}$, so that for any disturbance process such that $\|\bar{s}\|<\infty$, (A.33) has a unique solution such that $\|\phi\|<\infty$, given by

$$
\phi_{t}=E_{t}\left[\left(I-\Omega L^{-1}\right)^{-1} \bar{U}_{2} \bar{N}_{s} \bar{s}_{t}\right]
$$

Similarly, pre-multiplying (A.3) by $\bar{U}_{1}$ and using (A.16) to substitute for $d_{t}$ yields

$$
E_{t} \psi_{t+1}=\Lambda^{\prime} \psi_{t}-\bar{U}_{1} \bar{N}_{s} \bar{s}_{t}
$$

Using (A.16) to substitute for $d_{t}$ in (A.5), and shifting the time index by one period, yields

$$
F_{d} \bar{V}_{1}\left[\psi_{t+1}-E_{t} \psi_{t+1}\right]=F_{s}\left[\bar{s}_{t}-E_{t-1} \bar{s}_{t}\right]-F_{d} \bar{V}_{2}\left[\phi_{t+1}-E_{t} \phi_{t+1}\right]
$$

for each $t \geq t_{0}$. Since $F_{d} \bar{V}_{1}$ is an invertible square matrix (as shown in the proof of Lemma 10), this can be solved uniquely for $\psi_{t+1}$. Substituting expression (A.35) for the conditional expectation $E_{t} \psi_{t+1}$ in this equation, and the solution (A.34) for both $\phi_{t+1}$ and its conditional expectation, we obtain a law of motion of the form

$$
\psi_{t+1}=\Lambda^{\prime} \psi_{t}+r_{t+1}
$$

for all $t \geq t_{0}$, where $\left\{r_{t}\right\}$ is a process satisfying $\|r\|<\infty$ that has been uniquely determined as a function of the evolution of the exogenous disturbances.

Finally, using (A.16) to substitute for $d_{t_{0}}$ in (A.6) we obtain

$$
F_{d} \bar{V}_{1} \psi_{t_{0}}=f_{t_{0}}-F_{d} \bar{V}_{2} \phi_{t_{0}}
$$

Using the solution (A.34) to substitute for $\phi_{t_{0}}$ in this equation, the invertibility of $F_{d} \bar{V}_{1}$ implies that this equation has a unique solution for $\psi_{t_{0}}$ for any specification of the initial conditions $f_{t_{0}}$ and the process for the exogenous disturbances. Given this initial condition for $\psi_{t_{0}}$, the law of motion (A.36) can then be integrated forward, yielding a unique solution for the evolution of $\left\{\psi_{t}\right\}$ for all $t \geq t_{0}$. It follows from (A.14) and the fact that $\|r\|<\infty$ that this solution will satisfy $\|\psi\|<\infty$. Our solutions for the processes $\left\{\phi_{t}, \psi_{t}\right\}$ then imply a unique solution for the process $\left\{d_{t}\right\}$, using (A.16), and the bounds satisfied by the two solutions imply that $\|d\|<\infty$ as well. Hence there is a unique solution satisfying this bound. QED.

\section{A.2 Proof of Lemma 2}

Using the definition of $q_{t}$ and the fact that the matrix $W$ is symmetric, we may rewrite the objective function (4.3) as

$$
\pi\left(y_{t} ; \xi_{t}\right)=-\frac{1}{2}\left[y_{t}^{\prime} w W w^{\prime} y_{t}-2 q^{*}\left(\xi_{t}\right)^{\prime} W w^{\prime} y_{t}+q^{*}\left(\xi_{t}\right)^{\prime} W q^{*}\left(\xi_{t}\right)\right]
$$

so that

$$
\begin{aligned}
D_{1} \pi\left(y_{t} ; \xi_{t}\right) & =-\left[y_{t}^{\prime} w-q^{*}\left(\xi_{t}\right)^{\prime}\right] W w^{\prime} \\
D_{1}\left[\left(D_{1} \pi\left(y_{t} ; \xi_{t}\right)\right)^{\prime}\right] & =-w W w^{\prime} \\
D_{2}\left[\left(D_{1} \pi\left(y_{t} ; \xi_{t}\right)\right)^{\prime}\right] & =w W\left[D q^{*}\left(\xi_{t}\right)\right] .
\end{aligned}
$$


The fact that the targets $q^{*}\left(\xi_{t}\right)$ are achievable implies that in steady state, $D_{1} \pi(\bar{y}, \bar{\xi})=0$, using (4.4) and (A.37). It then follows from (2.13) that

$$
\bar{\varphi}^{\prime}(\bar{I}-\beta \bar{A})=0 .
$$

Assumption 2(b) then implies that $\bar{\varphi}=0$, so that $S, R$ and $B(L)$ reduce to

$$
S=-w W w^{\prime}, \quad R=0, \quad B(L)=w W\left[D q^{*}(\bar{\xi})\right] \cdot L .
$$

The target variables and target values are then given by

$$
\begin{aligned}
\tau_{t} & =w W w^{\prime} \tilde{y}_{t}=w W\left(q_{t}-\bar{q}\right), \\
\tau_{t}^{*} & =w W\left[D q^{*}(\bar{\xi})\right] \cdot \tilde{\xi}_{t},
\end{aligned}
$$

where $\bar{q} \equiv w^{\prime} \bar{y}$. Performing a first-order approximation to $q^{*}\left(\xi_{t}\right)$, we obtain

$$
q_{t}^{*} \equiv q^{*}\left(\xi_{t}\right)=q^{*}(\bar{\xi})+\left[D q^{*}(\bar{\xi})\right] \cdot \tilde{\xi}_{t}+\mathcal{O}\left(\epsilon^{2}\right)
$$

so that, using (4.4), we have

$$
\left[D q^{*}(\bar{\xi})\right] \cdot \tilde{\xi}_{t}=q_{t}^{*}-\bar{q}+\mathcal{O}\left(\epsilon^{2}\right) .
$$

Using this, we can express the "target gaps" $\tau_{t}-\tau_{t}^{*}$ as

$$
\tau_{t}-\tau_{t}^{*}=w W\left(q_{t}-\bar{q}\right)+w W\left(q_{t}^{*}-\bar{q}\right)+\mathcal{O}\left(\epsilon^{2}\right)=w W\left(q_{t}-q_{t}^{*}\right)+\mathcal{O}\left(\epsilon^{2}\right) .
$$

\section{A.3 Proof of Lemma 3}

The fact that the pencil $\bar{A}-\mu \bar{I}$ is of rank $n<m$ (Assumption 2(b)) implies that the columns are linearly dependent, i.e., that there exists $y(\mu)$ such that

$$
[\bar{A}-\mu \bar{I}] y(\mu)=0
$$

for all $\mu$, though by Assumption 2(a), $y(\mu)$ is of order greater than zero. Let $\epsilon_{1} \geq 1$ be the minimal order of solution $y(\mu)$ that exists to (A.38). (A solution of finite order $\epsilon_{1}$ necessarily exists.) Then Theorem 4 of Gantmacher (1959, chap. 12) implies that the pencil $\bar{A}-\mu \bar{I}$ is strictly equivalent to a pencil of the form

$$
\left[\begin{array}{cc}
M_{1}(\mu)^{\prime} & 0 \\
0 & B_{2}^{(1)}-\mu J_{2}^{(1)}
\end{array}\right]
$$

where $B_{2}^{(1)}-\mu J_{2}^{(1)}$ is a pencil for which the equation

$$
\left[B_{2}^{(1)}-\mu J_{2}^{(1)}\right] \hat{y}^{(1)}(\mu)=0
$$

has no solution of order less than $\epsilon_{1}$.

If (A.39) nonetheless has a nonzero solution of minimal order $\epsilon_{2} \geq \epsilon_{1}$, then Theorem 4 of Gantmacher can be applied again to the pencil $B_{2}^{(1)}-\mu J_{2}^{(1)}$. Proceeding in this way, one eventually transforms the pencil $\bar{A}-\mu \bar{I}$ into a pencil of the form shown in (4.6), where the sequence of indices satisfies

$$
\epsilon_{p} \geq \ldots \geq \epsilon_{2} \geq \epsilon_{1} \geq 1
$$

and $\left[B_{2}-\mu J_{2}\right]$ is a pencil for which the equation

$$
\left[B_{2}-\mu J_{2}\right] \hat{y}(\mu)=0
$$


has no nonzero solution, i.e., the columns are linearly independent.

By the same argument as in the proof of Lemma 13, Assumption 2 implies that there is also no vector $\hat{\varphi}(\mu) \neq 0$ such that

$$
\hat{\varphi}(\mu)^{\prime}\left[B_{2}-\mu J_{2}\right]=0,
$$

i.e., the rows of the pencil are also linearly independent. Hence $\left[B_{2}-\mu J_{2}\right]$ must be a square pencil of some dimension $q \times q$. (Note that it is possible that $q=0$, i.e., that $B_{2}$ and $J_{2}$ are null matrices.)

Adding up the columns of the matrix in (4.6), one observes that

$$
\sum_{i=1}^{p} \epsilon_{i}+q=n .
$$

Adding up the rows, one similarly observes that

$$
\sum_{i=1}^{p}\left(\epsilon_{i}+1\right)+q=m,
$$

from which it follows that the number of $M_{i}$ blocks must equal $p=m-n$.

This theorem implies that there exist nonsingular square matrices $P, Q$ of dimensions $n \times n$ and $m \times m$ respectively that satisfy (4.6).

\section{A.4 Proof of Lemma 4}

Consider the case of unidimensional policy so that $i=p=1$, and thus $\epsilon_{i}=\tilde{n}$.

Suppose that (4.8) hold for all $t \geq t_{0}$. The first element of the vector of FOCs (4.8) can be written

$$
\beta^{-1} \hat{\varphi}_{1, t}^{(1)}=\hat{\tau}_{1, t}^{(1)}
$$

while the $j$-th element (for $2 \leq j \leq \tilde{n}$ ) can be written as

$$
\beta^{-1} \hat{\varphi}_{1, t}^{(j)}=\hat{\tau}_{1, t}^{(j)}-E_{t} \hat{\varphi}_{1, t+1}^{(j-1)} .
$$

This system of equations can be solved recursively for the $\left\{\hat{\varphi}_{1, t}^{(j)}\right\}$, yielding

$$
\hat{\varphi}_{1, t}^{(j)}=\beta E_{t}\left[\Gamma^{j}\left(\beta L^{-1}\right) \hat{\tau}_{1, t}\right]
$$

for each $1 \leq j \leq \tilde{n}$, where $\Gamma^{j}(\mu)$ is the $j$-th row of the matrix polynomial $\Gamma(\mu)$. This gives the vector of conditions (4.13).

The $(\tilde{n}+1)$-st element of the vector of FOCs $(4.8)$ states that

$$
E_{t} \hat{\varphi}_{1, t+1}^{(\tilde{n})}=\hat{\tau}_{1, t}^{(\tilde{n}+1)}
$$

Substituting solution (A.42) for $\hat{\varphi}_{1, t}^{(j)}$, we obtain

$$
\hat{\tau}_{1, t}^{(\tilde{n}+1)}=E_{t}\left[\beta L^{-1} \Gamma^{\tilde{n}}\left(\beta L^{-1}\right) \hat{\tau}_{1, t}\right]
$$

which implies (4.14). Here we use the fact that

$$
\delta(\mu)^{\prime}=(-\mu)^{\tilde{n}}\left[e_{\tilde{n}+1}^{\prime}-\mu^{-1} \Gamma^{\tilde{n}}\left(\mu^{-1}\right)\right]
$$


where $e_{\tilde{n}+1}^{\prime}$ is a $1 \times(\tilde{n}+1)$ vector of the form $e_{\tilde{n}+1}^{\prime} \equiv\left[\begin{array}{ll}0 \ldots 0 & 1\end{array}\right]$. Thus both (4.13) and (4.14) must hold in all periods $t \geq t_{0}$.

To prove the converse, suppose that the processes $\left\{\hat{\tau}_{1, t}\right\}$ and $\left\{\hat{\varphi}_{1, t}\right\}$ satisfy (4.13) and (4.14) in all periods $t \geq t_{0}$. Condition (4.13) implies (A.42) for each $1 \leq j \leq \tilde{n}$, which in turn implies condition (A.40), and condition (A.41) for each $2 \leq j \leq \tilde{n}$. These are just the first $\tilde{n}$ elements of the vector of FOCs (4.8). Condition (4.14) implies (A.44), which together with the case $j=\tilde{n}$ of (A.42) implies (A.43). This is just the $(\tilde{n}+1)$-st element of the vector of FOCs (4.8). Thus the entire vector of conditions (4.8) must hold in each period $t \geq t_{0}$.

\section{A.5 Proof of Proposition 2}

To prove Proposition 2, it will be useful to appeal to the following Lemma.

Lemma 11 Suppose that the processes $\left\{\hat{\tau}_{1, t}\right\}$ and $\left\{\hat{\varphi}_{1, t}\right\}$ satisfy (4.13) for all $t \geq t_{0}$. Then for any $1 \leq j \leq \tilde{n}$, and any $t \geq t_{0}$,

$$
E_{t} z_{t+j-1}-E_{t-1} z_{t+j-1}=-(-\beta)^{-j}\left[\hat{\varphi}_{1, t}^{(j)}-E_{t-1} \hat{\varphi}_{1, t}^{(j)}\right]
$$

where $\hat{\varphi}_{1, t}^{(j)}$ is the $j$-th element of the vector $\hat{\varphi}_{1, t}$. When $t=t_{0}$, the expression $E_{t_{0}-1} \hat{\varphi}_{1, t_{0}}^{(j)}$ is taken to refer to the historical expectations $\beta E_{t_{0}-1}\left[\Gamma^{j}\left(\beta L^{-1}\right) \hat{\tau}_{1, t_{0}}\right]$. It follows that

$$
w_{t}=-\left(\hat{\varphi}_{1, t}-E_{t-1} \hat{\varphi}_{1, t}\right) \text {. }
$$

for any $t \geq t_{0}$, where $w_{t}$ is defined in (4.15).

Proof. Using the definition (4.12) we have

$$
\begin{aligned}
E_{t} z_{t+j-1}-E_{t-1} z_{t+j-1}= & E_{t}\left[\hat{\tau}_{1, t+j-1}^{(1)}-\beta^{-1} \hat{\tau}_{1, t+j-2}^{(2)}+\ldots+\left(-\beta^{-1}\right)^{j-1} \hat{\tau}_{1, t}^{(j)}\right] \\
& -E_{t-1}\left[\hat{\tau}_{1, t+j-1}^{(1)}-\beta^{-1} \hat{\tau}_{1, t+j-2}^{(2)}+\ldots+\left(-\beta^{-1}\right)^{j-1} \hat{\tau}_{1, t}^{(j)}\right] \\
= & \left(-\beta^{-1}\right)^{j-1}\left\{E_{t}\left[\Gamma^{j}\left(\beta L^{-1}\right) \hat{\tau}_{1, t}\right]-E_{t-1}\left[\Gamma^{j}\left(\beta L^{-1}\right) \hat{\tau}_{1, t}\right]\right\} \\
= & -(-\beta)^{-j}\left[\hat{\varphi}_{1, t}^{(j)}-E_{t-1} \hat{\varphi}_{1, t}^{(j)}\right] .
\end{aligned}
$$

Here, the first equality uses the definition of $\delta(\mu)^{\prime}$, and the fact that $E_{t} \hat{\tau}_{1, t-k}=E_{t-1} \hat{\tau}_{1, t-k}$ for any $k \geq 1$. The second equality uses the definition of $\Gamma(\mu)$, denotes by $\Gamma^{j}(\mu)$ its $j$-th row. The third equality uses (4.13). In the case that $t=t_{0}$, the replacement of $\beta E_{t-1}\left[\Gamma^{j}\left(\beta L^{-1}\right) \hat{\tau}_{1, t}\right]$ by $E_{t-1} \hat{\varphi}_{1, t}^{(j)}$ is justified under the definition of $E_{t_{0}-1} \hat{\varphi}_{1, t_{0}}^{(j)}$ proposed above. Equation (A.46) is obtained by replacing $(-\beta)^{j}\left(E_{t} z_{t+j-1}-E_{t-1} z_{t+j-1}\right)$ with $-\left(\hat{\varphi}_{1, t}^{(j)}-E_{t-1} \hat{\varphi}_{1, t}^{(j)}\right)$ in the definition of $w_{t}$ in equation (4.15).

We may now proceed with the proof of Proposition 2. Condition (4.8) implies that (4.13) and (4.14) must hold for all $t \geq t_{0}$, using Lemma 4. The fact that (4.13) holds implies that (A.45) must also hold for all $t \geq t_{0}$, using Lemma 11 .

Let us first consider any period $t \geq t_{0}+\tilde{n}-k_{1}$. Then

$$
\begin{aligned}
E_{t} z_{t+k_{1}} & =\sum_{j=1}^{\tilde{n}-k_{1}}\left(E_{t+1-j} z_{t+k_{1}}-E_{t-j} z_{t+k_{1}}\right)+E_{t-\left(\tilde{n}-k_{1}\right)} z_{t+k_{1}} \\
& =\sum_{j=1}^{\tilde{n}-k_{1}}(-\beta)^{-\left(k_{1}+j\right)} w_{t+1-j}^{\left(k_{1}+j\right)} .
\end{aligned}
$$


Here the second line uses the definition of $w_{t}$ to replace the first term on the right-hand side of the first line, denoting by $w_{t}^{(j)}$ the $j$-th element of $w_{t}$, and uses (4.14) to eliminate the second term. It follows from (A.46) that (4.10) can be rewritten as $V_{2} w_{t}=0$.

Premultiplying on both sides by $-V_{22}^{-1}$ yields

$$
\left[\begin{array}{ll}
\Phi & -I
\end{array}\right] w_{t}=0
$$

where $\Phi \equiv-V_{22} V_{21}$ is a $\left(\tilde{n}-k_{1}\right) \times k_{1}$ matrix, so that the vector $w_{t}$ can be reconstructed from its first $k_{1}$ elements, $\bar{w}_{t}$ :

$$
w_{t}=\left[\begin{array}{c}
I_{k_{1}} \\
\Phi
\end{array}\right] \bar{w}_{t}
$$

Using this last expression, we can rewrite (A.47) as

$$
\begin{aligned}
E_{t} z_{t+k_{1}} & =\sum_{j=1}^{\tilde{n}-k_{1}}(-\beta)^{-\left(k_{1}+j\right)} \phi_{j}^{\prime} \bar{w}_{t+1-j} \\
& =(-\beta)^{-k_{1}} \sum_{j=1}^{\tilde{n}-k_{1}} \phi_{j}^{\prime} \Omega_{t}^{j} \\
& =(-\beta)^{-k_{1}} \operatorname{tr}\left[\Phi \Omega_{t}\right],
\end{aligned}
$$

which establishes (4.17). Here we use the notation $\phi_{j}^{\prime}$ for the $j$-th row of $\Phi$ and the notation $\Omega_{t}^{j}$ for the $j$-th column of $\Omega_{t}$.

Let us now consider any period $t_{0} \leq t<t_{0}+\tilde{n}-k_{1}$. Then

$$
\begin{aligned}
E_{t} z_{t+k_{1}} & =\sum_{j=1}^{t-t_{0}}\left(E_{t+1-j} z_{t+k_{1}}-E_{t-j} z_{t+k_{1}}\right)+E_{t_{0}} z_{t+k_{1}} \\
& =(-\beta)^{-k_{1}} \sum_{j=1}^{t-t_{0}} \phi_{j}^{\prime} \Omega_{t}^{j}+E_{t_{0}} z_{t+k_{1}},
\end{aligned}
$$

where the second line is obtained using the same reasoning as was used to derive (A.47) and (A.48).

For the given historical expectations $e_{t_{0}-1}$, let $\Xi_{1, t_{0}-1}$ be given by (4.18) where $\chi_{t_{0}-1}$ is the vector whose $j$-th element is given by (4.19). With this definition, (4.11) together with (4.8) and Lemma 4 imply that

$$
V_{2}\left[\hat{\varphi}_{1, t_{0}}\right]=V_{2} E_{t_{0}-1}\left[\hat{\varphi}_{1, t_{0}}\right]+V_{22} \chi_{t_{0}-1} .
$$

Premultiplying by $V_{22}^{-1}$ and recalling that $\Phi \equiv-V_{22}^{-1} V_{21}$ and $w_{t_{0}}=-\left(\hat{\varphi}_{1, t_{0}}-E_{t_{0}-1} \hat{\varphi}_{1, t_{0}}\right)$, using Lemma 11, this yields

$$
\left[\begin{array}{ll}
\Phi & \left(-I_{\tilde{n}-k_{1}}\right)
\end{array}\right] w_{t_{0}}=\chi_{t_{0}-1} .
$$

For any $1 \leq j \leq \tilde{n}-k_{1}$, the $j$-th row of (A.50) can be written

$$
\begin{aligned}
& \phi_{j}^{\prime} \bar{w}_{t_{0}}-(-\beta)^{k_{1}+j}\left[E_{t_{0}} z_{t_{0}+k_{1}+j-1}-E_{t_{0}-1} z_{t_{0}+k_{1}+j-1}\right] \\
= & (-\beta)^{k_{1}+j} E_{t_{0}-1} z_{t_{0}+k_{1}+j-1}-\sum_{i=1}^{\tilde{n}-k_{1}-j}(-\beta)^{-i} \phi_{j+i}^{\prime} \bar{w}_{t_{0}-i},
\end{aligned}
$$

or alternatively,

$$
(-\beta)^{k_{1}+j} E_{t_{0}} z_{t_{0}+k_{1}+j-1}=\sum_{i=0}^{\tilde{n}-k_{1}-j}(-\beta)^{-i} \phi_{j+i}^{\prime} \bar{w}_{t_{0}-i}=\sum_{h=j}^{\tilde{n}-k_{1}}(-\beta)^{j} \phi_{h}^{\prime} \Omega_{t_{0}+j-1}^{h} .
$$


Thus if we let $j=t+1-t_{0}$, we find that

$$
E_{t_{0}} z_{t+k_{1}}=(-\beta)^{-k_{1}} \sum_{h=t-t_{0}+1}^{\tilde{n}-k_{1}} \phi_{h}^{\prime} \Omega_{t}^{h} .
$$

Using this to substitute for the final term on the right-hand side of (A.49), we obtain

$$
E_{t} z_{t+k_{1}}=(-\beta)^{-k_{1}} \sum_{j=1}^{\tilde{n}-k_{1}} \phi_{j}^{\prime} \Omega_{t}^{j},
$$

so that (4.17) is satisfied for each $t_{0} \leq t<t_{0}+\tilde{n}-k_{1}$. Since we have already shown that (4.17) holds for any $t \geq t_{0}+\tilde{n}-k_{1}$, it follows that (4.17) is satisfied for each $t \geq t_{0}$.

\section{A.6 Proof of Lemma 5}

For period $t_{0}$, conditions (4.20) and (4.21) hold by assumption, given the initial condition (4.11) and the initial Lagrange multipliers (4.18). Next, (4.8) implies $\hat{\varphi}_{1, t}=\beta E_{t}\left[\Gamma\left(\beta L^{-1}\right) \hat{\tau}_{1, t}\right]$ at all dates $t \geq t_{0}$, by Lemma 4 . Using this, conditions (4.10) in turn imply that

$$
V_{2} \hat{\varphi}_{1, t}=V_{2} E_{t-1} \hat{\varphi}_{1, t}=V_{2} \beta E_{t-1}\left[\Gamma\left(\beta L^{-1}\right) \hat{\tau}_{1, t}\right]
$$

for all $t>t_{0}$. Equation (4.20) thus holds in all period $t \geq t_{0}$, where $\Xi_{1, t-1}$ is given by (4.21) in period $t=t_{0}$, and

$$
\Xi_{1, t-1}=V_{2} \beta E_{t-1}\left[\Gamma\left(\beta L^{-1}\right) \hat{\tau}_{1, t}\right]
$$

for all $t>t_{0}$. To prove that (4.21) holds at all dates, we need to show that $V_{22} \chi_{t-1}=0$ for all periods $t>t_{0}$.

Given the initial Lagrange multipliers $\Xi_{1, t_{0}}$ defined in (4.18) and using Proposition 2 implies that (4.17) must hold at all dates $t \geq t_{0}$. Condition (4.17) then implies that for any $t \geq t_{0}$ and any $1 \leq j \leq \tilde{n}-k_{1}$,

$$
\begin{aligned}
E_{t} z_{t+k_{1}+j-1} & =E_{t}\left[E_{t+j-1} z_{t+k_{1}+j-1}\right]=(-\beta)^{-k_{1}} \operatorname{tr}\left[\Phi E_{t} \Omega_{t+j-1}\right] \\
& =(-\beta)^{-k_{1}} E_{t}\left[(-\beta)^{-1} \phi_{1}^{\prime} \bar{w}_{t+j-1}+\ldots+(-\beta)^{-\left(\tilde{n}-k_{1}\right)} \phi_{\tilde{n}-k_{1}}^{\prime} \bar{w}_{t+j+k_{1}-\tilde{n}}\right] \\
& =(-\beta)^{-k_{1}}\left[(-\beta)^{-j} \phi_{j}^{\prime} \bar{w}_{t}+\ldots+(-\beta)^{-\left(\tilde{n}-k_{1}\right)} \phi_{\tilde{n}-k_{1}}^{\prime} \bar{w}_{t+j+k_{1}-\tilde{n}}\right] \\
& =(-\beta)^{-\left(k_{1}+j\right)} \sum_{i=0}^{\tilde{n}-k_{1}-j}(-\beta)^{-i} \phi_{j+i}^{\prime} \bar{w}_{t-i} .
\end{aligned}
$$

Using this to substitute for $E_{t-1} z_{t+k_{1}+j-1}$ in (4.19), we obtain

$$
\chi_{t-1}^{j}=\phi_{j}^{\prime} E_{t-1} \bar{w}_{t}=0
$$

for all $t>t_{0}$, and any $1 \leq j \leq \tilde{n}-k_{1}$. It follows that $\chi_{t-1}=0$, and hence that $V_{22} \chi_{t-1}=0$ for all $t>t_{0}$. 


\section{A.7 Proof of Proposition 3}

Proposition 2 implies that fulfillment of the target criterion is a necessary condition for fulfillment of the FOCs, and hence a necessary condition for optimality, in the case of a problem involving any initial conditions $e_{t_{0}-1}$ and initial Lagrange multipliers $\Xi_{1, t_{0}-1}$ defined by (4.18). But consistency of the paths of the target variables with the target criterion (4.17) is also sufficient for satisfaction of the FOCs, as established by the following lemma.

Lemma 12 Suppose that Assumption 4 holds and that the evolution of the target variables $\left\{\hat{\tau}_{1, t}\right\}$ for $t \geq t_{0}$ satisfies the target criterion (4.17) for all $t \geq t_{0}$, given the initial conditions $e_{t_{0}-1}$. Then there exists a Lagrange multiplier process $\left\{\hat{\varphi}_{1, t}\right\}$ for $t \geq t_{0}$ that satisfies conditions (4.8) for all $t \geq t_{0}$, (4.10) for all $t>t_{0}$, and the initial condition (4.11), where the vector $\Xi_{1, t_{0}-1}$ is defined by (4.18).

Proof. Let the process $\left\{\hat{\varphi}_{1, t}\right\}$ be given by (4.13) for all $t \geq t_{0}$. Note that if $k_{1}<\tilde{n}$, satisfaction of (4.17) for all $t \geq t_{0}$ implies that

$$
E_{t} z_{t+\tilde{n}}=E_{t}\left[E_{t+\tilde{n}-k_{1}} z_{t+\tilde{n}}\right]=(-\beta)^{-k_{1}} \operatorname{tr}\left[\Phi E_{t} \Omega_{t+\tilde{n}-k_{1}}\right]=0
$$

for any $t \geq t_{0}$, using the fact that $E_{t} \bar{w}_{t+j}=0$ for any $t \geq t_{0}$ and any $j \geq 1$. But if $k_{1}=\tilde{n}, \Omega_{t}$ is a null matrix, and (4.17) also implies that $E_{t} z_{t+\tilde{n}}=0$ in this case as well. Hence the target criterion (4.17) implies condition (4.14). Then by Lemma 4, the fact that (4.13) and (4.14) hold for all $t \geq t_{0}$ implies that (4.8) holds for all $t \geq t_{0}$.

Recall from (A.51) that condition (4.17) also implies

$$
E_{t} z_{t+k_{1}+j-1}=(-\beta)^{-\left(k_{1}+j\right)} \sum_{i=0}^{\tilde{n}-k_{1}-j}(-\beta)^{-i} \phi_{j+i}^{\prime} \bar{w}_{t-i}
$$

for any $t \geq t_{0}$ and any $1 \leq j \leq \tilde{n}-k_{1}$. It follows that for all $t>t_{0}$ and all $1 \leq j \leq \tilde{n}-k_{1}$ :

$$
E_{t} z_{t+k_{1}+j-1}-E_{t-1} z_{t+k_{1}+j-1}=(-\beta)^{-\left(k_{1}+j\right)} \phi_{j}^{\prime} \bar{w}_{t}
$$

so that

$$
w_{t} \equiv\left[\begin{array}{c}
(-\beta)\left[z_{t}-E_{t-1} z_{t}\right] \\
\vdots \\
(-\beta)^{k_{1}}\left[E_{t} z_{t+k_{1}-1}-E_{t-1} z_{t+k_{1}-1}\right] \\
(-\beta)^{k_{1}+1}\left[E_{t} z_{t+k_{1}}-E_{t-1} z_{t+k_{1}}\right] \\
\vdots \\
(-\beta)^{\tilde{n}}\left[E_{t} z_{t+\tilde{n}-1}-E_{t-1} z_{t+\tilde{n}-1}\right]
\end{array}\right]=\left[\begin{array}{c}
I_{k_{1}} \\
\Phi
\end{array}\right] \bar{w}_{t} .
$$

Premultiplying by $V_{2}$ implies in turn that

$$
V_{2} w_{t}=\left[\begin{array}{ll}
V_{21} & V_{22}
\end{array}\right]\left[\begin{array}{c}
I_{k_{1}} \\
\Phi
\end{array}\right] \bar{w}_{t}=0
$$

for any $t>t_{0}$. For the process $\left\{\hat{\varphi}_{1, t}\right\}$ given by (4.13) for all $t \geq t_{0}$, we can apply Lemma 11 to express $w_{t}$ as

$$
w_{t}=-\left[\hat{\varphi}_{1, t}-E_{t-1} \hat{\varphi}_{1, t}\right]
$$

for all $t \geq t_{0}$. Since $V_{2} w_{t}=0$ for any $t>t_{0}$, equation (4.10) must hold for all $t>t_{0}$. 
To show that the initial condition (4.11) also holds, where the vector $\Xi_{1, t_{0}-1}$ is defined by (4.18), note that (A.51) implies

$$
(-\beta)^{k_{1}+j} E_{t_{0}} z_{t_{0}+k_{1}+j-1}=\sum_{i=0}^{\tilde{n}-k_{1}-j}(-\beta)^{-i} \phi_{j+i}^{\prime} \bar{w}_{t_{0}-i}
$$

for any $1 \leq j \leq \tilde{n}-k_{1}$. Subtracting $\phi_{j}^{\prime} \bar{w}_{t_{0}}+(-\beta)^{k_{1}+j} E_{t_{0}-1} z_{t_{0}+k_{1}+j-1}$ on both sides (where again expectations taken at date $t_{0}-1$ denote historical forecasts) yields

$$
-\phi_{j}^{\prime} \bar{w}_{t_{0}}+(-\beta)^{k_{1}+j}\left[E_{t_{0}} z_{t_{0}+k_{1}+j-1}-E_{t_{0}-1} z_{t_{0}+k_{1}+j-1}\right]=-\chi_{t_{0}-1}^{j}
$$

where $\chi_{t_{0}-1}^{j}$ is the $j$-th element of the vector $\chi_{t_{0}-1}$, defined in (4.19). Since the previous expression holds for any $1 \leq j \leq \tilde{n}-k_{1}$, we may rewrite it in matrix form as

$$
\left[-\Phi \quad I_{\tilde{n}-k_{1}}\right] w_{t_{0}}=-\chi_{t_{0}-1}
$$

using definition (4.15). Using again definition (4.15) and Lemma 11 we can equivalently rewrite this as

$$
\left[\begin{array}{ll}
-\Phi & I_{\tilde{n}-k_{1}}
\end{array}\right]\left[\hat{\varphi}_{1, t_{0}}-E_{t_{0}-1} \hat{\varphi}_{1, t_{0}}\right]=\chi_{t_{0}-1}
$$

or as

$$
V_{2} \hat{\varphi}_{1, t_{0}}=V_{2} E_{t_{0}-1}\left[\hat{\varphi}_{1, t_{0}}\right]+V_{22} \chi_{t_{0}-1},
$$

after premultiplying on both sides by $V_{22}$. Using (4.13) to replace $\hat{\varphi}_{1, t_{0}}$ with $\beta E_{t_{0}}\left[\Gamma\left(\beta L^{-1}\right) \hat{\tau}_{1, t_{0}}\right]$ on the right-hand side yields the initial condition (4.11), where the vector $\Xi_{1, t_{0}-1}$ is defined by (4.18).

The Lagrange multiplier process referred to in the lemma is given by (4.13). Since (4.17) implies (4.14), it follows directly from Lemma 4 that these multipliers satisfy (4.8) for all $t \geq t_{0}$. It then remains only to show that (4.10) and (4.11) are satisfied as well, by reversing the steps used to derive (4.17) from these conditions in the proof of Proposition 2.

Combining Lemma 12 with Proposition 5, one sees that in the case of any bounded process $\left\{\hat{\tau}_{t}\right\}$ consistent with the target criterion (4.17) for all $t \geq t_{0}$, there necessarily exists a bounded multiplier process $\left\{\hat{\varphi}_{t}\right\}$ for $t \geq t_{0}$ that satisfies conditions (4.1) for all $t \geq t_{0}$ and (4.2) for all $t>t_{0}$, and that is consistent with given initial multipliers $\tilde{\Theta}_{t_{0}-1}$, as long as the vector $\tilde{\Theta}_{t_{0}-1}$ is consistent with (4.18). Moreover, since by Proposition 1(a), there is a unique bounded solution to these equations consistent with the given initial multipliers, there must be a unique bounded process $\left\{\hat{\tau}_{t}\right\}$ consistent with the target criterion (4.17) for all $t \geq t_{0}$. (If there were multiple paths $\left\{\hat{\tau}_{t}\right\}$ consistent with the target criterion, each of these would have to correspond to a different solution to the FOCs consistent with the same initial multipliers (4.18). ${ }^{30}$ But this would contradict Proposition 1(a).)

Hence the target criterion (4.17) determines a unique bounded solution for the equilibrium evolution of the endogenous variables. By Proposition 1(b), the evolution determined by this criterion represents a local linear approximation to the optimal evolution, under the modified problem defined by initial Lagrange multipliers (4.18). Finally, this way of choosing the initial multipliers as a function of the initial conditions $e_{t_{0}-1}$ is self-consistent, by Lemma 5 .

\footnotetext{
${ }^{30}$ Note that the initial multipliers $\Xi_{2, t_{0}-1}$ can be chosen arbitrarily, and so could be chosen to be the same in the case of each of these solutions.
} 


\section{A.8 Essential vs. Inessential Variables}

We showed in Section 4.1 that the matrix pencil $\bar{A}-\mu \bar{I}$ admits a canonical decomposition of the form (4.6), where $B_{2}-\mu J_{2}$ is a regular matrix pencil of dimension $q \times q$. We now consider the case in which $q>0$. In the somewhat special case that the equilibrium relations (3.2) are decomposable into two sub-systems of a particular type, some of the endogenous variables are determined independently of any policy choices. We call such variables "inessential." To show this, we make the following technical assumptions.

Assumption 5 Suppose that the decomposition (4.6) of the matrix pencil $\bar{A}-\mu \bar{I}$ satisfies the additional properties:

(a) There exist non-singular matrices $T_{1}(k \times k)$ and $T_{2}((n-k) \times(n-k))$ such that

$$
\left[\begin{array}{cc}
T_{1} & 0 \\
0 & T_{2}
\end{array}\right] P^{\prime}=\left[\begin{array}{cc}
U_{1}^{\prime} & 0 \\
0 & X_{1}^{\prime} \\
U_{2}^{\prime} & 0 \\
0 & X_{2}^{\prime}
\end{array}\right]
$$

where

$$
U^{\prime} \equiv\left[\begin{array}{c}
U_{1}^{\prime} \\
U_{2}^{\prime}
\end{array}\right], \quad X^{\prime} \equiv\left[\begin{array}{l}
X_{1}^{\prime} \\
X_{2}^{\prime}
\end{array}\right]
$$

are non-singular square matrices $((n-q) \times(n-q)$ and $q \times q$, respectively), and the blocks

$$
\left[\begin{array}{cc}
U_{1}^{\prime} & 0 \\
0 & X_{1}^{\prime}
\end{array}\right], \quad\left[\begin{array}{cc}
U_{2}^{\prime} & 0 \\
0 & X_{2}^{\prime}
\end{array}\right]
$$

are $k \times n$, and $(n-k) \times n$ respectively.

(b) Moreover, the characteristic polynomial

$$
P(\lambda, \mu) \equiv \operatorname{det}\left[\lambda J_{2}-\mu B_{2}\right]
$$

can be factored as

$$
P(\lambda, \mu)=c \prod_{i=1}^{k_{2}}\left(\lambda-\gamma_{i} \mu\right) \prod_{j=1}^{q-k_{2}}\left(\mu-\eta_{j} \lambda\right),
$$

where $0 \leq k_{2} \leq q$ is the number of rows of $X_{1}^{\prime}$ in (A.52), $c \neq 0$, and the complex numbers $\left\{\gamma_{i}, \eta_{j}\right\}$ satisfy $\left|\gamma_{i}\right|<1$ for all $i,\left|\eta_{j}\right|<\beta$ for all $j$.

Here the blocks $U_{1}^{\prime}, U_{2}^{\prime}, X_{1}^{\prime}, X_{2}^{\prime}$ are of dimensions

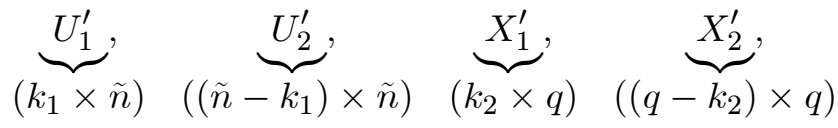

where $\tilde{n} \equiv n-q>0,0 \leq k_{1} \leq k, 0 \leq k_{2} \leq q$, and $k_{1}+k_{2}=k$. For future reference, let us also define

$$
\left[\begin{array}{l}
V_{1} \\
V_{2}
\end{array}\right] \equiv\left[\begin{array}{ll}
U_{1} & U_{2}
\end{array}\right]^{-1}, \quad\left[\begin{array}{l}
W_{1} \\
W_{2}
\end{array}\right] \equiv\left[\begin{array}{ll}
X_{1} & X_{2}
\end{array}\right]^{-1} .
$$

where the blocks $V_{1}, V_{2}, W_{1}$, and $W_{2}$ are of dimensions

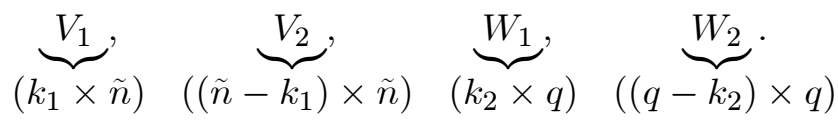


(Note that in the case that $q=0, V_{2}$ reduces to $V_{2}=\left[0,\left(T_{2}^{\prime}\right)^{-1}\right] P^{-1}$, as stated in the main text.)

This additional notation will allow us to decompose the system of equations (4.2) in a way conformable with our decomposition of (3.2) and (4.1).

Under Assumption 2, Lemma 3 states that a decomposition of the form (4.6) exists, so that the system of linearized structural equations (3.2) can equivalently be written in the form

$$
\begin{aligned}
& J_{1} E_{t} y_{1, t+1}^{*}=B_{1} y_{1, t}^{*}+\Gamma_{1} \tilde{\xi}_{t} \\
& J_{2} E_{t} y_{2, t+1}^{*}=B_{2} y_{2, t}^{*}+\Gamma_{2} \tilde{\xi}_{t}
\end{aligned}
$$

for all $t \geq t_{0}$, where

$$
\left[\begin{array}{l}
y_{1, t}^{*} \\
y_{2, t}^{*}
\end{array}\right] \equiv Q^{\prime} \tilde{y}_{t}, \quad\left[\begin{array}{l}
\Gamma_{1} \\
\Gamma_{2}
\end{array}\right] \equiv\left(P^{-1}\right)^{\prime} \bar{C}
$$

Note that $y_{1, t}^{*}, y_{2, t}^{*}$ are two independent vectors of endogenous variables (of lengths $m-q$ and $q$, respectively), that we may refer to as the "essential" and "inessential" endogenous variables respectively. ${ }^{31}$ Each of the subsystems (A.55) and (A.56) involves only one of these sets of endogenous variables. Thus the system (3.2) decomposes into two independent systems of equations for the two distinct sets of endogenous variables. Assumption 5(a) implies that it is also possible to decompose (3.3)-(3.4) into separate systems of equations, using the same decomposition of the state variables $\tilde{y}_{t}$ into $\left(y_{1, t}^{*}, y_{2, t}^{*}\right)$.

The content of (3.3) is unchanged if we pre-multiply both sides by $T_{1}$ (since $T_{1}$ is non-singular). But (4.6), (A.52) imply that

$$
T_{1} \bar{I}_{1}=\left[\begin{array}{cc}
U_{1}^{\prime} & 0 \\
0 & X_{1}^{\prime}
\end{array}\right]\left[\begin{array}{cc}
J_{1} & 0 \\
0 & J_{2}
\end{array}\right] Q^{\prime}=\left[\begin{array}{cc}
U_{1}^{\prime} J_{1} & 0 \\
0 & X_{1}^{\prime} J_{2}
\end{array}\right] Q^{\prime}
$$

so that the system (3.3) can be equivalently written as

$$
\begin{aligned}
U_{1}^{\prime} J_{1}\left(y_{1, t}^{*}-E_{t-1} y_{1, t}^{*}\right) & =\Delta_{1}\left(\tilde{\xi}_{t}-E_{t-1} \tilde{\xi}_{t}\right) \\
X_{1}^{\prime} J_{2}\left(y_{2, t}^{*}-E_{t-1} y_{2, t}^{*}\right) & =\Delta_{2}\left(\tilde{\xi}_{t}-E_{t-1} \tilde{\xi}_{t}\right)
\end{aligned}
$$

for all $t>t_{0}$, where

$$
\left[\begin{array}{c}
\Delta_{1} \\
\Delta_{2}
\end{array}\right] \equiv T_{1} \bar{D}
$$

Similarly, the system (3.4) can equivalently be written as

$$
\begin{aligned}
& U_{1}^{\prime} J_{1} y_{1, t_{0}}^{*}=U_{1}^{\prime} B_{1} y_{1, t_{0}-1}^{*}+\Delta_{1} \tilde{\xi}_{t_{0}}-\beta \Phi_{1, t_{0}} \\
& X_{1}^{\prime} J_{2} y_{2, t_{0}}^{*}=X_{1}^{\prime} B_{2} y_{2, t_{0}-1}^{*}+\Delta_{2} \tilde{\xi}_{t_{0}}-\beta \Phi_{2, t_{0}}
\end{aligned}
$$

for period $t_{0}$, where

$$
\left[\begin{array}{l}
\Phi_{1, t_{0}} \\
\Phi_{2, t_{0}}
\end{array}\right] \equiv T_{1} \bar{F}_{t_{0}}
$$

Thus the system of structural equations (3.2)-(3.4) can equivalently be written as two independent subsystems, one consisting of equations (A.55), (A.57) and (A.59) for the evolution of $\left\{y_{1, t}^{*}\right\}$, and the other consisting of (A.56), (A.58) and (A.60) for the evolution of $\left\{y_{2, t}^{*}\right\}$.

\footnotetext{
${ }^{31}$ As explained below, the "inessential" variables are not necessarily variables that are not needed for a description of the exact equilibrium dynamics implied by the model; they are redundant only for a description of the linearized dynamics.
} 
In the case that a decomposition of the form (4.6) with $q>0$ exists, the subsystem consisting of equations (A.56), (A.58) and (A.60) represents $q$ structural equations per period to determine the $q$ "inessential" variables. Thus there is no scope for adjustment of any of these $q$ variables as a policy decision, under the linearized dynamics. We state Assumption 5 only for the case in which $q>0$, since in the case that $q=0$, there are no inessential variables about which such an assumption needs to be stated. We can also neglect the case of a decomposition of the form (4.6) with $q=n$, as this would violate Assumption 2(a): the matrix (3.8) would be of at most rank $n<m$. We verify in Appendix $\mathrm{C}$ that Assumption 5 holds, for example, for the exact New Keynesian model presented in section 5 .

The following consequence of Assumption 5(b) is useful in what follows.

Lemma 13 Suppose that Assumption 2 holds so that a decomposition of the matrix pencil $\bar{I}-\mu \bar{A}$ of the form indicated by (4.6) exists, and suppose that 5(b) is satisfied. Then there exist non-singular matrices $N_{2}, R_{2}$, (of dimensions $\left.q \times q\right)$ such that

$$
N_{2}^{\prime}\left(J_{2}-\mu B_{2}\right) R_{2}^{\prime}=\left[\begin{array}{cc}
I-\mu G^{\prime} & 0 \\
0 & H^{\prime}-\mu I
\end{array}\right]
$$

where $G$ is of dimension $k_{2} \times k_{2}$ and $H$ is of dimension $\left(q-k_{2}\right) \times\left(q-k_{2}\right)$, all eigenvalues of the matrix $G$ have modulus less than 1 , and all eigenvalues of the matrix $H$ have modulus less than $\beta$.

The proof of this result is given in Appendix A.9.

In order to establish that the subsystem consisting of equations (A.56), (A.58) and (A.60) does indeed determine the $q$ endogenous variables $\left\{y_{2, t}^{*}\right\}$, one additional regularity condition is needed. Lemma 13 implies that the stable subspace of the system (A.56) is of the right dimension for determinacy; but we also need an assumption that guarantees consistency between the initial conditions (A.58) or (A.60) and the stable subspace of the system (A.56).

Assumption 6 Suppose that a decomposition of the matrix pencil $\bar{I}-\mu \bar{A}$ of the form indicated by equations (4.6) and (A.52) exists. Then the $(q \times q)$ matrix

$$
\left.\left[\begin{array}{cc}
X_{1}^{\prime} \\
0 & I_{q-k_{2}}
\end{array}\right] N_{2}^{\prime}\right]
$$

is invertible.

Note that this condition will be satisfied by a generic matrices satisfying our other assumptions; it would follow from (though is weaker than) an assumption that $J_{2}$ is of full rank. Given these assumptions, we can now show that the variables $\left\{y_{2 t}^{*}\right\}$ must be unaffected by policy.

Proposition 4 Let the linearized structural equations (3.2)-(3.4) satisfy Assumptions 2, 5, 6, and let the exogenous disturbances $\left\{\tilde{\xi}_{t}\right\}$ satisfy $\|\tilde{\xi}\|<\infty$. Then if $q>0$, there is a unique solution $\left\{y_{2 t}^{*}\right\}$ to the subsystem consisting of equations (A.56), (A.58) and (A.60) for all periods $t \geq t_{0}$ such that $\left\|y_{2}^{*}\right\|<\infty$. Hence in the case of any equilibrium $\left\{\tilde{y}_{t}\right\}$ consistent with (3.2)-(3.4) and such that $\|\tilde{y}\|<\infty$, the evolution of the "inessential" variables $\left\{y_{2 t}^{*}\right\}$ must be the same, independent of any policy choices.

The proof is in Appendix A.10. It follows that target criteria need be formulated only for the evolution of the "essential" variables $\left\{y_{1 t}^{*}\right\}$, and that in addressing the issues both of determinacy of equilibrium and of the optimality of the implied equilibrium, it suffices to consider the implications for the essential variables of the target criteria together with the structural equations (A.55), (A.57) and (A.59). 


\section{A.8.1 Essential vs. Inessential Target Variables}

While Assumption 2 and hence Lemma 3 allowed us to decompose the system of linearized FOCs (4.1) into (4.8) and (4.9), Assumption 5 allows us to decompose the system of equations (4.2) in a way conformable with our decomposition of (4.1).

Lemma 14 Suppose that Assumptions 2 and 5 are satisfied. Then equations (4.2) hold if and only if

$$
\begin{array}{r}
V_{2}\left[\hat{\varphi}_{1, t}-E_{t-1} \hat{\varphi}_{1, t}\right]=0 \\
W_{2}\left[\hat{\varphi}_{2, t}-E_{t-1} \hat{\varphi}_{2, t}\right]=0
\end{array}
$$

for all $t>t_{0}$.

The proof is in Appendix A.11.

In the case that our problem is to maximize the modified objective (2.9) defined by a vector of initial multipliers $\Theta_{t_{0}-1}$, we can also partition the initial conditions implied by the initial multipliers. The requirement that $\tilde{\varphi}_{2, t_{0}}=\tilde{\Theta}_{t_{0}-1}$ holds if and only if both sets of conditions

$$
\begin{aligned}
V_{2} \hat{\varphi}_{1, t_{0}} & =\Xi_{1, t_{0}-1} \\
W_{2} \hat{\varphi}_{2, t_{0}} & =\Xi_{2, t_{0}-1}
\end{aligned}
$$

hold, where we define

$$
\left[\begin{array}{l}
\Xi_{1, t} \\
\Xi_{2, t}
\end{array}\right]=\Xi_{t} \equiv\left(T_{2}^{-1}\right)^{\prime} \tilde{\Theta}_{t}
$$

Here $\Xi_{t_{0}-1}$ is an alternative representation of the initial multipliers $\Theta_{t_{0}-1}$, and $\Xi_{1, t_{0}-1}$ and $\Xi_{2, t_{0}-1}$ are respectively the first $\tilde{n}-k_{1}$ elements and the last $q-k_{2}$ elements of the vector $\Xi_{t_{0}-1}$. The multipliers $\Xi_{1, t}$ correspond to the $\tilde{n}-k_{1}$ forward-looking constraints among the structural equations governing the evolution of the $m-q$ "essential" variables $y_{1, t}^{*}$, while the multipliers $\Xi_{2, t}$ correspond to the $q-k_{2}$ forward-looking constraints among the structural equations that determine the $q$ "inessential" variables $y_{2, t}^{*}$.

Thus it is possible to decompose the complete system of first-order conditions (4.1)-(4.2) into two independent subsystems, one involving only the variables $\hat{\varphi}_{1, t}$ and the other involving only the variables $\hat{\varphi}_{2, t}$. The first subsystem consists of the conditions (4.8) that must hold for all $t \geq t_{0}$; conditions (A.62), that must hold for all $t>t_{0}$; and condition (A.64). The second subsystem consists of the conditions (4.9), that must hold for all $t \geq t_{0}$; conditions (A.63), that must hold for all $t>t_{0}$; and condition (A.65). As the following proposition states, the second subsystem of FOCs necessarily has a bounded solution (where again the norm is the one defined in (3.7)), regardless of the evolution of the target variables.

Proposition 5 Suppose that Assumptions 2, 5 and 6 are satisfied. Then there necessarily exists a process $\left\{\hat{\varphi}_{2, t}\right\}$ such that $\left\|\hat{\varphi}_{2}\right\|<\infty$ that satisfies conditions (4.9) for all $t \geq t_{0}$, (A.63) for all $t>t_{0}$, and the initial condition (A.65), regardless of the evolution of the target variables $\left\{\hat{\tau}_{2, t}\right\}$ (assuming only that $\left\|\hat{\tau}_{2}\right\|<\infty$, where the norms for both multipliers and target variables are defined as in (3.7)). Hence necessary and sufficient conditions for the existence of a process $\left\{\tilde{\varphi}_{t}\right\}$ such that $\|\tilde{\varphi}\|<\infty$ that satisfies conditions (4.1) for all $t \geq t_{0}$ and (4.2) for all $t>t_{0}$, consistent with given initial multipliers $\tilde{\Theta}_{t_{0}-1}$, are that the target variables $\left\{\hat{\tau}_{1, t}\right\}$ be such that there exists a process $\left\{\hat{\varphi}_{1, t}\right\}$ with $\left\|\hat{\varphi}_{1}\right\|<\infty$ that satisfies conditions (4.8) for all $t \geq t_{0}$, (A.62) for all $t>t_{0}$, and the initial condition (A.64). 
The proof is in Appendix A.12. Accordingly, our problem reduces to the design of a target criterion involving the variables $\left\{\hat{\tau}_{1, t}\right\}$ which is necessary and sufficient for the existence of a bounded process $\left\{\hat{\varphi}_{1, t}\right\}$ with the properties listed in the proposition.

\section{A.9 Proof of Lemma 13}

There is no vector $\hat{\varphi}_{2}(\mu) \neq 0$ such that $\hat{\varphi}_{2}(\mu)^{\prime}\left[J_{2}-\mu B_{2}\right]=0$ for all $\mu$. For if there did, the vector

$$
\varphi(\mu)=P^{-1}\left[\begin{array}{c}
0 \\
\hat{\varphi}_{2}(\mu)
\end{array}\right] \neq 0
$$

would satisfy (3.9), and this would violate Assumption 2(b). This implies that the pencil $J_{2}-\mu B_{2}$ must be of rank $q$, which (since it is a square pencil of dimension $q \times q$ ) implies that it is a regular pencil.

It follows from Theorem 3 of Gantmacher (1959, Chap. 12), or its version for a real canonical form proved in Appendix B.3, that $J_{2}-\mu B_{2}$ can be reduced to a strictly equivalent pencil of the form

$$
\left[\begin{array}{cc}
I_{l}-\mu \hat{G}^{\prime} & 0 \\
0 & \hat{H}^{\prime}-\mu I_{q-l}
\end{array}\right]
$$

where $\hat{G}^{\prime} \in \mathbb{R}^{l \times l}(0 \leq l \leq q)$ is a nilpotent matrix of the Jordan form (i.e., with ones on the first super diagonal and zeros everywhere else), and $\hat{H}^{\prime} \in \mathbb{R}^{(q-l) \times(q-l)}$ is a block-diagonal matrix of the real Jordan form. We can without loss of generality arrange the Jordan blocks of $\hat{H}^{\prime}$ as

$$
\hat{H}^{\prime}=\left[\begin{array}{cc}
\hat{H}_{11}^{\prime} & 0 \\
0 & H^{\prime}
\end{array}\right]
$$

where the invertible matrix $\hat{H}_{11}$ contains the eigenvalues with modulus greater than or equal to $\beta$, and $H$ contains only eigenvalues with modulus less than $\beta$. Premultiplying the pencil (A.66) by the invertible block-diagonal matrix

$$
\left[\begin{array}{ccc}
I_{l} & 0 & 0 \\
0 & \left(\hat{H}_{11}^{\prime}\right)^{-1} & 0 \\
0 & 0 & I_{q-k_{2}}
\end{array}\right]
$$

yields a strictly equivalent matrix pencil of the form (A.61), where all eigenvalues of

$$
G^{\prime}=\left[\begin{array}{cc}
\hat{G}^{\prime} & 0 \\
0 & \left(\hat{H}_{11}^{\prime}\right)^{-1}
\end{array}\right]
$$

have modulus less than or equal to $\beta^{-1}$ and all eigenvalues of $H$ have modulus less than $\beta$. It remains only to determine the dimensions of $G$ and $H$.

The existence of a decomposition of the form (A.61) implies that the factors of the characteristic polynomial $P(\lambda, \mu)$ defined in Assumption 5(b) are the same as those of

$$
\operatorname{det}\left[\lambda I-\mu G^{\prime}\right] \cdot \operatorname{det}\left[\lambda H^{\prime}-\mu I\right] .
$$

This implies the existence of a factorization of the form (A.53), where the $\left\{\gamma_{i}\right\}$ are the eigenvalues of $G$ and the $\left\{\eta_{j}\right\}$ are the eigenvalues of $H$. It follows that $G$ must be of dimension $k_{2} \times k_{2}$ and $H$ must be of dimension $\left(q-k_{2}\right) \times\left(q-k_{2}\right)$. Finally, since by Assumption 5(b), $\left|\gamma_{i}\right|<1$ for all $i$, all eigenvalues of $G$ must have modulus strictly smaller than 1 . 


\section{A.10 Proof of Proposition 4}

Premultiplying (A.56) by $N_{2}^{\prime}$ yields the pair of equations

$$
\begin{aligned}
E_{t} x_{1, t+1} & =G^{\prime} x_{1, t}+N_{21}^{\prime} \Gamma_{2} \tilde{\xi}_{t} \\
H^{\prime} E_{t} x_{2, t+1} & =x_{2, t}+N_{22}^{\prime} \Gamma_{2} \tilde{\xi}_{t}
\end{aligned}
$$

using the decomposition (A.61), defining

$$
x_{t} \equiv\left[\begin{array}{l}
x_{1 t} \\
x_{2 t}
\end{array}\right] \equiv\left(R_{2}^{-1}\right)^{\prime} y_{2 t}^{*},
$$

and partitioning $N_{2}^{\prime} \equiv\left[\begin{array}{l}N_{21}^{\prime} \\ N_{22}^{\prime}\end{array}\right]$ conformably with the partition of $x_{t}$.

Because all eigenvalues of $H^{\prime}$ have modulus less than $\beta$, and $\left\{\tilde{\xi}_{t}\right\}$ is bounded for all dates $t \geq t_{0}-1$, there is a unique process $\left\{x_{2 t}\right\}$ consistent with (A.68) and such that $\left\|x_{2}\right\|<\infty$, namely

$$
x_{2 t}=-\sum_{j=0}^{\infty}\left(H^{\prime}\right)^{j} N_{22}^{\prime} \Gamma_{2} E_{t} \tilde{\xi}_{t+j} .
$$

Then in any period $t$, given the past, current and expected future values of the exogenous disturbance process, and given the lagged expectations of $J_{2} E_{t-1} y_{2 t}^{*}$, equation (A.58) determines the value of $X_{1}^{\prime} J_{2} y_{2, t}^{*}$, while equation (A.70) determines the value of $x_{2 t}$ and hence of $H^{\prime} x_{2 t}$. We then have a system of equations of the form

$$
\left.\left[\begin{array}{cc}
X_{1}^{\prime} \\
0 & I_{q-k_{2}}
\end{array}\right] N_{2}^{\prime}\right] J_{2} y_{2, t}^{*}=\left[\begin{array}{c}
X_{1}^{\prime} J_{2} y_{2, t}^{*} \\
H^{\prime} x_{2 t}
\end{array}\right]
$$

to solve for $J_{2} y_{2, t}^{*}$, where all elements of the matrix on the right-hand side have been computed. Since the matrix on the left-hand side is invertible by Assumption 6, this system has a unique solution for $J_{2} y_{2, t}^{*}$. Using this solution, we can in turn solve for

$$
x_{1, t}=\left[\begin{array}{ll}
I & 0
\end{array}\right] N_{2}^{\prime} J_{2} y_{2, t}^{*} .
$$

Combining this solution for $x_{1 t}$ with (A.70), we have a unique solution for the entire vector $x_{t}$, given values of $J_{2} E_{t-1} y_{2, t}^{*}$ and the evolution of the exogenous disturbances. And given the value of $x_{t}$ in any period, equations (A.67) and (A.68) uniquely determine the values of $E_{t}\left[x_{1, t+1}\right]$ and $E_{t}\left[H^{\prime} x_{2, t+1}\right]$ respectively. This allows us to uniquely determine

$$
J_{2} E_{t} y_{2, t+1}^{*}=N_{2}^{-1}\left[\begin{array}{c}
E_{t} x_{1, t+1} \\
E_{t}\left[H^{\prime} x_{2, t+1}\right]
\end{array}\right] .
$$

Thus starting from given initial conditions $J_{2} E_{t_{0}-1} y_{2, t_{0}}^{*}$, we can uniquely solve for $x_{t_{0}}$, use this to uniquely solve for $J_{2} E_{t_{0}} y_{2, t_{0}+1}^{*}$, use this to uniquely solve for $x_{t_{0}+1}$, and so on recursively, eventually obtaining a unique solution for the entire process $\left\{x_{t}\right\}$, and hence a unique solution for the entire process $\left\{y_{2 t}^{*}\right\}$, using the relation $y_{2 t}^{*}=R_{2}^{\prime} x_{t}$.

This solution $\left\{y_{2 t}^{*}\right\}$ is the only solution such that $\left\|y_{2}^{*}\right\|<\infty$, if any solution exists. But one easily verifies that it is indeed such a solution. By construction, (A.58) is satisfied each period, and also both (A.67) and (A.68), which suffice to imply that (A.56) is satisfied each period. Moreover, the fact that all eigenvalues of $G$ have modulus less than 1 implies that the process $\left\{x_{t}\right\}$ constructed in this way satisfies $\|x\|<\infty$, so that the associated process $\left\{y_{2 t}^{*}\right\}$ satisfies $\left\|y_{2}^{*}\right\|<\infty$. Hence all conditions for a solution are satisfied. 


\section{A.11 Proof of Lemma 14}

Suppose that (4.2) holds for any period $t>t_{0}$, and suppose that Assumption 2 holds. Lemma 3 then implies that there exist matrices $P, Q$ that define the decomposition (4.6), and Assumption 5 allows us to decompose conditions (4.2) into separate subsystems as well. It follows from (A.52) that

$$
\begin{aligned}
{\left[\begin{array}{l}
\hat{\varphi}_{1, t} \\
\hat{\varphi}_{2, t}
\end{array}\right] } & =P\left[\begin{array}{cc}
T_{1}^{\prime} & 0 \\
0 & T_{2}^{\prime}
\end{array}\right]\left[\begin{array}{cc}
\left(T_{1}^{-1}\right)^{\prime} & 0 \\
0 & \left(T_{2}^{-1}\right)^{\prime}
\end{array}\right]\left[\begin{array}{l}
\tilde{\varphi}_{1, t} \\
\tilde{\varphi}_{2, t}
\end{array}\right] \\
& =\left[\begin{array}{cccc}
U_{1} & 0 & U_{2} & 0 \\
0 & X_{1} & 0 & X_{2}
\end{array}\right]\left[\begin{array}{l}
\left(T_{1}^{-1}\right)^{\prime} \tilde{\varphi}_{1, t} \\
\left(T_{2}^{-1}\right)^{\prime} \tilde{\varphi}_{2, t}
\end{array}\right]
\end{aligned}
$$

so that

$$
\begin{aligned}
\hat{\varphi}_{1, t} & =\left[\begin{array}{ll}
U_{1} & 0
\end{array}\right]\left(T_{1}^{-1}\right)^{\prime} \tilde{\varphi}_{1, t}+\left[\begin{array}{cc}
U_{2} & 0
\end{array}\right]\left(T_{2}^{-1}\right)^{\prime} \tilde{\varphi}_{2, t} \\
\hat{\varphi}_{2, t} & =\left[\begin{array}{ll}
0 & X_{1}
\end{array}\right]\left(T_{1}^{-1}\right)^{\prime} \tilde{\varphi}_{1, t}+\left[\begin{array}{ll}
0 & X_{2}
\end{array}\right]\left(T_{2}^{-1}\right)^{\prime} \tilde{\varphi}_{2, t} .
\end{aligned}
$$

Equations (A.71) and (A.72) respectively imply that

$$
\begin{aligned}
\hat{\varphi}_{1, t}-E_{t-1} \hat{\varphi}_{1, t} & =\left[\begin{array}{ll}
U_{1} & 0
\end{array}\right]\left(T_{1}^{-1}\right)^{\prime}\left(\tilde{\varphi}_{1, t}-E_{t-1} \tilde{\varphi}_{1, t}\right) \\
\hat{\varphi}_{2, t}-E_{t-1} \hat{\varphi}_{2, t} & =\left[\begin{array}{ll}
0 & X_{1}
\end{array}\right]\left(T_{1}^{-1}\right)^{\prime}\left(\tilde{\varphi}_{1, t}-E_{t-1} \tilde{\varphi}_{1, t}\right) .
\end{aligned}
$$

Recall from Assumption 5 that both $\left[\begin{array}{ll}U_{1} & U_{2}\end{array}\right]$ and $\left[\begin{array}{cc}X_{1} & X_{2}\end{array}\right]$ are invertible matrices, and define the matrices $V_{1}, V_{2}, W_{1}$, and $W_{2}$ as in (A.54). Note that it follows from these definitions that

$$
\begin{aligned}
V_{1} U_{1}=I_{k_{1}}, & V_{1} U_{2}=0, \quad V_{2} U_{1}=0, \quad V_{2} U_{2}=I_{\tilde{n}-k_{1}} \\
W_{1} X_{1}=I_{k_{2}}, & W_{1} X_{2}=0, \quad W_{2} X_{1}=0, \quad W_{2} X_{2}=I_{q-k_{2}} .
\end{aligned}
$$

Premultiplying (A.73) by $V_{2}$ yields (A.62); premultiplying (A.74) by $W_{2}$ yields (A.63). Hence (A.62)-(A.63) must hold for all $t>t_{0}$.

Conversely, suppose that (A.62)-(A.63) hold in some period $t>t_{0}$. Using (A.71), (A.62) implies that

$$
\left[\begin{array}{ll}
I & 0
\end{array}\right]\left(T_{2}^{-1}\right)^{\prime}\left(\tilde{\varphi}_{2, t}-E_{t-1} \tilde{\varphi}_{2, t}\right)=0 .
$$

Similarly, using (A.72), (A.63) implies that

$$
\left[\begin{array}{ll}
0 & I
\end{array}\right]\left(T_{2}^{-1}\right)^{\prime}\left(\tilde{\varphi}_{2, t}-E_{t-1} \tilde{\varphi}_{2, t}\right)=0 .
$$

Together, these conditions imply that

$$
\left(T_{2}^{-1}\right)^{\prime}\left(\tilde{\varphi}_{2, t}-E_{t-1} \tilde{\varphi}_{2, t}\right)=0
$$

which implies (4.2). Hence (4.2) must hold for all $t>t_{0}$.

\section{A.12 Proof of Proposition 5}

In order to prove Proposition 5, we make use of the following preliminary result. 
Lemma 15 Suppose that there exist matrices $P, Q$ that define a decomposition of the form (4.6), and that Assumptions 2,5 and 6 hold. Then the $q \times q$ matrix

$$
\left.\left[\begin{array}{cc}
I_{k_{2}} & 0
\end{array}\right] R_{2} J_{2}^{\prime}\right]
$$

is invertible.

Proof. Let $x$ be an arbitrary vector of length $q$, partitioned as in (A.69). By Assumption 6, knowing the values of the vectors $X_{1}^{\prime} J_{2} R_{2}^{\prime} x$ and $[0 I] N_{2}^{\prime} J_{2} R_{2}^{\prime} x$ allows one to reconstruct the entire vector $J_{2} R_{2}^{\prime} x$, and hence all elements of

$$
N_{2}^{\prime} J_{2} R_{2}^{\prime} x=\left[\begin{array}{c}
x_{1} \\
H^{\prime} x_{2}
\end{array}\right]
$$

Since the elements of $[0 I] N_{2}^{\prime} J_{2} R_{2}^{\prime} x=H^{\prime} x_{2}$ provide information only about the elements of $x_{2}$, it must be that each of the $k_{2}$ independent directions of variation of the elements of $x_{1}$ affects the elements of $X_{1}^{\prime} J_{2} R_{2}^{\prime} x$ in an independent direction. Thus Assumption 6 implies that the $k_{2} \times k_{2}$ matrix

$$
\Xi \equiv X_{1}^{\prime} J_{2} R_{2}^{\prime}\left[\begin{array}{c}
I_{k_{2}} \\
0
\end{array}\right]
$$

is invertible.

Next, let $\hat{\varphi}$ be another arbitrary vector of length $q$, and let

$$
\check{\varphi} \equiv N_{2}^{-1} \hat{\varphi} \equiv\left[\begin{array}{c}
\check{\varphi}_{1} \\
\check{\varphi}_{2}
\end{array}\right]
$$

One observes that

$$
R_{2} J_{2}^{\prime} \hat{\varphi}=\left[R_{2} J_{2}^{\prime} N_{2}\right] \check{\varphi}=\left[\begin{array}{cc}
I & 0 \\
0 & H
\end{array}\right] \check{\varphi}=\left[\begin{array}{c}
\check{\varphi}_{1} \\
H \check{\varphi}_{2}
\end{array}\right]
$$

Then in the case that

$$
\hat{\varphi}=X_{1} f
$$

for some vector $f$ of length $k_{2}$, it follows that $\check{\varphi}_{1}=\Xi^{\prime} f$, where $\Xi$ is defined in (A.78).

Because $\Xi$ (and hence $\Xi^{\prime}$ ) is invertible, if for any $\hat{\varphi}$ of the form (A.79), $\check{\varphi}_{1}=0$, it follows that $f=0$ and hence that $\hat{\varphi}=0$. But it follows from (A.76) that a vector $\hat{\varphi}$ has a representation of the form (A.79) if and only if $W_{2} \hat{\varphi}=0$. Hence if any vector $\hat{\varphi}$ satisfies both $W_{2} \hat{\varphi}=0$ and $\check{\varphi}_{1}=0$, it must satisfy $\hat{\varphi}=0$. Alternatively, if it satisfies both the linear restrictions $W_{2} \hat{\varphi}=0$ and $\left[\begin{array}{ll}I & 0\end{array} R_{2} J_{2}^{\prime} \hat{\varphi}=0\right.$, it must be a zero vector. It follows from this that the matrix (A.77) must be invertible.

We may now proceed with the proof of Proposition 5. Let $\left\{\hat{\tau}_{2, t}\right\}$ be any process satisfying $\left\|\hat{\tau}_{2}\right\|<\infty$. Given Assumption 2, Lemma 3 implies that a decomposition of the form (4.6) exists. With Assumption 5 furthermore satisfied, Lemma 13 guarantees in turn that there exist nonsingular matrices $N_{2}, R_{2}$ such that (A.61) holds. Then premultiplying (4.9) by $R_{2}$ and using (A.61) yields

$$
\begin{aligned}
\check{\varphi}_{1 t} & =\beta G E_{t} \check{\varphi}_{1, t+1}-\beta \check{\tau}_{1 t}, \\
E_{t} \check{\varphi}_{2, t+1} & =\left(\beta^{-1} H\right) \check{\varphi}_{2, t}+\check{\tau}_{2 t},
\end{aligned}
$$

where

$$
\check{\varphi}_{t} \equiv\left[\begin{array}{c}
\check{\varphi}_{1 t} \\
\check{\varphi}_{2 t}
\end{array}\right] \equiv N_{2}^{-1} \hat{\varphi}_{2 t}, \quad\left[\begin{array}{c}
\check{\tau}_{1 t} \\
\check{\tau}_{2 t}
\end{array}\right] \equiv R_{2} \hat{\tau}_{2 t}
$$


Because all eigenvalues of $\beta G$ have modulus less than 1, (A.80) has a solution for $\left\{\check{\varphi}_{1 t}\right\}$ such that $\left\|\check{\varphi}_{1}\right\|<\infty$, given by

$$
\check{\varphi}_{1 t}=-\beta \sum_{j=0}^{\infty}(\beta G)^{j} E_{t} \check{\tau}_{1, t+j} .
$$

Then in any period $t$, the value of

$$
\check{\varphi}_{1 t}=\left[\begin{array}{ll}
I & 0
\end{array}\right] R_{2} J_{2}^{\prime} \hat{\varphi}_{2 t}
$$

is given by (A.82), while the value of $W_{2} \hat{\varphi}_{2 t}$ is given by (A.63). Thus the values of both $\check{\varphi}_{1 t}$ and $W_{2} \hat{\varphi}_{2 t}$ are given as functions of variables that are exogenous and/or predetermined in period $t$. But Lemma 15 implies that the mapping from the linear space of vectors $\hat{\varphi}$ to the values of $\check{\varphi}_{1}$ and $W_{2} \hat{\varphi}$ is an isomorphism, so this system of equations can be uniquely solved for the value of $\hat{\varphi}_{2 t}$. We thus obtain a unique solution for $\hat{\varphi}_{2 t}$ as a linear function of $E_{t-1} \hat{\varphi}_{2 t}$ and the $E_{t} \check{\tau}_{1, t+j}$ for $j \geq 0$.

This solution for $\hat{\varphi}_{2 t}$ allows us to solve for $\check{\varphi}_{2 t}$, and substituting this into (A.81) yields a value for $E_{t} \check{\varphi}_{2, t+1}$ as a linear function of $E_{t-1} \hat{\varphi}_{2 t}, \check{\tau}_{2 t}$, and the $E_{t} \check{\tau}_{1, t+j}$ for $j \geq 0$. The solution (A.82) implies that

$$
E_{t} \check{\varphi}_{1, t+1}=-\beta \sum_{j=0}^{\infty}(\beta G)^{j} E_{t} \check{\tau}_{1, t+j+1} .
$$

Hence we can solve for the complete vector $E_{t} \check{\varphi}_{t+1}$ as a linear function of $E_{t-1} \hat{\varphi}_{2 t}$ and the exogenous state. Alternatively, we can solve for $E_{t} \hat{\varphi}_{2, t+1}=N_{2} E_{t} \check{\varphi}_{t+1}$ as a linear function of $E_{t-1} \hat{\varphi}_{2 t}$ and the exogenous state. Thus starting from an initial condition $E_{t_{0}-1} \hat{\varphi}_{2 t_{0}}$, we can solve for $\hat{\varphi}_{2 t_{0}}$ and $E_{t_{0}} \hat{\varphi}_{2, t_{0}+1}$; using this solution we can solve for $\hat{\varphi}_{2, t_{0}+1}$ and $E_{t_{0}+1} \hat{\varphi}_{2, t_{0}+2}$; and so on recursively.

Thus it is possible to solve for the complete evolution $\left\{\hat{\varphi}_{2 t}\right\}$ for all $t \geq t_{0}$, given the initial condition $E_{t_{0}-1} \hat{\varphi}_{2 t_{0}}$ and the evolution of the exogenous state. By construction (A.80) and (A.81) are satisfied for each $t \geq t_{0}$, which implies that (4.9) is satisfied for each $t \geq t_{0}$. Likewise, (A.63) is satisfied for each $t>t_{0}$ by construction. Thus we obtain a process $\left\{\hat{\varphi}_{2 t}\right\}$ that satisfies both (4.9) for all $t \geq t_{0}$ and (A.63) for all $t>t_{0}$. Moreover, because all eigenvalues of $\beta^{-1} H$ have modulus less than 1, (A.81) implies that the constructed solution satisfies $\left\|\hat{\varphi}_{2}\right\|<\infty$. 